\title{
27. GEOCHEMICAL SECTION OF THE TAG HYDROTHERMAL MOUND ${ }^{1}$
}

\author{
Yves Fouquet, ${ }^{2}$ Katell Henry, ${ }^{2}$ Richard Knott, ${ }^{3}$ and Pierre Cambon ${ }^{2}$
}

\begin{abstract}
Drilling operations to 125 mbsf in the TAG mound have clarified the geochemical organization of the mound and stockwork zone. Bulk chemical analyses show that base metal values are very low within the mound and underlying stockwork zone. Low values may be explained by a combination of two processes. (1) During maturation, metals are leached from primary sulfides equivalent to those formed in chimneys at the surface. (2) Sulfide formed in situ as basalt replacements and veins are not enriched in trace metals. Another possibility is that the base metal-rich zones, particularly the copper-rich zone, were not drilled. The $\mathrm{Zn}$-rich zone probably occurs only in the surface chimneys. A Cu-rich zone may be present as a vertical zone below the Black Smoker Complex but was not drilled. TAG has a geochemical and mineralogical asymmetry that may be explained by less active leaching and maturation processes on the western side of the mound. This part was probably formed as talus of different types of material related to a north-south fault feeding the black smoker. Leaching is more efficient on the eastern side, and all metals are removed from the surface sulfide at TAG-1. At TAG-2, metals are only concentrated within the first $10 \mathrm{~m}$. Thus, the source of $\mathrm{Zn}$ in the white smoker fluid has not been identified. The abundance of quartz within the mound indicates a high temperature of formation under stable physico-chemical conditions. Trace metals associated with $\mathrm{Zn}$ are $\mathrm{Cd}, \mathrm{Zn}, \mathrm{Ag}, \mathrm{Sb}$, and $\mathrm{Pb}$. Principal component analyses (PCA) studies show that these metals occur within sphalerite or within low-temperature pyrite associated with sphalerite. Cobalt and $\mathrm{Se}$ are not always specifically correlated with $\mathrm{Cu}$, but they occur preferentially in high-temperature pyrite formed under stable conditions. In relation to these different observations we consider that the mound growth is caused by three different processes. (1) At the surface the sulfides formed within the chimneys are progressively buried and recrystallized, contributing to the surface growth of the massive sulfide mound. This material can be relatively enriched in $\mathrm{Zn}$, which is later leached through a zone refining process. (2) In the deepest part the basalt is progressively silicified and replaced by a pyrite/quartz assemblage contributing to the internal growth and stockwork formation. These samples correspond to high-temperature pyrite and were never enriched in $\mathrm{Zn}$ and associated trace metals. Thus, most of the mound is depleted in trace metals. (3) The third process is the anhydrite veining that also contributes to the internal growth. Most of the anhydrite should be dissolved when the activity ceases. However, veins are often associated with a chalcopyrite selvage that contributes to the inner mound growth.
\end{abstract}

\section{INTRODUCTION}

Despite all the studies on oceanic hydrothermal systems, the mechanisms of sulfide precipitation, maturation, zone refining, and chemical zonation within a mound are very poorly documented. Ocean Drilling Program (ODP) Leg 158 was designed to study the subsurface nature of an active hydrothermal system on a slowspreading, sediment-free mid-ocean ridge. The site chosen for this study was the active Trans-Atlantic Geotraverse (TAG) hydrothermal mound $\left(26^{\circ} 08^{\prime} \mathrm{N}\right.$ on the Mid-Atlantic Ridge; Rona et al., 1986). According to the preliminary drilling results the mound was estimated to be 4 million metric tons (Humphris et al., 1995), making it equivalent in size to some ore deposits on land, such as those in Cyprus, Oman, and other ophiolites (Alabaster et al., 1980; Constantinou and Govett, 1973; Ixer et al., 1984).

In this paper, we present chemical analyses for major and trace elements obtained on sulfide samples from 5 drilling sites at the TAG mound (Fig. 1). Statistical analyses are used to discuss the behavior of elements during the mineral formation and maturation of the mound and to compare the geochemistry of TAG with two other major mature oceanic deposits in basaltic environments. Detailed mineralogical studies for the samples presented here will be found in Knott et al. (Chap. 1, this volume).

${ }^{1}$ Herzig, P.M., Humphris, S.E., Miller, D.J., and Zierenberg, R.A. (Eds.), 1998 Proc. ODP, Sci. Results, 158: College Station, TX (Ocean Drilling Program).

${ }^{2}$ IFREMER, Centre de Brest, B.P. 70, 29280 Plouzané, France. fouquet@ifremer.fr France.

\section{TAG GEOLOGICAL SETTING AND PREVIOUS STUDIES}

\author{
Regional Setting
}

The main characteristics of the TAG field have been summarized recently (Rona et al., 1993b; Shipboard Scientific Party, 1996). The TAG volcanic segment has the morphology of an hour glass (Purdy et al., 1990; Sempéré et al., 1990; Smith and Cann, 1992), narrowing and shallowing toward the center of the segment. At about $26^{\circ} 10^{\prime} \mathrm{N}$, approximately the middle of the TAG segment, the east wall forms a broad indent that reduces the width of the valley floor from about 9 to $6 \mathrm{~km}$. Here, through a series of steps formed by fault blocks (Temple et al., 1979), the wall rises from the valley floor (near $4000 \mathrm{~m}$ depth) to a height of $2000 \mathrm{~m}$. The TAG hydrothermal field is located at the eastern base of this indent and extends over an area of at least $5 \times 5 \mathrm{~km}$ along the eastern median valley wall. It consists of presently active low- and high-temperature zones, as well as a number of fossil inactive deposits. The zone of low-temperature activity occurs between 2400 and $3100 \mathrm{~m}$ depth on the east wall and includes massive, layered deposits of Mn-oxide (birnessite), Fe-oxide (amorphous), and Fe-silicate (nontronite) (Rona et al., 1984; Thompson et al., 1985). The presently active black smoker system occurs at the junction between the rift valley floor and the east wall at approximately $26^{\circ} 08^{\prime} \mathrm{N}, 44^{\circ} 49^{\prime} \mathrm{W}$, at a water depth of about $3620-3700 \mathrm{~m}$, and lies on basaltic oceanic crust of at least 100 ka (Rona et al., 1993a, 1993b). 
Figure 1. Detailed bathymetry of the active TAG mound. The locations of drill holes A through $P$ are shown (Shipboard Scientific Party, 1996). Dashed lines = possible faults.

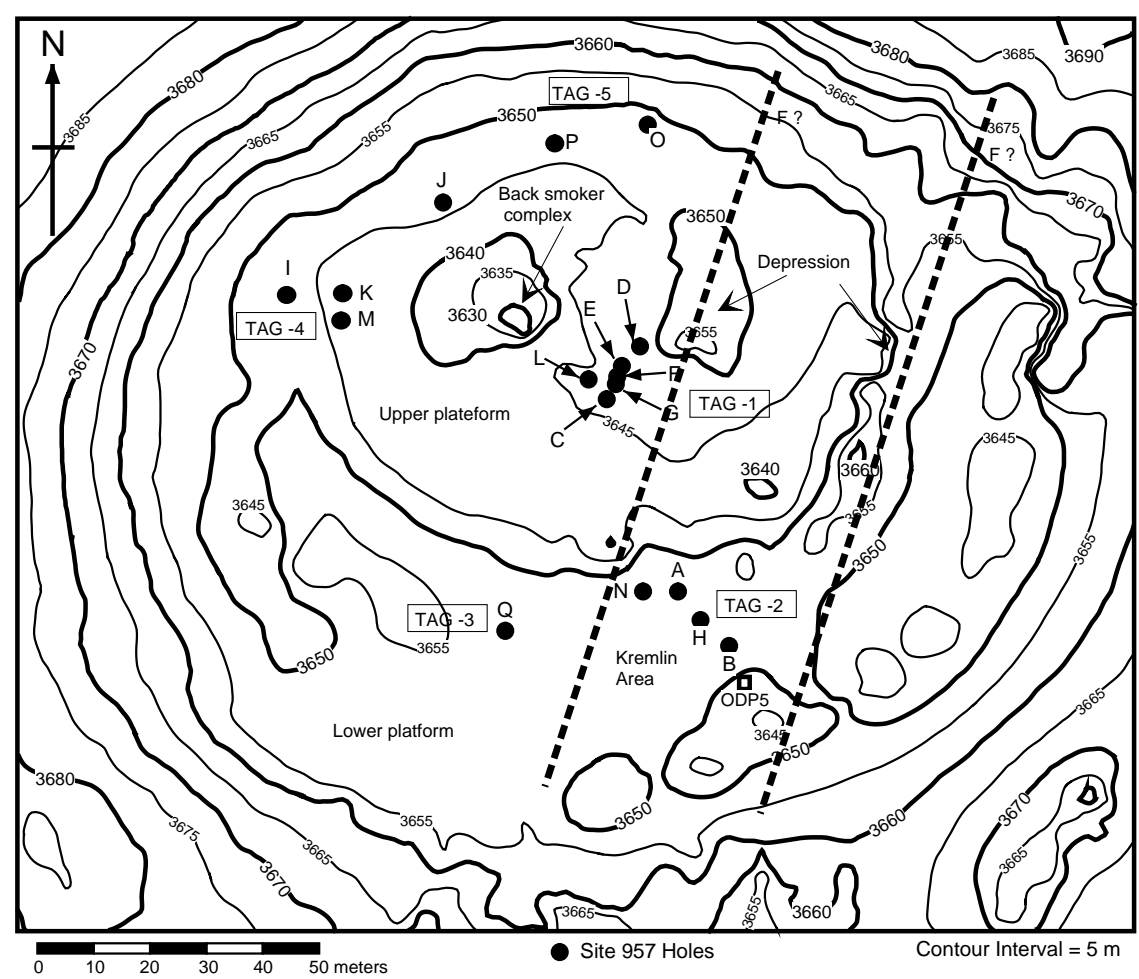

\section{The TAG Mound}

The TAG mound is circular and measures $200 \mathrm{~m}$ in diameter and $50 \mathrm{~m}$ in height (Fig. 1). It exhibits two distinct platforms at water depths of about 3650 and $3644 \mathrm{~m}$ (Fig. 2), which may represent two phases of active growth (Humphris et al., 1994). The mound is surrounded by an apron dominated by carbonate and metalliferous sulfide-oxide sediment and is composed of massive sulfides and anhydrite. Distinct sample types are distributed from the central to the outer areas of the mound surface (Rona et al., 1993a; Tivey et al., 1995). A cluster of chalcopyrite-anhydrite-rich black smoker chimneys (Black Smoker Complex) emitting fluids of up to $363^{\circ} \mathrm{C}$ is located northwest of the mound's center. This chimney cluster sits on top of a 10- to 15-m-high, 20- to 30-m-diameter cone, the surface of which is covered by a 3- to 6-cm-thick, platelike layer of massive chalcopyrite and marcasite, with interspersed corroded blocks of massive anhydrite containing variable amounts of chalcopyrite and pyrite. The tops of both the upper and lower platforms of the mound are relatively flat with irregular surfaces. Samples of amorphous Fe-oxyhydroxide and silica have been recovered from the west, south, and east rims of the mound. Bulbous, mixed $\mathrm{Zn}, \mathrm{Fe}$, and $\mathrm{Cu}-\mathrm{Fe}$ sulfides with cavities filled by amorphous silica were recovered from the northern rim and central portions of the mound (Tivey et al., 1995). A complex of white smokers venting fluids from $260^{\circ}$ to $300^{\circ} \mathrm{C}$ is located in the southeast quadrant of the mound approximately $70 \mathrm{~m}$ away from the Black Smoker Complex. These Kremlin-like spires are small (1-2 m) and are comprised predominantly of low-Fe sphalerite with minor amounts of chalcopyrite, pyrite, and amorphous silica. Fluids from the white smokers have a very low $\mathrm{pH}\left(3\right.$ at $\left.23^{\circ} \mathrm{C}\right)$ and less $\mathrm{Fe}$ than the black smoker fluids. In addition, they are depleted in $\mathrm{Cu}$ and enriched in $\mathrm{Zn}$ relative to the black smoker. The $\mathrm{Cu}$ content is respectively $135 \mu \mathrm{mol} / \mathrm{L}$ in the black smoker and only $3 \mu \mathrm{mol} / \mathrm{L}$ in the white smoker; $\mathrm{Zn}$ is $46 \mu \mathrm{mol} / \mathrm{L}$ in the black smoker and up to $400 \mu \mathrm{mol} / \mathrm{L}$ in the white smoker (Edmond et al., 1995). The white smoker fluids are thought to be derived from the more focused black smoker fluids by a combination of conductive cooling and mixing with seawater and precipitation of sulfides within the mound (Edmond et al., 1995; Tivey et al., 1995). Mineralogy and chemistry of surface deposits have been described in several papers (Thompson et al., 1985, 1988, Tivey et al., 1995). Several specific studies were made on the concentration of gold at the surface of the deposit (Hannington et al., 1995, 1988; Herzig et al., 1991).

Mass wasting of the edges of the mound results in steep outer slopes to the west, north, and east. Two sample types are exposed: (1) pyrite-rich blocks with trace amounts of late-stage amorphous silica, quartz, and goethite and with outer oxidized layers that include atacamite; and (2) deep red to orange-brown blocks of amorphous Feoxyhydroxide, goethite, hematite, amorphous silica, and quartz.

Fluids exiting the Black Smoker Complex are extremely focused. Fluids emanating from the Kremlin area have undergone conductive cooling and mixing with seawater, as evidenced by the presence of amorphous silica and by the chemistry of the fluids (Edmond et al., 1995). As the fluid cools and circulates within the mound, pyrite is precipitated.

Geochronological studies suggest that the mound is $\sim 40-50 \mathrm{ka}$ (Lalou et al., 1990). Activity has been intermittent over the past 20 k.y., with a periodicity of 5-6 k.y. (Lalou et al., 1993). The present activity began about $50 \mathrm{yr}$ ago after a hiatus of about 5 k.y. (Lalou et al., 1993).

\section{Principal Drilling Results}

This description summarizing the principal characteristics of each group of holes is taken from (Shipboard Scientific Party, 1996). Seventeen holes were drilled at five locations on the active TAG hydrothermal mound (Figs. 1, 2). Six holes (of which four produced core) were drilled just to the east of the Black Smoker Complex (TAG-1 area) on the upper terrace, and produced a section down to $125 \mathrm{mbsf}$ through the hydrothermal mound and into the upper part of the stockwork zone. Four holes were drilled in the Kremlin area (TAG-2 area) on the lower terrace; these holes resulted in maximum penetration of 54.3 mbsf. A single hole was drilled at a second location (TAG-3 area) on the lower terrace south of the Black Smoker Complex and west of the TAG-2 area, but only drill cuttings were retrieved down to 14.5 mbsf. Four holes were drilled on the upper terrace on the western side of the mound (TAG-4) to recover a section through the 


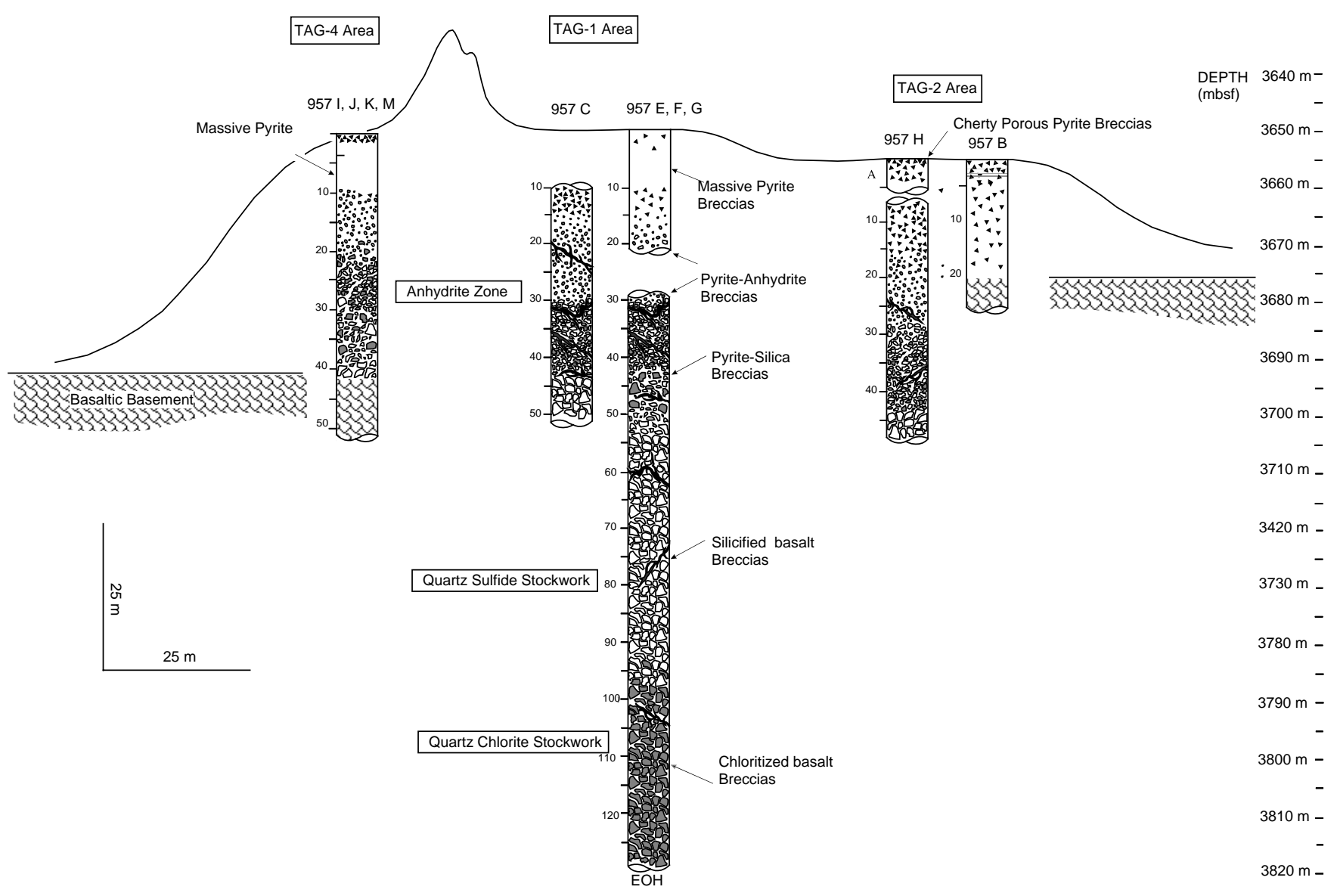

Figure 2. Schematic lithologic stratigraphy of the TAG hydrothermal mound from rock recovered from TAG-1, TAG-2, and TAG-4 (Shipboard Scientific Party, 1996).

sulfides and into the stockwork zone in an area of low conductive heat flow (Becker et al., 1993) and to determine the extent of the sulfides and stockwork in this area. Core was recovered down to 51.2 mbsf. Finally, two holes were drilled on the northern side of the upper terrace (TAG-5 area) to determine the lateral heterogeneity of the sulfide mineralization and to delineate the northern extent of the underlying stockwork zone. Coring here penetrated to 59.4 mbsf.

Figure 2 summarizes the stratigraphy of the mound (Shipboard Scientific Party, 1996). Breccias of various types dominate the sulfide mound and the upper part of the stockwork. Different rock types-massive pyrite breccia, pyrite-anhydrite breccia, and pyritesilica-anhydrite breccia-are distinguished on the basis of relative mineral abundances. They display different degrees of brecciation, cementation and replacement of pre-existing sulfides.

The existence of a normal fault or series of fault steps beneath the mound is suggested in part by about $15-20 \mathrm{~m}$ of displacement of basalt basement on opposite sides of the mound and by the presence of several major lineaments to the north and east of the mound, although these have not been confirmed by observation. A series of scarps aligned in a roughly north-south orientation may account for much of the surface morphology of the mound. These scarps are roughly parallel to the bathymetric lineaments observed off the flanks of the deposit (Kleinrock et al., 1996).

Four major zones can be distinguished. Clast-supported massive pyrite and pyrite breccias with sulfide cement dominate the upper $10-20 \mathrm{~m}$ of the mound at every location. This is followed by an anhydrite-rich zone that comprises matrix-supported, pyrite-anhydrite breccias and pyrite-anhydrite-silica breccias in the TAG-1 area. At the scale of the cores sulfide clasts are centimeter-sized and often have a round shape (see Knott et al., Chap. 1, this volume, for details on mineralogy and petrology). The TAG-5 area, however, contains only vein-related, pyrite-anhydrite breccias. In the TAG- 2 area, anhydrite is present only as thin veins and vug fillings; in the TAG-4 area, anhydrite is virtually absent. However, recovery at the TAG-2 and TAG-4 areas was so low that the possibility exists that anhydrite was preferentially not recovered. With increasing depth, the amount of quartz-pyrite mineralization and quartz veining increases and represents the top of the quartz-sulfide stockwork zone, which typically includes pyrite-silica breccias overlying silicified basalt breccias. This zone was observed at all holes that penetrated $>40$ mbsf, suggesting that the thickness of the mound is comparable to its relief on the seafloor. A quartz-chlorite stockwork zone was sampled at depths greater than 100 mbsf in Hole 957E (TAG-1).

A very hard layer containing red and gray chert was encountered in the upper few meters of the cores recovered from the TAG-2, TAG-3, and TAG- 4 areas. This most likely results from the precipitation of silica from hydrothermal fluids percolating through the mound and forming a silica cap and may be analogous to the silica caps overlying many volcanogenic massive sulfide deposits on land (Franklin et al., 1981).

\section{TAG-1 Area (East Side of the Black Smoker Complex)}

The upper few meters of the core contain fragments of massive granular pyrite and chalcopyrite, which likely represent near-surface hydrothermal precipitates derived from sulfide crusts and chimney talus. From the surface down to $15 \mathrm{mbsf}$, the core consists dominantly of porous to granular pyrite and pyrite breccias. Below $15 \mathrm{mbsf}$, an 
anhydrite-rich zone is present that consists of pyrite-anhydrite and pyrite-silica-anhydrite breccias that extend down to 45 mbsf. Abundant quartz-pyrite mineralization and quartz veining immediately beneath the anhydrite-rich zone represent the top of the stockwork zone, suggesting that the thickness of the mound in this area is about $30 \mathrm{~m}$. Between about 45 and 100 mbsf, a quartz-rich zone is composed mainly of pyrite-silica breccias, and of silicified basalt breccias at its deepest part. Below about $100 \mathrm{mbsf}$, the quartz-rich zone grades into a chloritic stockwork where chloritized and weakly mineralized basalt is the dominant lithology. Anhydrite veining is abundant throughout the vertical extent of the section, but it is best developed in the pyrite-anhydrite and pyrite-silica breccias (15-45 mbsf), where veins up to $45 \mathrm{~cm}$ in width are present. These veins comprise complex, multistage fracture fillings and cavity linings, some of which include disseminated, fine-grained pyrite and chalcopyrite, and trace amounts of hematite. The occurrence and size of veins decrease downward and correlate with an increase in the amount of quartz.

\section{TAG-2 Area ("Kremlin" White Smoker)}

Hydrothermal precipitates recovered from the topmost section at the TAG-2 Kremlin area include red-brown, sulfide-rich sand and mud (most likely drill cuttings) with abundant chert clasts and a few small pieces of sphalerite, pyrite, and chalcopyrite. A hard layer in the top few meters of each hole at TAG-2 consists of mixed pyrite and chert clasts in a dominantly cherty matrix. Clasts of similar red and gray chert are also common in the underlying massive, porous pyrite to a depth of about $10 \mathrm{mbsf}$. Most of the massive sulfide material recovered from the TAG-2 area occurs in the upper $20 \mathrm{~m}$ of the section as massive porous pyrite and porous, nodular pyrite breccias in a sandy pyrite matrix. The breccias are dominantly matrix-supported and contain only minor anhydrite cement. The TAG-2 area does not contain as wide a variety of breccias as at the TAG- 1 or TAG-4 sites, suggesting that the TAG-2 area has experienced a different history of brecciation, cementation, and veining than similar rocks drilled in the other areas of the mound.

Between 20 and $30 \mathrm{mbsf}$, the massive pyrite breccias grade into pyrite-silica breccias with anhydrite veining. Silicified basalt fragments and hyaloclastite first occur at about $30 \mathrm{mbsf}$, and the basalt fragments become increasingly abundant with depth. Deeper in the section, the fragments in the pyrite-silica breccias become larger and more angular and are interspersed with sections of brecciated and silicified basalt. At about $40 \mathrm{mbsf}$, the pyrite-silica breccias grade into more massive, silicified basalt breccias, and chloritized basalt fragments are present locally. These breccias appear to represent the upper part of the stockwork zone.

At the southeastern edge of the TAG-2 area, a section of chloritized pillow-rim breccia with a matrix of chlorite, quartz, and hematite was recovered at the base of the massive sulfides, which overlies partly altered basalt. This is interpreted to be the uppermost basement or a portion of a basaltic flow. The presence of chloritized pillow-rim breccia at the contact between relatively fresh basalt and the base of the massive sulfides indicates less pervasive hydrothermal flow along the margins of the mound than at its central part.

\section{TAG-3 Area (South Side of the Black Smoker Complex)}

Fine- to medium-grained drill cuttings consisting of silt- and sand-sized grains and fragments of massive pyrite, red and gray chert, partially silicified $\mathrm{Fe}$-oxides, and trace amounts of chalcopyrite were recovered.

\section{TAG-4 Area (West Side of the Black Smoker Complex)}

Core recovered from four holes in the TAG- 4 area indicates that this part of the mound consists mainly of massive sulfide crusts and sulfide-cemented breccias. In addition, significantly higher amounts of sphalerite, marcasite, and amorphous silica are present in these samples than in samples drilled elsewhere on the mound. Anhydrite is virtually absent in the TAG- 4 cores.

In this area, the upper $10 \mathrm{~m}$ of the mound consist of porous colloform pyrite + marcasite with local red and gray chert, and minor chalcopyrite, below which there is a 10-m-thick zone of massive pyrite and massive pyrite breccia with minor sphalerite and a few altered basalt clasts. Between 20 and 30 mbsf, massive pyrite grades downward into pyrite-silica breccia with abundant silicified basalt fragments up to $10 \mathrm{~cm}$ in size. Silicified basalt breccias are the dominant lithology between 30 and $42 \mathrm{mbsf}$, followed by an abrupt transition into slightly to moderately altered basalt having chloritized halos down to 51.2 mbsf.

The breccias consist of angular to subrounded clasts of variably silicified and highly altered basalt enclosed in a matrix of pyrite-silica breccia or porous massive pyrite. A few pieces of chloritized basalt were recovered below the silicified basalt breccias. The silicified basalt and chloritized basalt breccias are underlain by slightly to moderately altered basalts, which exhibit chloritized alteration halos and contain veins of quartz, chlorite, and pyrite. Silicified basalt breccias recovered from the TAG-4 area resemble those in the upper part of the quartz-rich stockwork in the TAG-1 and TAG-2 areas, but they are generally less silicified and contain greater quantities of pyrite

Although abundant basaltic clasts were recovered, the framework-supported nature of the breccias, the absence of pervasive quartz and anhydrite cement, and the presence of only slightly to moderately underlying altered basaltic basement suggest that this area is not part of the high-temperature stockwork. Instead, these breccias appear to be part of a talus pile (Shipboard Scientific Party, 1996) adjacent to the main upflow zone that, measured from the basalt basement, is at least $20 \mathrm{~m}$ thick and is capped by a 10- to $15-\mathrm{m}-$ thick carapace of massive pyrite.

\section{TAG-5 Area (North Side of the Black Smoker Complex)}

The overall hydrothermal stratigraphy of the north side of TAG-5 is similar to that observed in the TAG-1 area to the east of the Black Smoker Complex. The upper part of the section is comprised dominantly of nodular pyrite- and pyrite-anhydrite breccias with minor chalcopyrite, similar to those in the anhydrite-rich zone in the TAG1 area. The hard, cherty material encountered in the upper few meters in the TAG-2, TAG-3, and TAG-4 areas was not recovered at TAG-5.

Below 30 mbsf, massive, coarse-grained granular pyrite is associated with the pyrite-silica breccias and contains significant amounts of silica. Remnant patches of chert, dark gray silica, and silicified altered basalt material are commonly included with the massive pyrite, suggesting that the rock formed by pyritization of a pre-existing pyrite-silica or a silicified basalt breccia. These breccias contain clasts of an earlier generation of pale-gray, fine-grained pyrite-silica breccia, indicating that there have been at least two stages of brecciation. Silicified basaltic breccias and basalt fragments in the lower $25 \mathrm{~m}$ are similar to stockwork samples from elsewhere beneath the mound, but they contain greater amounts of pyritic cement.

\section{MINERALOGICAL TYPES}

Samples selected for this study are representative of the homogeneous part of the sulfide-rich core. No specific sampling was made on the altered basalt. Mineralogical details of thin sections corresponding to the chemical analyses are presented in Table 1. Knott et al. Chap. 1, this volume), distinguish six facies or mineralogical types of samples (mineral assemblages with similar textural and mineralogical features: (1) Si-Fe oxyhydroxides, (2) porous $\mathrm{Fe}-(\mathrm{Zn}, \mathrm{Cu})$ sulfides, (3) massive granular Fe-sulfide, (4) sulfide-anhydrite, (5) sul- 
fide-quartz, (6) silicified/altered basalt. Most of these types are multistage breccias. We distinguish three major groups of samples. The first group forms the massive mound that is less than $20 \mathrm{~m}$ thick. Types 1,2, and 3 are in this group. These samples are massive, partly recrystallized sulfides with no silica and low anhydrite. They correspond closely to samples from the surface of the mound collected by submersible. The second group is primarily made of types 5 and 6 . The third group covers the anhydrite zone and can be considered as late anhydrite veining and impregnation in all the other types. These veins are common between 20 and $50 \mathrm{~m}$ depth and often associated with a chalcopyrite selvage. This sulfide precipitation within the mound contributes to the internal inflation of the mound.

\section{ANALYTICAL TECHNIQUES}

Samples were selected as homogeneous representative parts of the cores to focus our study on the chalcophile elements related to sulfides. For this study, we have not sampled massive anhydrite from the largest veins. The Leg 158 samples were studied for bulk analyses by X-ray fluorescence in IFREMER laboratories in Brest. First, samples were examined, cut into representative parts, and powdered. Analyses were made in two steps.

First, major elements, such as $\mathrm{Cu}, \mathrm{Fe}, \mathrm{Zn}, \mathrm{S}, \mathrm{Ca}, \mathrm{Ba}$, were analyzed on glass disks made of $0.2 \mathrm{~g}$ samples added to $11.3 \mathrm{~g}$ of a flux based on $\mathrm{Li}_{2} \mathrm{~B}_{4} \mathrm{O}_{7}-\mathrm{LiNO}_{3}-\mathrm{La}_{2} \mathrm{O}_{3}-\mathrm{NaBr}$. The glass disk was made by progressively heating the mixture in an electric furnace to $1000^{\circ} \mathrm{C}$, with a step of $40 \mathrm{~min}$ between $400^{\circ}$ and $500^{\circ} \mathrm{C}$ where oxidation of sulfides occurs. A problem in the fusion technique for sulfide analysis is the loss of $\mathrm{S}$ during the fusion, the use of this kind of flux allows $\mathrm{S}$ to be retained in the mixture. Detection limits for major elements is $0.1 \mathrm{wt} \%$ for $\mathrm{Pb}, \mathrm{Zn}$, and $\mathrm{Cu}, 0.2 \mathrm{wt} \%$ for $\mathrm{Ca}, 0.3 \mathrm{wt} \%$ for $\mathrm{SiO}_{2}, 0.5$ wt $\%$ for Fe, $\mathrm{S}$.

Minor and trace elements were analyzed using a different procedure. Because of the great dilution of the samples in the glass disk (1/ 86 ), which makes the detection of contents of less than $1 \mathrm{wt} \%$ difficult, trace element analyses were made on pellets. The pellets are composed of $6 \mathrm{~g}$ of sample powder thoroughly mixed with $0.6 \mathrm{~g}$ of wax, as a binding agent. This preparation is compressed under 10 tons pressure. This method has the advantages of simplicity, rapidity, and limited contamination. Because of the high concentration of major elements, matrix effects are important and they are resolved, in the case of this laboratory, by use of a Compton peak or by real calculation of matrix effects (for $\mathrm{Ni}, \mathrm{Co}$, and $\mathrm{Mn}$ ). Calibration was by the standard addition method checking certified standard samples. Detection limits for trace elements are $50 \mathrm{ppm}$ for $\mathrm{Mn}, 20 \mathrm{ppm}$ for $\mathrm{Pb}$ and $\mathrm{As}, 10 \mathrm{ppm}$ for $\mathrm{Sn}, \mathrm{Sr}, \mathrm{Ag}, \mathrm{Co}$, and Mo, 5 ppm for In and Ge, and $1 \mathrm{ppm}$ for Se.

\section{BULK CHEMISTRY, MAJOR ELEMENT VARIATION}

Analytical results are presented in Table 2. For each site all samples are listed according to increasing depth and results are illustrated in Figures 3-6. Some characteristics are common to all sites and will be described first. This will be followed by description of specific chemical characteristics for each site.

Base metal values are extremely low within the mound and stockwork zone below the near-surface material. High $\mathrm{Cu}$ values sporadically occur on the selvages of anhydrite veins within the anhydriterich zone. Base metals are enriched in the near-surface part of the mound.

Zinc concentrations are higher than $1 \mathrm{wt} \%$ in only $14 \%$ of the samples. Concentrations higher than $5 \mathrm{wt} \%$ occur in only $3 \%$ of the samples (Fig. 7). Except for two samples, most of the $\mathrm{Zn}$ is concentrated within the first $10 \mathrm{~m}$ in the upper part of the mound. All high values are in dark cherts, in porous massive pyrite, or in massive granular pyrite.

Copper concentrations are higher than $1 \mathrm{wt} \%$ in $52 \%$ of the samples, $15 \%$ of the samples have concentrations higher than $5 \mathrm{wt} \%$, and only $1 \%$ have concentrations higher than $20 \mathrm{wt} \%$. (Fig. 7). Copper is mostly concentrated in two types of samples: (1) in the porous pyrite breccia and porous massive pyrite at the surface (including one sample of massive granular chalcopyrite), and (2) related to anhydrite veins. More rarely, the pyrite-silica breccias are enriched in $\mathrm{Cu}$. There is no specific downward enrichment in $\mathrm{Cu}$. However, because of the different types of occurrence, high values are scattered through mound in different sample types.

Calcium occurs in anhydrite veins and associated impregnations in the different types of breccia from the active part of the mound. Calcium concentrations are higher than $1 \mathrm{wt} \%$ in $44 \%$ of the samples, $19 \%$ have concentration greater than $5 \mathrm{wt} \%$, and only one sample has a concentration greater than $20 \mathrm{wt} \%$. However, we did not sample the anhydrite veins. Highest values are between 20 and $40 \mathrm{mbsf}$ where the maximum number of veins were seen. Because of poor recovery, anhydrite (and thus Ca concentration) may be underestimated deeper in the mound. However, even if large veins were not sampled, the importance of anhydrite veining can be seen through the anhydrite impregnation in the nearby breccia and sulfides.

Silica concentration is higher than $1 \mathrm{wt} \%$ in $64 \%$ of the samples; $33 \%$ have concentrations greater than $10 \mathrm{wt} \%$ and $16 \%$ have concentrations greater than $30 \mathrm{wt} \%$. Thus, an important proportion of the mound is silica, occurring as opal or chert at the surface and as quartz within the mound. Except for the local occurrence of cherts near the surface, the first $20 \mathrm{~m}$ of the upper part of the mound is depleted in $\mathrm{SiO}_{2}$. There is no clear downward increasing trend for Si concentration.

Because $\mathrm{Al}$ and $\mathrm{Ti}$ are considered to be the immobile elements during hydrothermal alteration of oceanic basalt (Bideau et al., 1985), these two elements were measured to try to determine the importance of the basaltic component in the silicic breccia. In our collection, only one value is above the detection limit and thus does not allow the discussion of a basaltic origin of the silicic breccia.

\section{TAG-1}

The TAG-1 (Fig. 3) holes extend to $120 \mathrm{~m}$ deep and provide the most complete section through the mound. Calcium concentration has a relatively sharp increase at values close to $10 \mathrm{wt} \%$ at $20 \mathrm{~m}$ and then, if we do not consider the anhydrite vein samples, there is a regular decrease towards $50 \mathrm{~m}$, which is approximately the basement position (Fig. 3). This trend indicates a progressive decrease of anhydrite impregnation and, therefore, a decrease in the quantity of downgoing seawater. The decrease in $\mathrm{Ca}$ value may also reflect a progressive downward decrease of porosity, density, and size of veins. The Ca maximum may indicate the mixing front between seawater and ascending end-member hydrothermal fluid. The lack of $\mathrm{Ca}$ near the basement position at $45 \mathrm{~m}$ can be considered as a barrier for downgoing seawater, probably resulting from a lower permeability in the deepest part of the mound. This low permeability can be explained in two ways. First, mineral precipitates can lower the porosity of the breccia; second, the replacement of basaltic rocks by the pyrite/ silica assemblage gives a primary product with low permeability when compared to the mound. A possible explanation of the occurrence of anhydrite only at depths greater than $20 \mathrm{~m}$ is the position of the $160^{\circ} \mathrm{C}$ isotherm, which is the limit for anhydrite saturation in seawater (Blount and Dickson, 1969). Anhydrite blocks exposed on the seafloor are formed in the Black Smoker Complex where the $160^{\circ} \mathrm{C}$ isotherm reaches the seafloor (see Fig. 15).

Silica is not present in the surface massive pyrite. The amount of silica starts to increase progressively at $15 \mathrm{~m}$, with a sharp increase at 


\begin{tabular}{|c|c|c|c|c|c|c|c|c|c|c|c|c|c|c|c|}
\hline $\begin{array}{l}\text { Hole, core, section, } \\
\text { interval }(\mathrm{cm})\end{array}$ & $\begin{array}{l}\text { Depth } \\
\text { (mbsf) }\end{array}$ & Piece & Lithology & Pyrite & Marcasite & Sphalerite & Chalcopyrite & Anhydrite & Quartz & $\begin{array}{l}\text { Amorphous } \\
\text { silica }\end{array}$ & Hematite & Fe-oxides & Clay & Chlorite & Other \\
\hline \multicolumn{16}{|l|}{$\begin{array}{l}\text { TAG-1 } \\
158-957 \mathrm{C}-\end{array}$} \\
\hline $4 \mathrm{~W}-1,10-15$ & 10.58 & 3 & Nodular pyrite-anhydrite breccia & $\mathrm{D}$ & & $\operatorname{tr}$ & $\mathrm{m}$ & M & & & & & & & Dig \\
\hline $5 \mathrm{~N}-1,26-39$ & 15.25 & 5 & Massive pyrite (clast?) & $\mathrm{D}$ & & tr & $\mathrm{m}$ & $\mathrm{r}$ & $\operatorname{tr}$ & & & & $\operatorname{tr}$ & & \\
\hline $7 \mathrm{~N}-1,17-22$ & 19.66 & 5 & Nodular siliceous pyrite-anhydrite breccia & $\mathrm{M}$ & & tr & $\mathrm{m}$ & M & $\mathrm{A}$ & & & & $\operatorname{tr}$ & & \\
\hline $7 \mathrm{~N}-1,110-113$ & 20.60 & $8 \mathrm{D}$ & Nodular siliceous pyrite-anhydrite breccia & $\mathrm{D}$ & & tr & r & $\mathrm{M}$ & $\mathrm{m}$ & & & & U1 & & \\
\hline $7 \mathrm{~N}-2,58-63$ & 21.32 & $1 \mathrm{D}$ & Siliceous pyrite-anhydrite breccia & $\mathrm{M}$ & & tr & $\mathrm{r}$ & $\mathrm{M}$ & A & & & & & & \\
\hline $7 \mathrm{~N}-3,42-46$ & 22.61 & $4 \mathrm{~A}$ & $\begin{array}{l}\text { Siliceous pyrite-anhydrite breccia with } \\
\text { wallrock fragments }\end{array}$ & $\mathrm{M}$ & & tr & $\mathrm{r}$ & $\mathrm{M}$ & $\mathrm{m}$ & & & & $\operatorname{tr}$ & & \\
\hline $11 \mathrm{~N}-1,24-27$ & 30.88 & $3 \mathrm{~A}$ & Pyrite-silica breccia & $\mathrm{D}$ & & & $\operatorname{tr}$ & $\mathrm{m}$ & M & & & $\operatorname{tr}$ & & & \\
\hline $11 \mathrm{~N}-2,7-10$ & 32.19 & 1B & Pyrite-silica-anhydrite breccia & $\mathrm{M}$ & & $\operatorname{tr}$ & $\mathrm{r}$ & $\mathrm{m}$ & $\mathrm{M}$ & & & & $\operatorname{tr}$ & & \\
\hline $11 \mathrm{~N}-2,12-14$ & 32.19 & $1 \mathrm{C}$ & Massive pyrite-anhydrite breccia (vein related) & $\mathrm{D}$ & & tr & A & A & $\mathrm{r}$ & & & & $\operatorname{tr}$ & & \\
\hline $11 \mathrm{~N}-3,42-45$ & 33.97 & $5 \mathrm{~B}$ & Massive pyrite-chalcopyrite (vein related) & $\mathrm{D}$ & & tr & A & $\mathrm{M}$ & $\operatorname{tr}$ & & & & & & \\
\hline $11 \mathrm{~N}-3,54-57$ & 34.09 & $5 \mathrm{C}$ & Massive pyrite (vein related) & $\mathrm{D}$ & & tr & $\mathrm{r}$ & A & $\operatorname{tr}$ & & & & $\operatorname{tr}$ & & \\
\hline $11 \mathrm{~N}-3,123-126$ & 34.80 & $10 \mathrm{~B}$ & Pyrite-silica breccia & $\mathrm{M}$ & & tr & $\mathrm{r}$ & $\mathrm{m}$ & $\mathrm{D}$ & & & & $\operatorname{tr}$ & $\operatorname{tr}$ & \\
\hline $12 \mathrm{~N}-1,46-49$ & 35.35 & $5 \mathrm{C}$ & Massive pyrite-anhydrite breccia & $\mathrm{D}$ & & tr & A & A & $\mathrm{r}$ & & & & & $\operatorname{tr}$ & Po-morph? \\
\hline $13 \mathrm{~N}-1,12-20$ & 37.31 & 4 & Massive pyrite-anhydrite breccia & $\mathrm{D}$ & & tr & $\mathrm{r}$ & A & $\operatorname{tr}$ & & & & & tr & Galena? \\
\hline $13 \mathrm{~N}-1,13-20$ & 37.31 & 4 & Massive pyrite & $\mathrm{D}$ & & tr & $\mathrm{r}$ & $\mathrm{m}$ & $\mathrm{r}$ & & $\operatorname{tr}$ & & $\operatorname{tr}$ & tr & \\
\hline $13 \mathrm{~N}-1,109-112$ & 38.19 & $17 \mathrm{~B}$ & Pyrite-silica breccia & $\mathrm{D}$ & & tr & $\mathrm{r}$ & $\frac{\mathrm{m}}{\mathrm{m}}$ & $\mathrm{M}$ & & 1 & & $\operatorname{tr}$ & tr & \\
\hline $13 \mathrm{~N}-2,41-43$ & 38.89 & $3 \mathrm{D}$ & Pyrite-silica breccia & $\mathrm{M}$ & & tr & tr & ${ }_{\mathrm{r}}^{\mathrm{m}}$ & $\mathrm{D}$ & & & & tr & $\begin{array}{l}\text { II } \\
\text { tr }\end{array}$ & \\
\hline $14 \mathrm{~N}-1,18-22$ & 40.25 & 2 & Pyrite-silica breccia & $\mathrm{M}$ & & tr & r & $\mathrm{r}$ & $\mathrm{D}$ & & & $\operatorname{tr}$ & $\operatorname{tr}$ & tr & \\
\hline $14 \mathrm{~N}-2,44-47$ & 41.12 & $1 \mathrm{C}$ & Anhydrite vein & $\mathrm{A}$ & & tr & $\mathrm{r}$ & $\mathrm{D}$ & $\mathrm{tr}$ & & & II & II & $\pi$ & \\
\hline $15 \mathrm{~N}-1,110-114$ & 42.94 & 11D & Silicified wallrock breccia & $\mathrm{M}$ & & tr & tr & $\mathrm{m}$ & $\mathrm{D}$ & & & & $\operatorname{tr}$ & $\mathrm{r}$ & Rutile \\
\hline $15 \mathrm{~N}-2,3-6$ & 43.16 & $1 \mathrm{~A}$ & Pyrite-silica breccia & A & & tr & tr & $\mathrm{r}$ & $\mathrm{D}$ & & & & $\operatorname{tr}$ & tr & \\
\hline $15 \mathrm{~N}-4,47-50$ & 46.10 & 4 & Nodular pyrite-silica breccia & $\mathrm{M}$ & & & $\mathrm{r}$ & & $\mathrm{D}$ & & & & tr & & \\
\hline $16 \mathrm{~N}-1,119-121$ & 47.39 & 15 & Massive pyrite & $\mathrm{D}$ & & $\operatorname{tr}$ & $\mathrm{r}$ & $\mathrm{r}$ & $\mathrm{m}$ & & & & & & \\
\hline $16 \mathrm{~N}-2,52-55$ & 48.07 & $5 \mathrm{~A}$ & Silicified wallrock breccia & $\mathrm{D}$ & & tr & tr & $\mathrm{r}$ & A & & & & & tr & \\
\hline \multicolumn{16}{|l|}{ 158-957E- } \\
\hline $2 \mathrm{R}-1,23-28$ & 37.23 & 5 & Pyrite-silica breccia & $\mathrm{M}$ & & $\operatorname{tr}$ & $\mathrm{r}$ & $\mathrm{r}$ & $\mathrm{D}$ & & & & $\operatorname{tr}$ & & \\
\hline $5 \mathrm{R}-1,39-41$ & 58.99 & 8 & Pyrite-silica breccia & M & & tr & tr & $\operatorname{tr}$ & $\mathrm{D}$ & & & & $\operatorname{tr}$ & & Rutile \\
\hline $6 \mathrm{R}-1,4-6$ & 63.34 & 2 & Massive pyrite & $\mathrm{D}$ & & tr & A & & A & & & & $\mathrm{r}$ & & \\
\hline $8 \mathrm{R}-1,1-5$ & 72.80 & 1 & Massive pyrite & $\mathrm{D}$ & & tr & $\mathrm{r}$ & $\operatorname{tr}$ & A & & $\operatorname{tr}$ & & $\operatorname{tr}$ & & \\
\hline $11 \mathrm{R}-1,42-45$ & 87.52 & 9 & Pyrite-silica breccia & $\mathrm{D}$ & & tr & $\mathrm{r}$ & & $\mathrm{M}$ & & & & tr & & \\
\hline $14 \mathrm{R}-1,30-36$ & 101.80 & 8 & Pyrite-silica breccia & $\mathrm{M}$ & & $\operatorname{tr}$ & $\operatorname{tr}$ & & $\mathrm{D}$ & & $\operatorname{tr}$ & & $\mathrm{r}$ & & \\
\hline $17 \mathrm{R}-1,12-15$ & 116.15 & 2 & Pyrite-silica breccia & $\mathrm{D}$ & & $\operatorname{tr}$ & tr & $\mathrm{r}$ & $\mathrm{M}$ & & $\operatorname{tr}$ & & tr & & Leucox \\
\hline \multicolumn{16}{|l|}{ 158-957F- } \\
\hline $1 \mathrm{~N}-1,7-9$ & 1.05 & 2 & Massive granular chalcopyrite & & & $\operatorname{tr}$ & D & & $\operatorname{tr}$ & & & & & & Chalc. \\
\hline $1 \mathrm{~N}-1,41-46$ & 1.41 & $10 \mathrm{~A}$ & Pyrite-anhydrite breccia & $\mathrm{D}$ & & tr & A & $\mathrm{M}$ & $\operatorname{tr}$ & & & & $\operatorname{tr}$ & & \\
\hline $2 \mathrm{~N}-1,11-13$ & 5.60 & 3 & Massive granular pyrite & $\mathrm{D}$ & & tr & $\mathrm{r}$ & & $\operatorname{tr}$ & & & & $\operatorname{tr}$ & & \\
\hline \multicolumn{16}{|l|}{$158-957 \mathrm{G}-$} \\
\hline $1 \mathrm{~N}-1,36-38$ & 12.32 & 7 & Massive granular pyrite & $\mathrm{D}$ & & $\operatorname{tr}$ & $\mathrm{r}$ & & $\mathrm{r}$ & & & & $\operatorname{tr}$ & & \\
\hline $3 \mathrm{~N}-1,35-39$ & 21.35 & $4 \mathrm{~A}$ & Siliceous pyrite-anhydrite breccia & $\mathrm{M}$ & & $\operatorname{tr}$ & $\operatorname{tr}$ & $\mathrm{M}$ & $\mathrm{m}$ & & & $\operatorname{tr}$ & & & \\
\hline \multirow{2}{*}{\multicolumn{16}{|c|}{$\begin{array}{l}\text { TAG-2 } \\
158-957 \mathrm{~A}-\end{array}$}} \\
\hline & & & & & & & & & & & & & & & \\
\hline $3 \mathrm{X}-1,51-57$ & 10.50 & 11 & Porous massive pyrite & $\mathrm{D}$ & $\mathrm{r}$ & $\mathrm{m}$ & $\mathrm{r}$ & & & & & & & & $\mathrm{Cov}$ \\
\hline $1 \mathrm{R}-2,13-19$ & 1.13 & 2 & Massive granular pyrite & $\mathrm{D}$ & & & $\operatorname{tr}$ & & $\mathrm{r}$ & & & & $\operatorname{tr}$ & & \\
\hline \multicolumn{16}{|l|}{$158-957 \mathrm{H}-$} \\
\hline $1 \mathrm{~N}-1,60-70$ & 9.30 & 13 & Porous nodular pyrite breccia & $\mathrm{M}$ & & $\operatorname{tr}$ & A & $\mathrm{M}$ & & & & & & & \\
\hline $3 \mathrm{~N}-1,53-58$ & 18.23 & 12 & Pyrite-anhydrite breccia & $\mathrm{D}$ & & $\operatorname{tr}$ & $\mathrm{r}$ & A & $\operatorname{tr}$ & & & & & & \\
\hline $5 \mathrm{~N}-1,5-7$ & 26.70 & 1 & Massive granular pyrite & $\mathrm{D}$ & & & tr & & $\operatorname{tr}$ & & & & $\operatorname{tr}$ & & \\
\hline $5 \mathrm{~N}-2,80-84$ & 28.17 & $3 \mathrm{C}$ & $\begin{array}{l}\text { Pyrite-silica breccia } \\
\text { Prite }\end{array}$ & $\mathrm{M}$ & & $\operatorname{tr}$ & tr & $\mathrm{r}$ & $\mathrm{M}$ & & & & tr & & \\
\hline $7 \mathrm{~N}-1,7-13$ & 35.76 & 2 & Pyrite-silica breccia & $\mathrm{M}$ & & tr & $\mathrm{r}$ & $\mathrm{r}$ & $\mathrm{M}$ & & & & tr & & \\
\hline $8 \mathrm{~N}-1,97-107$ & 41.18 & 15 & Pyrite-silica breccia & $\mathrm{M}$ & & tr & $\mathrm{r}$ & $\mathrm{r}$ & $\mathrm{M}$ & & & $\mathrm{r}$ & tr & & \\
\hline $9 \mathrm{X}-1,15-18$ & $\begin{array}{l}41.18 \\
44.84\end{array}$ & 4 & Massive pyrite-silica & $\mathrm{D}$ & & tr & $\mathrm{r}$ & tr & A & & & & $\begin{array}{l}\text { ut } \\
\text { tr }\end{array}$ & & Chalc \\
\hline $1 \mathrm{~W}-1,11-14$ & 0.05 & 2 & Dark gray chert & $\mathrm{A}$ & $\operatorname{tr}$ & tr & tr & & $\mathrm{D}$ & & & $\operatorname{tr}$ & & & Bo; Dig; Cov; Chalc \\
\hline \multirow{2}{*}{\multicolumn{16}{|c|}{ TAG-4 }} \\
\hline 158-957I- & & & & & & & & & & $\operatorname{tr}$ & & & & & \\
\hline $\begin{array}{l}1 \mathrm{~N}-1,6-11 \\
1 \mathrm{~N}-1,64-67\end{array}$ & $\begin{array}{l}9.06 \\
9.60\end{array}$ & $\begin{array}{l}2 \\
10\end{array}$ & $\begin{array}{l}\text { Porous massive pyrite-marcasite } \\
\text { Massive pyrite }\end{array}$ & $\begin{array}{l}\mathrm{D} \\
\mathrm{D}\end{array}$ & $\begin{array}{l}\mathrm{m} \\
\mathrm{r}\end{array}$ & $\begin{array}{l}\mathrm{r} \\
\mathrm{r}\end{array}$ & $\begin{array}{l}\operatorname{tr} \\
\mathrm{r}\end{array}$ & & $\begin{array}{l}\operatorname{tr} \\
\operatorname{tr}\end{array}$ & $\operatorname{tr}$ & & & $\operatorname{tr}$ & & Bo; Cov \\
\hline $\begin{array}{l}158-957 \mathrm{~K}- \\
1 \mathrm{X}-1,35-38\end{array}$ & 030 & 5 & Massive pyrite & $\mathrm{D}$ & $\mathrm{m}$ & $r$ & $\mathrm{~m}$ & & $\operatorname{tr}$ & & & & & & Cov \\
\hline
\end{tabular}


Table 1 (continued).

\begin{tabular}{|c|c|c|c|c|c|c|c|c|c|c|c|c|c|c|c|}
\hline $\begin{array}{l}\text { Hole, core, section, } \\
\text { interval }(\mathrm{cm})\end{array}$ & $\begin{array}{l}\text { Depth } \\
\text { (mbsf) }\end{array}$ & Piece & Lithology & Pyrite & Marcasite & Sphalerite & Chalcopyrite & Anhydrite & Quartz & $\begin{array}{c}\text { Amorphous } \\
\text { silica }\end{array}$ & Hematite & Fe-oxides & Clay & Chlorite & Other \\
\hline $3 \mathrm{X}-1,2-7$ & 14.50 & 1 & Porous massive pyrite & $\mathrm{D}$ & $\mathrm{r}$ & $\mathrm{m}$ & $\operatorname{tr}$ & & A & & & & & & Chalc \\
\hline \multicolumn{16}{|l|}{$158-957 \mathrm{M}-$} \\
\hline $2 \mathrm{R}-1,15-20$ & 9.44 & 4 & Massive pyrite & M & $\mathrm{M}$ & r & $\mathrm{r}$ & & A & $\operatorname{tr}$ & & (1) & $\begin{array}{l}\text { Ir } \\
\operatorname{tr}\end{array}$ & & \\
\hline $3 \mathrm{R}-1,61-65$ & 14.90 & 15 & Pyrite-silica breccia & A & & tr & $\mathrm{r}$ & & $\mathrm{D}$ & & & & $\operatorname{tr}$ & & \\
\hline $3 \mathrm{R}-1,119-122$ & 15.49 & 28 & Pyrite-silica breccia & $\mathrm{D}$ & $\mathrm{r}$ & tr & $\mathrm{r}$ & & $\mathrm{M}$ & & & & $\operatorname{tr}$ & & \\
\hline $4 \mathrm{R}-1,41-46$ & 19.70 & 10 & Massive pyrite & $\mathrm{D}$ & $\mathrm{r}$ & $\mathrm{r}$ & A & & M & & & & tr & & \\
\hline $5 \mathrm{R}-1,93-97$ & 25.18 & 15 & Pyrite-silica breccia & $\mathrm{M}$ & $\mathrm{r}$ & tr & $\mathrm{r}$ & & $\mathrm{M}$ & & & & $\operatorname{tr}$ & & Cov \\
\hline $7 \mathrm{R}-1,8-12$ & 34.35 & 2 & Massive granular pyrite & $\mathrm{D}$ & & tr & $\mathrm{r}$ & & $\operatorname{tr}$ & & & & $\mathrm{m}$ & & \\
\hline $8 \mathrm{R}-1,8-12$ & 38.38 & 2 & Banded pyrite-marcasite & $\mathrm{D}$ & $\mathrm{m}$ & $\mathrm{r}$ & $\mathrm{r}$ & & A & & & & & & \\
\hline \multicolumn{16}{|l|}{$\begin{array}{l}\text { TAG-5 } \\
158-9570-\end{array}$} \\
\hline $2 \mathrm{R}-1,4-7$ & 7.93 & 2 & Pyrite-anhydrite breccia & $\mathrm{D}$ & & $\operatorname{tr}$ & $\mathrm{r}$ & $\mathrm{M}$ & & & & & & & Po-morph \\
\hline $2 \mathrm{R}-1,14-16$ & 8.03 & 4 & Massive pyrite-anhydrite & $\mathrm{D}$ & & tr & $\mathrm{r}$ & A & $\operatorname{tr}$ & & & & & & \\
\hline $4 \mathrm{R}-1,24-27$ & 16.13 & 6 & Pyrite-anhydrite breccia & $\mathrm{D}$ & & tr & $\mathrm{r}$ & $\mathrm{M}$ & $\operatorname{tr}$ & & & & & & \\
\hline \multicolumn{16}{|l|}{$158-957 \mathrm{P}-$} \\
\hline $1 \mathrm{R}-1,12-16$ & 0.11 & 3 & Pyrite-anhydrite breccia & $\mathrm{M}$ & & $\operatorname{tr}$ & $\mathrm{r}$ & $\mathrm{D}$ & & & & & & & Po-morph \\
\hline $3 \mathrm{R}-1,7-9$ & 11.95 & 2 & Massive granular pyrite & $\mathrm{D}$ & & tr & $\mathrm{r}$ & $\mathrm{m}$ & $\operatorname{tr}$ & & & & & & \\
\hline $5 \mathrm{R}-1,3-5$ & 21.50 & 1 & Massive pyrite-anhydrite & $\mathrm{D}$ & & tr & tr & $\mathrm{M}$ & $\mathrm{tr}$ & & & & & & Po-morph \\
\hline $6 \mathrm{R}-1,24-27$ & 26.72 & 5 & Massive granular pyrite & $\mathrm{D}$ & & tr & A & $\mathrm{tr}$ & $\mathrm{tr}$ & & & & $\operatorname{tr}$ & & Chalc \\
\hline $8 \mathrm{R}-1,23-26$ & 35.32 & 6 & Massive granular pyrite & $\mathrm{D}$ & & tr & $\mathrm{r}$ & & $\mathrm{r}$ & $\operatorname{tr}$ & & & $\mathrm{r}$ & $\operatorname{tr}$ & Chalc \\
\hline $8 \mathrm{R}-1,29-30$ & 35.37 & 7 & Porous massive pyrite & $\mathrm{D}$ & $\operatorname{tr}$ & $\mathrm{r}$ & A & & 1 & $\mathrm{r}$ & & & 1 & tu & Po \\
\hline $11 \mathrm{R}-1,3-6$ & 50.10 & 1 & Massive granular pyrite & $\mathrm{D}$ & & tr & $\mathrm{r}$ & $\mathrm{m}$ & & & & & & & \\
\hline $12 \mathrm{R}-2,54-57$ & 56.13 & 10 & Massive granular pyrite & $\mathrm{D}$ & & tr & tr & $\mathrm{m}$ & & & & & $\operatorname{tr}$ & & \\
\hline $12 \mathrm{R}-4,88-92$ & 57.96 & 11 & Massive pyrite-silica & $\mathrm{D}$ & & tr & tr & tr & A & & & & $\operatorname{tr}$ & & \\
\hline \multicolumn{16}{|l|}{ TAG-3 } \\
\hline $\begin{array}{c}158-957 \mathrm{Q}- \\
1 \mathrm{R}, 1-3\end{array}$ & 5.59 & cut. & Red chert & $\mathrm{r}$ & & & & & $\mathrm{D}$ & & & $\mathrm{M}$ & & & $\mathrm{Cov}$ \\
\hline
\end{tabular}

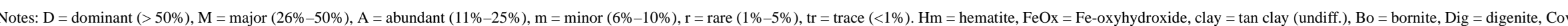
$=$ covellite, Po-morph $=$ pseudomorphs after pyrrhotite, Chalc $=$ chalcedonic or cryptocrystalline quartz texture, Leucox $=$ "leucoxene $"$ 


\begin{tabular}{|c|c|c|c|c|c|c|c|c|c|c|c|c|c|c|c|c|c|c|c|c|c|c|c|}
\hline $\begin{array}{l}\text { Hole, core, section, } \\
\text { interval }(\mathrm{cm})\end{array}$ & $\begin{array}{l}\text { Depth } \\
\text { (mbsf) }\end{array}$ & Piece & Type & Description & $\begin{array}{l}\mathrm{Cu} \\
(\%)\end{array}$ & $\begin{array}{l}\mathrm{Fe} \\
(\%)\end{array}$ & $\begin{array}{l}\mathrm{Zn} \\
(\%)\end{array}$ & $\begin{array}{c}\mathrm{S} \\
(\%)\end{array}$ & $\begin{array}{l}\mathrm{SiO}_{2} \\
(\%)\end{array}$ & $\begin{array}{l}\mathrm{Ca} \\
(\%)\end{array}$ & $\begin{array}{l}\mathrm{Al}_{2} \mathrm{O}_{3} \\
(\%)\end{array}$ & $\begin{array}{c}\mathrm{Pb} \\
(\mathrm{ppm})\end{array}$ & $\begin{array}{c}\mathrm{Sn} \\
(\mathrm{ppm})\end{array}$ & $\begin{array}{c}\mathrm{Mn} \\
(\mathrm{ppm})\end{array}$ & $\begin{array}{c}\mathrm{Sr} \\
(\mathrm{ppm})\end{array}$ & $\begin{array}{c}\mathrm{Ag} \\
(\mathrm{ppm})\end{array}$ & $\begin{array}{c}\text { As } \\
(\mathrm{ppm})\end{array}$ & $\begin{array}{l}\text { Co } \\
(\mathrm{ppm})\end{array}$ & $\begin{array}{l}\mathrm{Se} \\
(\mathrm{ppm})\end{array}$ & $\begin{array}{c}\mathrm{Cd} \\
(\mathrm{ppm})\end{array}$ & $\begin{array}{l}\text { Mo } \\
(\mathrm{ppm})\end{array}$ & $\begin{array}{c}\text { In } \\
(\mathrm{ppm})\end{array}$ & $\begin{array}{c}\mathrm{Ge} \\
(\mathrm{ppm})\end{array}$ \\
\hline \multicolumn{24}{|l|}{ TAG-1 } \\
\hline $\begin{array}{c}158-957 \mathrm{~F}- \\
1 \mathrm{~N}-1,7-9\end{array}$ & 1.07 & 2 & $5 \mathrm{~d}$ & $\begin{array}{l}\text { Massive granular } \\
\text { chalcopyrite }\end{array}$ & 31.85 & 30.3 & $<.1$ & 35.8 & 2.8 & $<.1$ & $<.4$ & 35 & $<10$ & $<50$ & $<10$ & 12 & $<20$ & 120 & 850.0 & $<10$ & 189 & $<5$ & 6 \\
\hline $1 \mathrm{~N}-1,41-46$ & 1.41 & $10 \mathrm{~A}$ & $6 a$ & $\begin{array}{l}\text { Nodular pyrite } \\
\text { breccia }\end{array}$ & 4.3 & 34.7 & $<.1$ & 46.1 & $<.3$ & 5.1 & $<.4$ & 32 & $<10$ & $<50$ & 520 & $<10$ & 30 & 380 & 18.3 & $<10$ & 83 & $<5$ & $<5$ \\
\hline $2 \mathrm{~N}-1,11-13$ & 5.61 & 3 & $5 \mathrm{c}$ & $\begin{array}{l}\text { Massive granular } \\
\text { pyrite }\end{array}$ & 0.44 & 45.0 & $<.1$ & 53.9 & 0.4 & $<.1$ & $<.4$ & 52 & $<10$ & $<50$ & $<10$ & $<10$ & 45 & 335 & 13.3 & $<10$ & 74 & $<5$ & $<5$ \\
\hline $\begin{array}{l}158-957 \mathrm{C}- \\
4 \mathrm{~W}-1,10-15\end{array}$ & 10.6 & 3 & $6 a$ & $\begin{array}{l}\text { Porous nodular } \\
\text { pyrite breccia }\end{array}$ & 5.7 & 31.6 & $<.1$ & 44.5 & $<.3$ & 7.1 & $<.4$ & 30 & $<10$ & $<50$ & 750 & $<10$ & 27 & 355 & 17.0 & $<10$ & 66 & $<5$ & $<5$ \\
\hline $\begin{array}{l}158-957 \mathrm{G}- \\
1 \mathrm{~N}-1,36-38\end{array}$ & 12.36 & 7 & $5 \mathrm{c}$ & $\begin{array}{l}\text { Massive granular } \\
\text { pyrite }\end{array}$ & 1.46 & 44.9 & 0.13 & 52.4 & 0.7 & $<.1$ & $<.4$ & 98 & $<10$ & $<50$ & $<10$ & $<10$ & 75 & 292 & 8.0 & $<10$ & 107 & $<5$ & $<5$ \\
\hline $\begin{array}{l}158-957 \mathrm{C}- \\
5 \mathrm{~N}-1,26-39\end{array}$ & 15.26 & 5 & $7 \mathrm{a}$ & $\begin{array}{l}\text { Massive pyrite- } \\
\text { anhydrite breccia }\end{array}$ & 1.06 & 44.3 & $<.1$ & 53.3 & 1.8 & 0.22 & $<.4$ & 50 & $<10$ & $<50$ & 20 & $<10$ & 55 & 430 & 17.9 & $<10$ & 76 & $<5$ & $<5$ \\
\hline $7 \mathrm{~N}-1,17-22$ & 19.67 & 5 & $7 \mathrm{c}$ & $\begin{array}{l}\text { Nodular siliceous } \\
\text { pyrite-anhydrite }\end{array}$ & 2.2 & 32.5 & $<.1$ & 45.6 & 4.3 & 6.65 & $<.4$ & 30 & $<10$ & $<50$ & 510 & $<10$ & 30 & 240 & 6.8 & $<10$ & 74 & $<5$ & $<5$ \\
\hline $7 \mathrm{~N}-1,110-113$ & 20.6 & $8 \mathrm{D}$ & $7 \mathrm{c}$ & $\begin{array}{l}\text { Nodular siliceous } \\
\text { pyrite-anhydrite } \\
\text { breccia }\end{array}$ & 0.87 & 26.2 & $<.1$ & 40.1 & 8.5 & 9.8 & $<.4$ & 20 & $<10$ & $<50$ & 620 & $<10$ & 30 & 130 & 3.4 & $<10$ & 42 & $<5$ & $<5$ \\
\hline $\begin{array}{l}158-957 \mathrm{G}- \\
3 \mathrm{~N}-1,35-39\end{array}$ & 21.35 & $4 \mathrm{~A}$ & $7 \mathrm{c}$ & $\begin{array}{l}\text { Nodular siliceous } \\
\text { pyrite-anhydrite } \\
\text { breccia }\end{array}$ & 0.58 & 36.6 & $<.1$ & 47.6 & 2.4 & 5.1 & $<.4$ & 45 & $<10$ & $<50$ & 430 & $<10$ & 40 & 470 & 12.7 & $<10$ & 55 & $<5$ & $<5$ \\
\hline $\begin{array}{l}158-957 \mathrm{C}- \\
7 \mathrm{~N}-2,58-63\end{array}$ & 21.43 & 1D & $7 \mathrm{c}$ & $\begin{array}{c}\text { Nodular siliceous } \\
\text { pyrite-anhydrite }\end{array}$ & 0.22 & 28.8 & $<.1$ & 44.0 & 1.6 & 10.5 & $<.4$ & 48 & $<10$ & $<50$ & 730 & $<10$ & 30 & 155 & 4.2 & $<10$ & 93 & $<5$ & $<5$ \\
\hline $7 \mathrm{~N}-3,42-46$ & 22.77 & $4 \mathrm{~A}$ & $7 \mathrm{~b}$ & $\begin{array}{l}\text { breccca } \\
\text { Nodular pyrite- } \\
\text { anhydrite breccia }\end{array}$ & 1.44 & 30.5 & $<.1$ & 44.2 & 3.4 & 8.4 & $<.4$ & 40 & $<10$ & $<50$ & 470 & $<10$ & 40 & 250 & 7.1 & $<10$ & 93 & $<5$ & $<5$ \\
\hline $11 \mathrm{~N}-1,24-27$ & 30.94 & $3 \mathrm{~A}$ & $9 a$ & $\begin{array}{l}\text { Pyrite-silica } \\
\text { breccia }\end{array}$ & 0.11 & 23.6 & $<.1$ & 30.8 & 39.8 & 2.98 & $<.4$ & $<20$ & $<10$ & $<50$ & 170 & $<10$ & $<20$ & 195 & 7.4 & $<10$ & 31 & $<5$ & $<5$ \\
\hline $11 \mathrm{~N}-2,7-10$ & 32.18 & $1 \mathrm{~B}$ & 8 & $\begin{array}{l}\text { Pyrite-silica- } \\
\text { anhydrite breccia }\end{array}$ & 2.38 & 28.1 & $<.1$ & 36.2 & 24.9 & 3.2 & $<.4$ & 25 & $<10$ & $<50$ & 195 & $<10$ & $<20$ & 245 & 12.8 & $<10$ & 45 & $<5$ & $<5$ \\
\hline $11 \mathrm{~N}-2,12-14$ & 32.23 & $1 \mathrm{C}$ & 8 & $\begin{array}{l}\text { Pyrite-silica- } \\
\text { anhydrite breccia }\end{array}$ & 7.02 & 34.7 & $<.1$ & 44.7 & 6.2 & 3.4 & $<.4$ & 40 & $<10$ & $<50$ & 225 & $<10$ & $<20$ & 200 & 11.4 & $<10$ & 81 & $<5$ & $<5$ \\
\hline $11 \mathrm{~N}-3,42-45$ & 34.03 & $5 \mathrm{~B}$ & 8 & $\begin{array}{l}\text { Pyrite-silica- } \\
\text { anhydrite breccia }\end{array}$ & 2.81 & 3.9 & $<.1$ & 33.7 & $<.3$ & 20.0 & $<.4$ & $<20$ & $<10$ & $<50$ & 1230 & $<10$ & $<20$ & 65 & 3.1 & $<10$ & 38 & $<5$ & $<5$ \\
\hline $11 \mathrm{~N}-3,54-57$ & 34.15 & $5 \mathrm{C}$ & 8 & $\begin{array}{l}\text { Pyrite-silica- } \\
\text { anhydrite breccia }\end{array}$ & 0.87 & 39.3 & 0.11 & 50.9 & $<.3$ & 3.83 & $<.4$ & 48 & $<10$ & $<50$ & 300 & $<10$ & 45 & 195 & 5.7 & $<10$ & 103 & $<5$ & $<5$ \\
\hline $11 \mathrm{~N}-3,123-126$ & 34.84 & $10 \mathrm{~B}$ & $9 a$ & $\begin{array}{l}\text { Pyrite-silica } \\
\text { breccia }\end{array}$ & 2.98 & 21.3 & $<.1$ & 30.5 & 30.7 & 6.0 & 0.8 & 30 & $<10$ & $<50$ & 400 & $<10$ & $<20$ & 240 & 12.4 & $<10$ & 49 & $<5$ & $<5$ \\
\hline $12 \mathrm{~N}-1,46-49$ & 35.66 & $5 \mathrm{C}$ & $7 \mathrm{a}$ & $\begin{array}{l}\text { Massive pyrite- } \\
\text { anhydrite breccia }\end{array}$ & 5.18 & 39.1 & $<.1$ & 49.3 & 1.3 & 2.7 & $<.4$ & 30 & $<10$ & $<50$ & 197 & $<10$ & 303 & 101 & 4.3 & $<10$ & 75 & $<5$ & $<5$ \\
\hline $\begin{array}{l}158-957 \mathrm{E}- \\
2 \mathrm{R}-1,23-28\end{array}$ & 37.23 & 5 & $9 b$ & $\begin{array}{l}\text { Nodular pyrite- } \\
\text { silica breccia }\end{array}$ & $<.1$ & 29.9 & $<.1$ & 36.4 & 32.6 & 0.9 & $<.4$ & 23 & $<10$ & $<50$ & 50 & $<10$ & $<20$ & 330 & 19.2 & $<10$ & 59 & $<5$ & $<5$ \\
\hline $\begin{array}{l}158-957 \mathrm{C}- \\
13 \mathrm{~N}-1,12-20\end{array}$ & 37.32 & 4 & $7 \mathrm{a}$ & $\begin{array}{l}\text { Massive pyrite- } \\
\text { anhydrite breccia }\end{array}$ & 0.36 & 35.1 & $<.1$ & 43.6 & 13.3 & 2.9 & $<.4$ & 30 & $<10$ & $<50$ & 165 & $<10$ & 30 & 345 & 13.8 & $<10$ & 97 & $<5$ & $<5$ \\
\hline $13 \mathrm{~N}-1,13-20$ & 37.33 & 4 & $7 \mathrm{a}$ & $\begin{array}{l}\text { Massive pyrite- } \\
\text { anhydrite breccia }\end{array}$ & 2.52 & 40.6 & $<.1$ & 50.9 & 1.7 & 2.5 & $<.4$ & 30 & $<10$ & $<50$ & 170 & $<10$ & 40 & 250 & 9.1 & $<10$ & 93 & $<5$ & $<5$ \\
\hline $13 \mathrm{~N}-1,109-112$ & 38.29 & $17 \mathrm{~B}$ & $9 a$ & $\begin{array}{l}\text { Pyrite-silica } \\
\text { breccia }\end{array}$ & 0.65 & 33.3 & $<.1$ & 43.4 & 13.3 & 4.0 & $<.4$ & 30 & $<10$ & $<50$ & 245 & $<10$ & 30 & 290 & 11.0 & $<10$ & 73 & $<5$ & $<5$ \\
\hline $13 \mathrm{~N}-2,41-43$ & 38.99 & $3 \mathrm{D}$ & $9 a$ & $\begin{array}{l}\text { Pyrite-silica } \\
\text { breccia }\end{array}$ & $<.1$ & 38.4 & $<.1$ & 47.8 & 8.2 & 2.18 & $<.4$ & 95 & $<10$ & $<50$ & 140 & $<10$ & 50 & 300 & 10.0 & $<10$ & 102 & $<5$ & $<5$ \\
\hline
\end{tabular}


Table 2 (continued).

\begin{tabular}{|c|c|c|c|c|c|c|c|c|c|c|c|c|c|c|c|c|c|c|c|c|c|c|c|}
\hline $\begin{array}{l}\text { Hole, core, section, } \\
\text { interval }(\mathrm{cm})\end{array}$ & $\begin{array}{l}\text { Depth } \\
\text { (mbsf) }\end{array}$ & Piece & Type & Description & $\begin{array}{l}\mathrm{Cu} \\
(\%)\end{array}$ & $\begin{array}{l}\mathrm{Fe} \\
(\%)\end{array}$ & $\begin{array}{l}\mathrm{Zn} \\
(\%)\end{array}$ & $\begin{array}{l}\mathrm{S} \\
(\%)\end{array}$ & $\begin{array}{l}\mathrm{SiO}_{2} \\
(\%)\end{array}$ & $\begin{array}{l}\mathrm{Ca} \\
(\%)\end{array}$ & $\begin{array}{l}\mathrm{Al}_{2} \mathrm{O}_{3} \\
(\%)\end{array}$ & $\begin{array}{c}\mathrm{Pb} \\
(\mathrm{ppm})\end{array}$ & $\begin{array}{c}\mathrm{Sn} \\
(\mathrm{ppm})\end{array}$ & $\begin{array}{l}\mathrm{Mn} \\
(\mathrm{ppm})\end{array}$ & $\begin{array}{c}\mathrm{Sr} \\
(\mathrm{ppm})\end{array}$ & $\begin{array}{c}\mathrm{Ag} \\
(\mathrm{ppm})\end{array}$ & $\begin{array}{l}\text { As } \\
(\mathrm{ppm})\end{array}$ & $\begin{array}{l}\text { Co } \\
(\mathrm{ppm})\end{array}$ & $\begin{array}{c}\mathrm{Se} \\
(\mathrm{ppm})\end{array}$ & $\begin{array}{c}\mathrm{Cd} \\
(\mathrm{ppm})\end{array}$ & $\begin{array}{l}\text { Mo } \\
(\mathrm{ppm})\end{array}$ & $\begin{array}{c}\mathrm{In} \\
(\mathrm{ppm})\end{array}$ & $\begin{array}{c}\mathrm{Ge} \\
(\mathrm{ppm})\end{array}$ \\
\hline $14 \mathrm{~N}-1,18-22$ & 40.38 & 2 & $9 \mathrm{a}$ & $\begin{array}{l}\text { Pyrite-silica } \\
\text { breccia }\end{array}$ & 2.47 & 42.8 & $<.1$ & 52.4 & 0.5 & 1.3 & $<.4$ & 40 & $<10$ & $<50$ & 90 & $<10$ & $<20$ & 245 & 15.6 & $<10$ & 101 & $<5$ & $<5$ \\
\hline $14 \mathrm{~N}-2,44-47$ & 41.39 & $1 \mathrm{C}$ & 11 & Anhydrite vein & 11.68 & 18.2 & $<.1$ & 34.9 & $<.3$ & 14.5 & $<.4$ & $<20$ & $<10$ & $<50$ & 920 & $<10$ & $<20$ & 80 & 10.6 & $<10$ & 24 & $<5$ & $<5$ \\
\hline $15 \mathrm{~N}-1,110-114$ & 43.3 & $11 \mathrm{D}$ & $10 \mathrm{a}$ & $\begin{array}{l}\text { Silicified wallrock } \\
\text { breccia }\end{array}$ & $<.1$ & 30.8 & $<.1$ & 38.9 & 23.6 & 2.5 & 0.4 & 30 & $<10$ & $<50$ & 150 & $<10$ & 30 & 210 & 8.0 & $<10$ & 79 & $<5$ & $<5$ \\
\hline $15 \mathrm{~N}-2,3-6$ & 43.43 & $1 \mathrm{~A}$ & 10a & $\begin{array}{l}\text { Silicified wallrock } \\
\text { breccia }\end{array}$ & $<.1$ & 41.3 & $<.1$ & 49.0 & 5.5 & 1.25 & $<.4$ & $<20$ & $<10$ & $<50$ & 75 & $<10$ & $<20$ & 1010 & 43.6 & $<10$ & 106 & $<5$ & $<5$ \\
\hline $15 \mathrm{~N}-4,47-50$ & 46.87 & 4 & $9 \mathrm{~b}$ & $\begin{array}{l}\text { Nodular pyrite- } \\
\text { silica breccia }\end{array}$ & $<.1$ & 27.0 & $<.1$ & 31.7 & 40.8 & $<.1$ & $<.4$ & $<20$ & $<10$ & $<50$ & $<10$ & $<10$ & $<20$ & 315 & 13.3 & $<10$ & 29 & $<5$ & $<5$ \\
\hline $16 \mathrm{~N}-1,119-121$ & 47.39 & 15 & 7 & $\begin{array}{l}\text { Pyrite-anhydrite } \\
\text { breccia }\end{array}$ & 6.00 & 36.6 & $<.1$ & 45.4 & 9.3 & 1.7 & $<.4$ & 35 & $<10$ & $<50$ & 95 & $<10$ & 22 & 335 & 22.9 & $<10$ & 60 & $<5$ & $<5$ \\
\hline $16 \mathrm{~N}-2,52-55$ & 48.14 & $5 \mathrm{~A}$ & $10 \mathrm{a}$ & $\begin{array}{l}\text { Silicified wallrock } \\
\text { breccia }\end{array}$ & 0.23 & 4.2 & $<.1$ & 41.3 & 22.8 & 0.6 & $<.4$ & 35 & $<10$ & $<50$ & 37 & $<10$ & 35 & 250 & 13.8 & $<10$ & 58 & $<5$ & $<5$ \\
\hline $\begin{array}{l}\text { 158-957E- } \\
5 \mathrm{R}-1,39-41\end{array}$ & 58.99 & 8 & $9 \mathrm{a}$ & $\begin{array}{l}\text { Pyrite-silica } \\
\text { breccia }\end{array}$ & 0.17 & 23.8 & $<.1$ & 28.2 & 48.8 & $<.1$ & $<.4$ & $<20$ & $<10$ & $<50$ & $<10$ & $<10$ & $<20$ & 330 & 16.5 & $<10$ & 32 & $<5$ & $<5$ \\
\hline $6 \mathrm{R}-1,4-6$ & 63.34 & 2 & $10 \mathrm{a}$ & $\begin{array}{l}\text { Silicified wallrock } \\
\text { breccia }\end{array}$ & 5.87 & 41.2 & $<.1$ & 49.3 & 4.7 & $<.1$ & $<.4$ & 57 & $<10$ & $<50$ & $<10$ & $<10$ & 35 & 140 & 14.6 & $<10$ & 75 & $<5$ & $<5$ \\
\hline $8 \mathrm{R}-1,1-5$ & 72.81 & 1 & $5 c$ & $\begin{array}{l}\text { Massive granular } \\
\text { pyrite }\end{array}$ & 2.74 & 42.6 & $<.1$ & 51.1 & 4.5 & $<.1$ & 0.4 & 30 & $<10$ & $<50$ & $<10$ & $<10$ & 40 & 220 & 21.1 & $<10$ & 80 & $<5$ & $<5$ \\
\hline $11 \mathrm{R}-1,42-45$ & 87.52 & 9 & $9 \mathrm{a}$ & $\begin{array}{l}\text { Pyrite-silica } \\
\text { breccia }\end{array}$ & 0.52 & 30.3 & $<.1$ & 35.9 & 34.1 & $<.1$ & $<.4$ & 75 & $<10$ & $<50$ & $<10$ & $<10$ & 25 & 265 & 14.3 & $<10$ & 85 & $<5$ & $<5$ \\
\hline $14 \mathrm{R}-1,30-36$ & 101.8 & 8 & $10 \mathrm{~b}$ & $\begin{array}{l}\text { Chloritized basalt } \\
\text { breccia }\end{array}$ & 0.16 & 34.8 & $<.1$ & 41.5 & 24.5 & $<.1$ & 0.4 & 35 & $<10$ & $<50$ & $<10$ & $<10$ & 35 & 1020 & 48.2 & $<10$ & 75 & $<5$ & $<5$ \\
\hline 17R-1, 12-15 & 116.22 & 2 & $10 \mathrm{~b}$ & $\begin{array}{l}\text { Chloritized basalt } \\
\text { breccia }\end{array}$ & $<.1$ & 36.5 & $<.1$ & 43.0 & 16.6 & 0.77 & $<.4$ & 65 & $<10$ & $<50$ & 65 & $<10$ & & 740 & & $<10$ & 93 & $<5$ & $<5$ \\
\hline $\begin{array}{l}\text { TAG-2 } \\
158-957 \mathrm{~N}- \\
1 \mathrm{~W}-1,11-14\end{array}$ & 0.11 & 2 & 3 & Dark gray chert & 0.78 & 11.0 & 1.81 & 13.8 & 73.0 & $<.1$ & $<.4$ & 90 & $<10$ & $<50$ & $<10$ & 17 & $<20$ & 20 & $<1$ & 45 & 17 & $<5$ & $<5$ \\
\hline $\begin{array}{l}\text { 158-957B- } \\
1 \mathrm{R}-1,48-53\end{array}$ & 0.48 & & & Drill cuttings & 11.00 & 29.4 & 2.48 & 33.3 & 17.2 & 0.78 & 0.45 & 47 & 46 & $<50$ & 65 & 193 & 68 & 65 & 6.4 & 92 & 54 & 17 & $<5$ \\
\hline $\begin{array}{l}\text { 158-957B- } \\
\text { 1R-2, 13-19 }\end{array}$ & 1.16 & 2 & $5 b$ & $\begin{array}{l}\text { Porous massive } \\
\text { sphalerite }\end{array}$ & $<.1$ & 44.3 & $<.1$ & 48.5 & 1.8 & $<.1$ & $<.4$ & 40 & $<10$ & $<50$ & $<10$ & $<10$ & $<20$ & 305 & 24.0 & $<10$ & 63 & $<5$ & 9 \\
\hline $\begin{array}{l}158-957 \mathrm{H}- \\
1 \mathrm{~N}-1,60-70\end{array}$ & 9.3 & 13 & $6 \mathrm{a}$ & $\begin{array}{r}\text { Porous nodular } \\
\text { pyrite breccia }\end{array}$ & 12.7 & 36.4 & $<.1$ & 45.7 & 0.5 & 2.5 & $<.4$ & 53 & $<10$ & $<50$ & 225 & $<10$ & 53 & 220 & 20.7 & $<10$ & 49 & $<5$ & $<5$ \\
\hline $\begin{array}{l}\text { 158-957A- } \\
3 \mathrm{X}-1,51-57\end{array}$ & 10.51 & 11 & $5 a$ & $\begin{array}{l}\text { Porous massive } \\
\text { pyrite }\end{array}$ & 0.6 & 33.9 & 154 & 8.0 & 2.5 & $<.2$ & $<.4$ & 360 & $<10$ & 65 & $<10$ & 56 & 85 & 8 & $<1$ & 200 & 93 & 5 & 48 \\
\hline $\begin{array}{l}158-957 \mathrm{H}- \\
3 \mathrm{~N}-1,53-58\end{array}$ & 18.23 & 12 & $6 a$ & $\begin{array}{l}\text { Porous nodular } \\
\text { pyrite breccia }\end{array}$ & 1.08 & 35.9 & $<.1$ & 48.1 & $<.4$ & 5.6 & $<.4$ & 25 & $<10$ & $<50$ & 450 & $<10$ & 38 & 285 & 12.7 & $<10$ & 50 & $<5$ & $<5$ \\
\hline $5 \mathrm{~N}-1,5-7$ & 26.75 & 1 & $5 c$ & $\begin{array}{l}\text { Massive granular } \\
\text { pyrite }\end{array}$ & $<.1$ & 44.8 & $<.1$ & 53.2 & 0.5 & $<.1$ & $<.4$ & $<20$ & $<10$ & $<50$ & $<10$ & $<10$ & 25 & 685 & 31.3 & $<10$ & 52 & $<5$ & $<5$ \\
\hline $5 \mathrm{~N}-2,80-84$ & 28.4 & $3 \mathrm{C}$ & $10 \mathrm{a}$ & $\begin{array}{l}\text { Silicified wallrock } \\
\text { breccia }\end{array}$ & $<.1$ & 37.5 & $<.1$ & 44.7 & 15.4 & 0.9 & $<.4$ & 37 & $<10$ & $<50$ & 95 & $<10$ & $<20$ & 315 & 16.5 & $<10$ & 66 & $<5$ & $<5$ \\
\hline $7 \mathrm{~N}-1,7-13$ & 35.77 & 2 & $9 \mathrm{~b}$ & $\begin{array}{l}\text { Nodular pyrite- } \\
\text { silica breccia }\end{array}$ & 0.12 & 31.4 & $<.1$ & 36.9 & 31.5 & $<.1$ & $<.4$ & 23 & $<10$ & $<50$ & $<10$ & $<10$ & 20 & 310 & 15.7 & $<10$ & 39 & $<5$ & $<5$ \\
\hline $8 \mathrm{~N}-1,97-107$ & 41.17 & 15 & $9 \mathrm{a}$ & $\begin{array}{l}\text { Pyrite-silica } \\
\text { breccia }\end{array}$ & 1.11 & 34.1 & $<.1$ & 39.2 & 24.3 & $<.1$ & $<.4$ & 40 & $<10$ & $<50$ & $<10$ & $<10$ & 27 & 535 & 24.4 & $<10$ & 63 & $<5$ & $<5$ \\
\hline $9 \mathrm{X}-1,15-18$ & 44.85 & 4 & $6 \mathrm{~b}$ & $\begin{array}{l}\text { Massive pyrite } \\
\text { breccia }\end{array}$ & 1.72 & 37.5 & $<.1$ & 45.0 & 14.4 & 0.9 & $<.4$ & 50 & $<10$ & $<50$ & 68 & $<10$ & 20 & 240 & 21.9 & $<10$ & 55 & $<5$ & $<5$ \\
\hline $\begin{array}{l}\text { TAG-3 } \\
\text { 158-957Q- } \\
\text { 1R-CC, } 1-13\end{array}$ & 5.68 & & - & Drill cuttings & 0.63 & 5.7 & 0.12 & 2.0 & 88.7 & 0.13 & $<.4$ & 28 & $<10$ & $<50$ & $<10$ & $<10$ & $<20$ & $<10$ & $<1$ & $<10$ & 6 & $<5$ & $<5$ \\
\hline
\end{tabular}


Table 2 (continued).

\begin{tabular}{|c|c|c|c|c|c|c|c|c|c|c|c|c|c|c|c|c|c|c|c|c|c|c|c|}
\hline $\begin{array}{l}\text { Hole, core, section, } \\
\text { interval }(\mathrm{cm})\end{array}$ & $\begin{array}{l}\text { Depth } \\
\text { (mbsf) }\end{array}$ & Piece & Type & Description & $\begin{array}{l}\mathrm{Cu} \\
(\%)\end{array}$ & $\begin{array}{l}\mathrm{Fe} \\
(\%)\end{array}$ & $\begin{array}{l}\mathrm{Zn} \\
(\%)\end{array}$ & $\begin{array}{c}\mathrm{S} \\
(\%)\end{array}$ & $\begin{array}{l}\mathrm{SiO}_{2} \\
(\%)\end{array}$ & $\begin{array}{c}\mathrm{Ca} \\
(\%)\end{array}$ & $\begin{array}{l}\mathrm{Al}_{2} \mathrm{O}_{3} \\
(\%)\end{array}$ & $\begin{array}{c}\mathrm{Pb} \\
(\mathrm{ppm})\end{array}$ & $\begin{array}{c}\mathrm{Sn} \\
(\mathrm{ppm})\end{array}$ & $\begin{array}{c}\mathrm{Mn} \\
(\mathrm{ppm})\end{array}$ & $\begin{array}{c}\mathrm{Sr} \\
(\mathrm{ppm})\end{array}$ & $\begin{array}{c}\mathrm{Ag} \\
(\mathrm{ppm})\end{array}$ & $\begin{array}{c}\text { As } \\
(\mathrm{ppm})\end{array}$ & $\begin{array}{c}\mathrm{Co} \\
(\mathrm{ppm})\end{array}$ & $\begin{array}{c}\mathrm{Se} \\
(\mathrm{ppm})\end{array}$ & $\begin{array}{c}\mathrm{Cd} \\
(\mathrm{ppm})\end{array}$ & $\begin{array}{c}\text { Mo } \\
(\mathrm{ppm})\end{array}$ & $\begin{array}{c}\text { In } \\
(\mathrm{ppm})\end{array}$ & $\begin{array}{c}\mathrm{Ge} \\
(\mathrm{ppm})\end{array}$ \\
\hline \multicolumn{24}{|l|}{$\begin{array}{l}\text { TAG-4 } \\
158-957 \mathrm{~K}-\end{array}$} \\
\hline $1 \mathrm{X}-1,35-38$ & 0.35 & 5 & $5 \mathrm{a}$ & $\begin{array}{l}\text { Porous massive } \\
\text { pyrite }\end{array}$ & 5.34 & 42.2 & 1.31 & 51.4 & $<.3$ & $<.1$ & $<.4$ & 200 & $<10$ & $<50$ & $<10$ & 32 & 95 & 130 & $<1$ & 32 & 218 & $<5$ & 6 \\
\hline $\begin{array}{c}158-957 \mathrm{M}- \\
1 \mathrm{R}-2,30-33\end{array}$ & 0.84 & 7 & $9 a$ & $\begin{array}{l}\text { Pyrite-silica } \\
\text { breccia }\end{array}$ & 1.98 & 24.1 & $<.1$ & 28.5 & 45.0 & $<.1$ & 0.6 & 25 & $<10$ & $<50$ & $<10$ & $<10$ & $<20$ & 270 & 10.8 & $<10$ & 26 & $<5$ & $<5$ \\
\hline $\begin{array}{l}158-957 \mathrm{I}- \\
1 \mathrm{~N}-1,6-11\end{array}$ & 9.06 & 2 & $5 \mathrm{a}$ & $\begin{array}{l}\text { Porous massive } \\
\text { pyrite }\end{array}$ & 0.12 & 42.9 & 3.84 & 52.9 & $<.3$ & $<.1$ & $<.4$ & 185 & $<10$ & $<50$ & $<10$ & 27 & 80 & 60 & 1.1 & 68 & 162 & $<5$ & 15 \\
\hline $\begin{array}{l}158-957 \mathrm{M}- \\
2 \mathrm{R}-1,15-20\end{array}$ & 9.45 & 4 & $5 a$ & $\begin{array}{l}\text { Porous massive } \\
\text { pyrite }\end{array}$ & $<.1$ & 43.5 & 1.98 & 52.4 & $<.3$ & $<.1$ & $<.4$ & 200 & $<10$ & $<50$ & $<10$ & 18 & 80 & 20 & 2.0 & 32 & 90 & $<5$ & 9 \\
\hline $\begin{array}{l}158-957 \mathrm{I}- \\
1 \mathrm{~N}-1,64-67\end{array}$ & 9.64 & 10 & $5 a$ & $\begin{array}{l}\text { Porous massive } \\
\text { pyrite }\end{array}$ & 3.41 & 43.3 & 1.05 & 52.4 & 0.7 & $<.1$ & $<.4$ & 230 & $<10$ & $<50$ & $<10$ & 63 & 78 & 280 & 8.5 & 23 & 97 & $<5$ & 10 \\
\hline $\begin{array}{l}158-957 \mathrm{~K}- \\
3 \mathrm{X}-1,2-7\end{array}$ & 14.52 & 1 & 5 & Massive pyrite & $<.1$ & 38.7 & 5.28 & 49.6 & 6.3 & $<.1$ & $<.4$ & 260 & $<10$ & $<50$ & $<10$ & 22 & 260 & 125 & 4.3 & 120 & 86 & $<5$ & 12 \\
\hline $\begin{array}{l}158-957 \mathrm{M}- \\
3 \mathrm{R}-1,61-65\end{array}$ & 14.91 & 15 & $9 a$ & $\begin{array}{l}\text { Pyrite-silica } \\
\text { breccia }\end{array}$ & 2.26 & 21.8 & $<.1$ & 25.9 & 50.4 & $<.1$ & $<.4$ & 20 & $<10$ & $<50$ & $<10$ & $<10$ & 22 & 275 & 8.0 & $<10$ & 42 & $<5$ & $<5$ \\
\hline $3 R-1,119-122$ & 15.49 & 28 & $9 a$ & $\begin{array}{l}\text { Pyrite-silica } \\
\text { breccia }\end{array}$ & 2.36 & 31.9 & 0.31 & 35.8 & 29.2 & $<.1$ & $<.4$ & 58 & $<10$ & $<50$ & $<10$ & $<10$ & 29 & 235 & 5.5 & 12 & 99 & $<5$ & $<5$ \\
\hline $4 \mathrm{R}-1,41-46$ & 19.71 & 10 & $5 a$ & $\begin{array}{l}\text { Porous massive } \\
\text { pyrite }\end{array}$ & 2.63 & 40.3 & 1.14 & 48.2 & 8.8 & $<.1$ & $<.4$ & 118 & $<10$ & $<50$ & $<10$ & 22 & 72 & 175 & 2.9 & 22 & 88 & $<5$ & 7 \\
\hline 5R-1, 93-97 & 25.23 & 15 & $9 a$ & $\begin{array}{l}\text { Pyrite-silica } \\
\text { breccia }\end{array}$ & 1.9 & 26.9 & $<.1$ & 32.1 & 40.5 & $<.1$ & 0.4 & 35 & $<10$ & $<50$ & $<10$ & $<10$ & 25 & 255 & 8.6 & $<10$ & 36 & $<5$ & $<5$ \\
\hline $7 \mathrm{R}-1,8-12$ & 34.38 & 2 & $5 \mathrm{c}$ & $\begin{array}{l}\text { Massive granular } \\
\text { pyrite }\end{array}$ & $<.1$ & 45.3 & 0.13 & 53.9 & 0.5 & $<.1$ & $<.4$ & 170 & $<10$ & $<50$ & $<10$ & $<10$ & 40 & 840 & 32.7 & 10 & 151 & $<5$ & $<5$ \\
\hline $8 \mathrm{R}-1,8-12$ & 38.38 & 2 & $5 \mathrm{c}$ & $\begin{array}{l}\text { Massive granular } \\
\text { pyrite }\end{array}$ & 0.58 & 41.2 & 5.45 & 1.1 & 2.0 & $<.1$ & $<.4$ & 137 & $<10$ & $<50$ & $<10$ & 23 & 80 & 95 & 3.3 & 125 & 95 & $<5$ & 15 \\
\hline \multicolumn{24}{|l|}{$\begin{array}{l}\text { TAG-5 } \\
158-957 \mathrm{P}\end{array}$} \\
\hline $1 \mathrm{R}-1,12-16$ & 0.12 & 3 & $7 \mathrm{a}$ & $\begin{array}{l}\text { Pyrite-anhydrite } \\
\text { breccia }\end{array}$ & 4.41 & 24.3 & $<.1$ & 40.2 & $<.4$ & 12.3 & $<.4$ & $<20$ & $<10$ & $<50$ & 860 & $<10$ & 23 & 140 & 13.6 & $<10$ & 54 & $<5$ & $<5$ \\
\hline $\begin{array}{r}158-957 \mathrm{O}- \\
2 \mathrm{R}-1,4-7\end{array}$ & 7.94 & 2 & $7 \mathrm{~b}$ & $\begin{array}{l}\text { Nodular pyrite- } \\
\text { anhydrite breccia }\end{array}$ & 3.11 & 33.9 & $<.1$ & 46.4 & $<.3$ & 6.82 & $<.4$ & 39 & $<10$ & $<50$ & 650 & $<10$ & 28 & 25 & 23.8 & $<10$ & 78 & $<5$ & $<5$ \\
\hline $2 \mathrm{R}-1,114-16$ & 8.04 & 4 & $7 \mathrm{~b}$ & $\begin{array}{l}\text { Nodular pyrite- } \\
\text { anhydrite breccia }\end{array}$ & 3.41 & 35.2 & $<.1$ & 46.6 & 0.6 & 5.7 & $<.4$ & 35 & $<10$ & $<50$ & 625 & $<10$ & 20 & 200 & 16.7 & $<10$ & 75 & $<5$ & $<5$ \\
\hline $\begin{array}{c}\text { 158-957P- } \\
3 \mathrm{R}-1,7-9\end{array}$ & 11.97 & 2 & $5 \mathrm{c}$ & $\begin{array}{l}\text { Massive granular } \\
\text { pyrite }\end{array}$ & 1.54 & 41.9 & 0.16 & 51.1 & $<.3$ & 1.95 & $<.4$ & 92 & $<10$ & $<50$ & 155 & $<10$ & 50 & 250 & 19.8 & $<10$ & 120 & $<5$ & $<5$ \\
\hline $13 \mathrm{~W}-1,37-42$ & 15.37 & & - & Drill cuttings & 8.3 & 40.4 & 0.19 & 48.8 & 2.2 & 0.31 & $<.4$ & 50 & $<10$ & $<50$ & 33 & 12 & 73 & 315 & 33.3 & 12 & 92 & $<5$ & $<5$ \\
\hline $\begin{array}{l}\text { 158-957O- } \\
4 \mathrm{R}-1,24-27\end{array}$ & 16.14 & 6 & $7 \mathrm{a}$ & $\begin{array}{l}\text { Nodular pyrite- } \\
\text { anhydrite breccia }\end{array}$ & 3.41 & 37.4 & $<.1$ & 48.4 & 0.6 & 4.4 & $<.4$ & 50 & $<10$ & $<50$ & 250 & $<10$ & 50 & 285 & 32.0 & $<10$ & 86 & 5 & $<5$ \\
\hline $\begin{array}{r}158-957 \mathrm{P}- \\
5 \mathrm{R}-1,3-5\end{array}$ & 21.53 & 1 & $5 c$ & $\begin{array}{l}\text { Massive granular } \\
\text { pyrite }\end{array}$ & $<.1$ & 38.4 & $<.1$ & 50.5 & 0.5 & 4.56 & $<.4$ & $<20$ & $<10$ & $<50$ & 350 & $<10$ & 30 & 1000 & 37.5 & $<10$ & 51 & $<5$ & $<5$ \\
\hline $6 \mathrm{R}-1,24-27$ & 26.74 & 5 & $5 \mathrm{c}$ & $\begin{array}{l}\text { Massive granular } \\
\text { pyrite }\end{array}$ & 3.83 & 44.3 & $<.1$ & 52.5 & $<.3$ & $<.1$ & $<.4$ & 30 & $<10$ & $<50$ & $<10$ & $<10$ & 60 & 175 & 32.6 & $<10$ & 120 & $<5$ & $<5$ \\
\hline $8 R-1,23-26$ & 35.33 & 6 & $5 \mathrm{c}$ & $\begin{array}{l}\text { Massive granular } \\
\text { pyrite }\end{array}$ & 0.41 & 44.8 & $<.1$ & 53.7 & 1.3 & $<.1$ & $<.4$ & 70 & $<10$ & 50 & $<10$ & $<10$ & 38 & 1270 & 60.4 & $<10$ & 103 & $<5$ & $<5$ \\
\hline
\end{tabular}


Table 2 (continued).

\begin{tabular}{|c|c|c|c|c|c|c|c|c|c|c|c|c|c|c|c|c|c|c|c|c|c|c|c|}
\hline $\begin{array}{l}\text { Hole, core, section, } \\
\text { interval }(\mathrm{cm})\end{array}$ & $\begin{array}{l}\text { Depth } \\
\text { (mbsf) }\end{array}$ & Piece & Type & Description & $\begin{array}{c}\mathrm{Cu} \\
(\%)\end{array}$ & $\begin{array}{l}\mathrm{Fe} \\
(\%)\end{array}$ & $\begin{array}{l}\mathrm{Zn} \\
(\%)\end{array}$ & $\begin{array}{c}\mathrm{S} \\
(\%)\end{array}$ & $\begin{array}{c}\mathrm{SiO}_{2} \\
(\%)\end{array}$ & $\begin{array}{c}\mathrm{Ca} \\
(\%)\end{array}$ & $\begin{array}{c}\mathrm{Al}_{2} \mathrm{O}_{3} \\
(\%)\end{array}$ & $\begin{array}{c}\mathrm{Pb} \\
(\mathrm{ppm})\end{array}$ & $\begin{array}{c}\mathrm{Sn} \\
(\mathrm{ppm})\end{array}$ & $\begin{array}{c}\mathrm{Mn} \\
(\mathrm{ppm})\end{array}$ & $\begin{array}{c}\mathrm{Sr} \\
(\mathrm{ppm})\end{array}$ & $\begin{array}{c}\mathrm{Ag} \\
(\mathrm{ppm})\end{array}$ & $\begin{array}{c}\text { As } \\
\text { (ppm) }\end{array}$ & $\begin{array}{c}\text { Co } \\
(\mathrm{ppm})\end{array}$ & $\begin{array}{c}\mathrm{Se} \\
(\mathrm{ppm})\end{array}$ & $\begin{array}{c}\mathrm{Cd} \\
(\mathrm{ppm})\end{array}$ & $\begin{array}{c}\text { Mo } \\
(\mathrm{ppm})\end{array}$ & $\begin{array}{c}\text { In } \\
(\mathrm{ppm})\end{array}$ & $\begin{array}{c}\mathrm{Ge} \\
(\mathrm{ppm})\end{array}$ \\
\hline 11R-1, 3-6 & 50.13 & 1 & $5 c$ & $\begin{array}{l}\text { Massive granular } \\
\text { pyrite }\end{array}$ & 2.81 & 41.9 & $<.1$ & 51.8 & 0.5 & 1.6 & $<.4$ & 30 & $<10$ & $<50$ & 250 & $<10$ & 47 & 1245 & 41.0 & $<10$ & 102 & $<5$ & $<5$ \\
\hline $12 \mathrm{R}-2,54-57$ & 56.18 & 10 & $5 c$ & $\begin{array}{l}\text { Massive granular } \\
\text { pyrite }\end{array}$ & 0.28 & 45.0 & $<.1$ & 53.7 & 1.0 & 0.28 & 0.6 & 40 & $<10$ & $<50$ & 40 & $<10$ & 59 & 885 & 42.2 & $<10$ & 123 & $<5$ & $<5$ \\
\hline $12 \mathrm{R}-4,88-92$ & 58.01 & 11 & $5 c$ & $\begin{array}{l}\text { Massive granular } \\
\text { pyrite }\end{array}$ & $<.1$ & 41.6 & $<.1$ & 48.8 & 7.9 & $<.1$ & $<.4$ & 80 & $<10$ & $<50$ & $<10$ & $<10$ & 63 & 355 & 14.2 & $<10$ & 101 & $<5$ & $<5$ \\
\hline
\end{tabular}

Notes: All samples: $\mathrm{Ba}<0.2 \%, \mathrm{~K}_{2} \mathrm{O}<0.06 \%, \mathrm{MgO}<0.4 \%, \mathrm{TiO}_{2}=<0.1 \% ; \mathrm{MnO}<0.03 \%, \mathrm{Ni}<20 \mathrm{ppm}, \mathrm{Sb}<15 \mathrm{ppm}$. All samples were analyzed by XRF at IFREMER. 
TAG-1

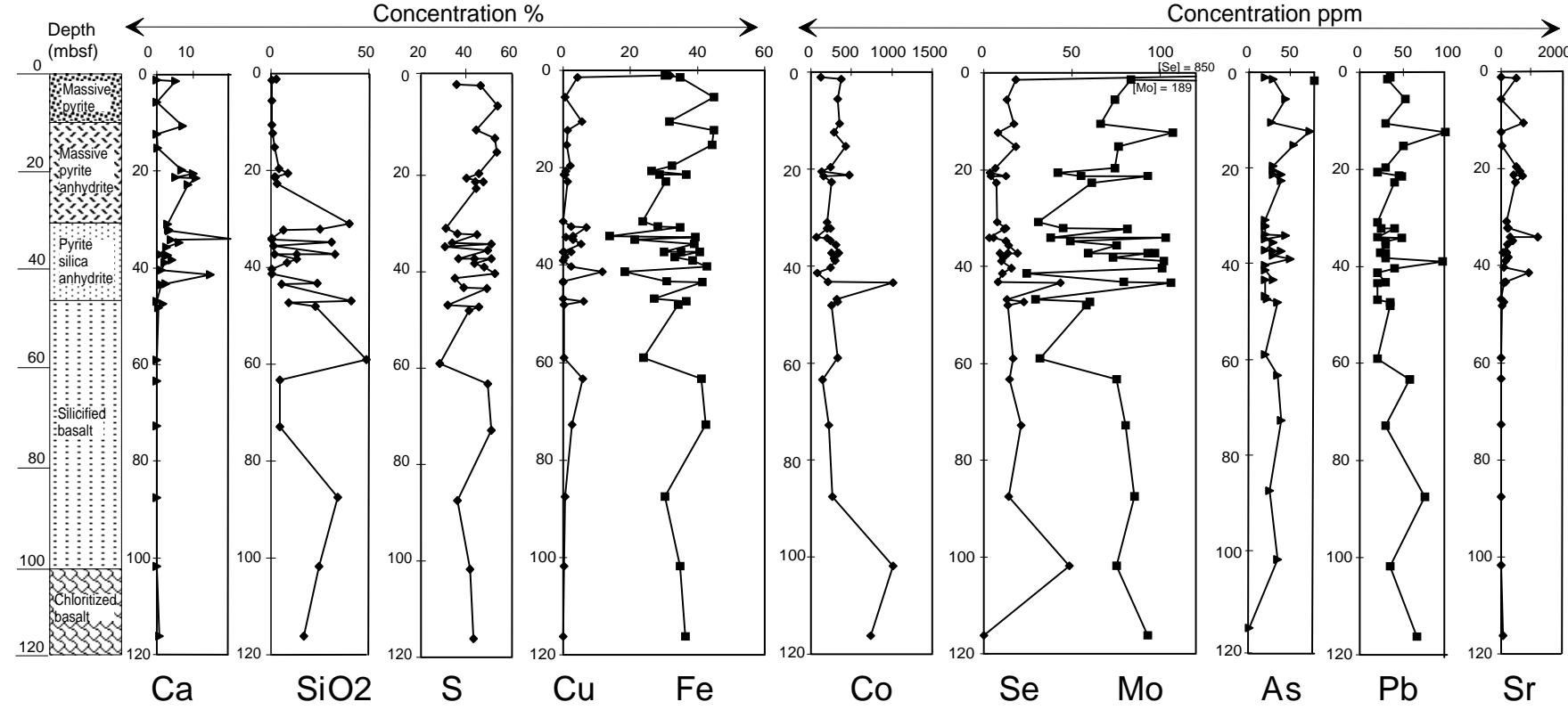

Figure 3. Chemical section of TAG-1.

TAG 2

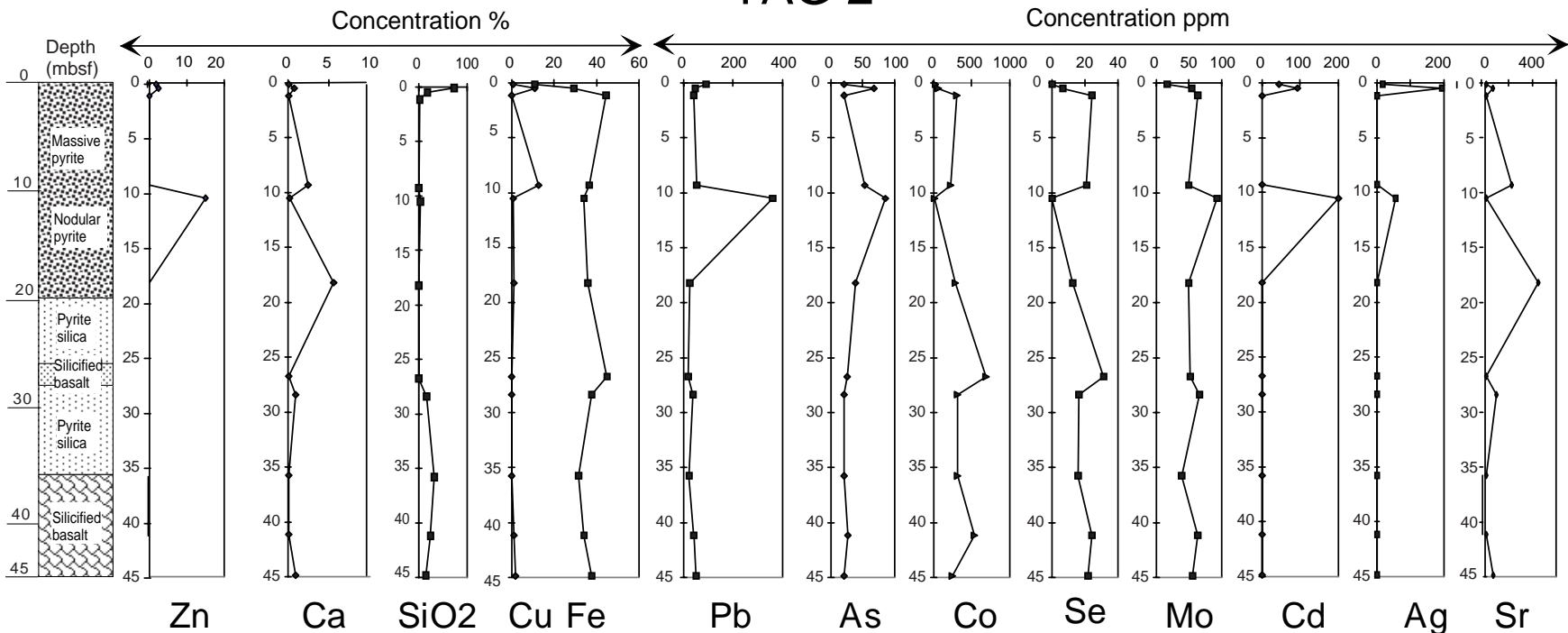

Figure 4. Chemical section of TAG-2.

about $30 \mathrm{~m}$ which is $10 \mathrm{~m}$ deeper than the Ca increase. Between 60 and $80 \mathrm{~m}$, Si concentrations are relatively low. At greater depth, silica is variable depending on the amount of basalt fragments present in the sample. As with $\mathrm{Ca}, \mathrm{Si}$ shows the first $20 \mathrm{~m}$ of the TAG deposits to be different from lower zones.

Zinc is highly depleted in the entire section of TAG-1: only two of the 37 samples have values just above $0.1 \mathrm{wt} \%$. In the upper $20 \mathrm{~m}$ that were probably formed as an accumulation of broken chimneys, we expected some $\mathrm{Zn}$ concentration in the samples. The absence of $\mathrm{Zn}$ in the central part of the mound and close to the black smoker complex may indicate the remobilization of $\mathrm{Zn}$.
The highest $\mathrm{Cu}$ concentrations are from a piece of massive chalcopyrite from the top of the deposit. This piece can be considered as a fragment of a black smoker. Except for this piece, $\mathrm{Cu}$ concentration is relatively low with local increases related to anhydrite veining in the anhydrite zone. The important point is the absence of high values and the absence of a downward $\mathrm{Cu}$ increase as generally seen in fossil deposits. In fact, the copper content is relatively low and homogeneous within the entire section. This may be explained by relatively stable physico-chemical conditions within the mound. The relation between $\mathrm{Cu}$ and $\mathrm{Ca}$ indicate that $\mathrm{Cu}$ is incorporated into the mound along the anhydrite veins and contributes to the internal inflation. 


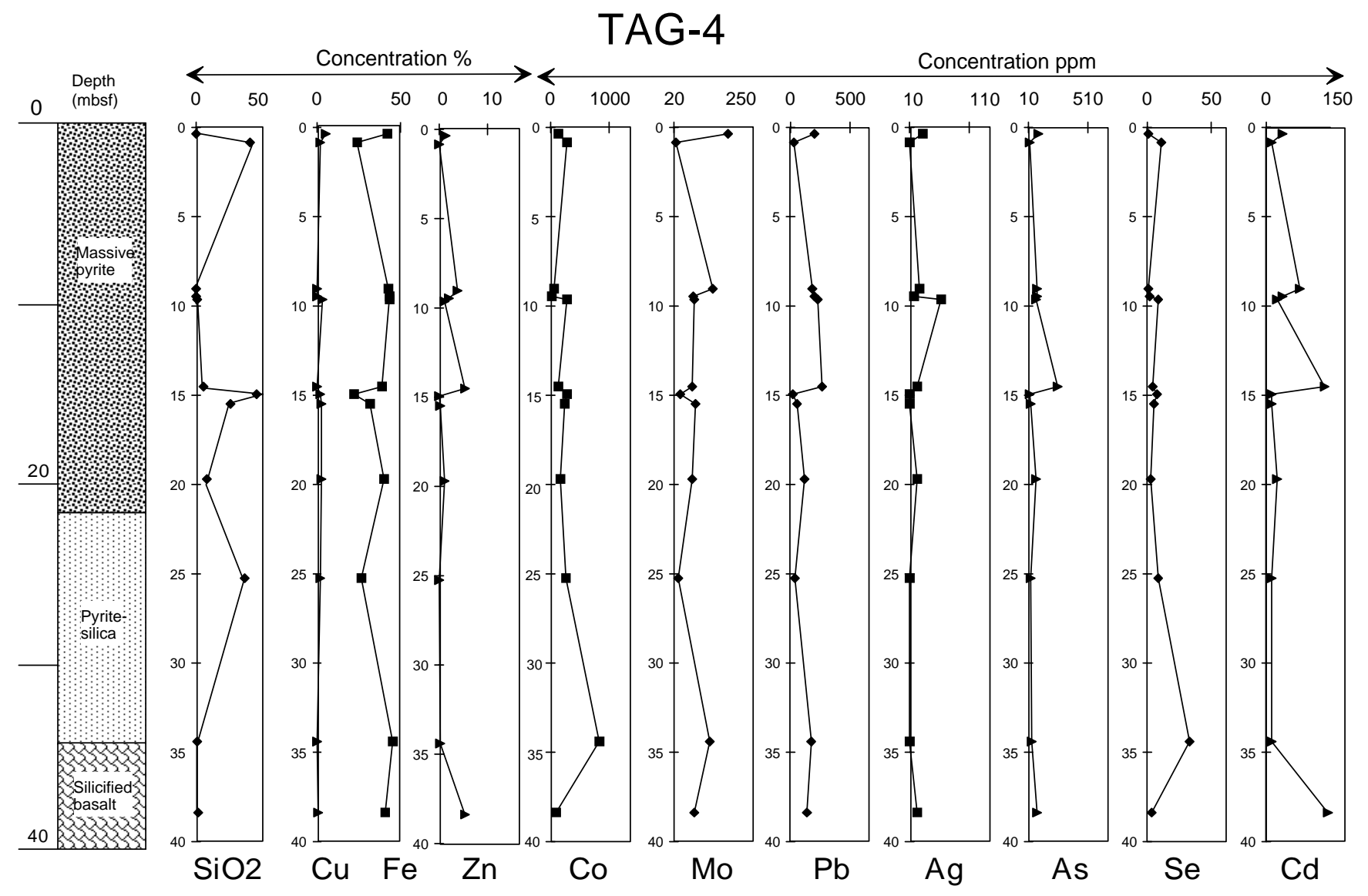

Figure 5. Chemical section of TAG-4.

The entire section in TAG-1 is extremely depleted in trace metals. In particular, trace metals such as $\mathrm{Cd}, \mathrm{Pb}, \mathrm{Ag}$, and $\mathrm{As}$ which are characteristic of low-temperature deposits are all at concentrations below or close to the detection limits (Table 2). Cobalt is relatively high, particularly in the deepest part of the section. Selenium is only concentrated in a black smoker chalcopyrite from the top of the section; this sample also has the highest Mo concentration.

\section{TAG-2}

TAG-2 (Fig. 4) is $40 \mathrm{~m}$ deep and allows the discussion of the lateral vertical zonation of the mound. Except for two samples, $\mathrm{Ca}$ is low, indicating either a less active part where anhydrite is not forming at present, or a cooler $\left(<160^{\circ} \mathrm{C}\right)$ but active part of the mound were anhydrite cannot precipitate. Silica is concentrated at two levels. In the upper part, silica is concentrated in grey cherts. This, together with the occurrence of a hard layer in this environment, may attest to the presence of a silica cap at the outer part of the mound. The second occurrence of silica is deeper in the mound with a sharp increase from 0 to $15 \mathrm{wt} \%$ at about $27 \mathrm{~m}$; the Si concentration then stabilizes at high concentrations. Zinc is concentrated in the first $10 \mathrm{~m}$, with values up to $15 \mathrm{wt} \%$. High values are found in the gray cherts, in the drill cuttings from the surfaces, or in porous massive pyrite. Below $10 \mathrm{~m}$, all values are below the detection limit. Thus, the important point here is the concentration of $\mathrm{Zn}$ in the first $10 \mathrm{~m}$ of the mound. Copper also has the two highest values (up to $12.70 \mathrm{wt} \%$ ) within the first $10 \mathrm{~m}$ of the mound. Other values are lower than $2 \mathrm{wt} \%$ and, as for TAG-1, there is no downward increase in copper concentration.
There is some concentration of $\mathrm{Cd}, \mathrm{Pb}, \mathrm{Ag}, \mathrm{Ge}$, and $\mathrm{As}$ in relation to local surficial $(<10 \mathrm{~m}) \mathrm{Zn}$ enrichment. The only indium value above the detection limit is from $\mathrm{Cu}-\mathrm{Zn}$ drill cuttings from the surface.

\section{TAG-4}

Silica is high in one sample taken from near the surface that contains basalt fragments and red chert fragments. At $15 \mathrm{~m}$ there is a sharp increase in $\mathrm{Si}$. The last $15 \mathrm{~m}$ of the section is massive pyrite depleted in silica. The presence of red cherts may attest, as for TAG-2, to the occurrence of a lateral silica cap at this site. A dual origin for silica can be proposed: chemical precipitation near surface and more deep-seated replacive silicification of basalt. Calcium is below the detection limit for all samples. The absence of anhydrite (Table 2) indicates that there is no mixing front in this part of the mound during the present episode of activity. This also accounts for a cooler part of the mound where the $160^{\circ} \mathrm{C}$ isotherm, necessary for the precipitation of anhydrite by heated seawater, is not reached. This is in good agreement with the negative heat flow and seawater inflow at this part of the mound (Becker and Von Herzen, 1996). Zinc is relatively concentrated in the massive pyrite and massive granular pyrite throughout the section and especially in the top $15 \mathrm{~m}$; the pyrite silica breccias, however, are depleted in $\mathrm{Zn}$. This, together with the preservation of primary mineralogical assemblages (such as collomorphic textures with pyrite and marcasite), indicates that the maturation is lower here than in other parts of the mound. Copper has relatively high values (up to $5.3 \mathrm{wt} \%$ ) both in the pyrite silica breccia and in the massive 


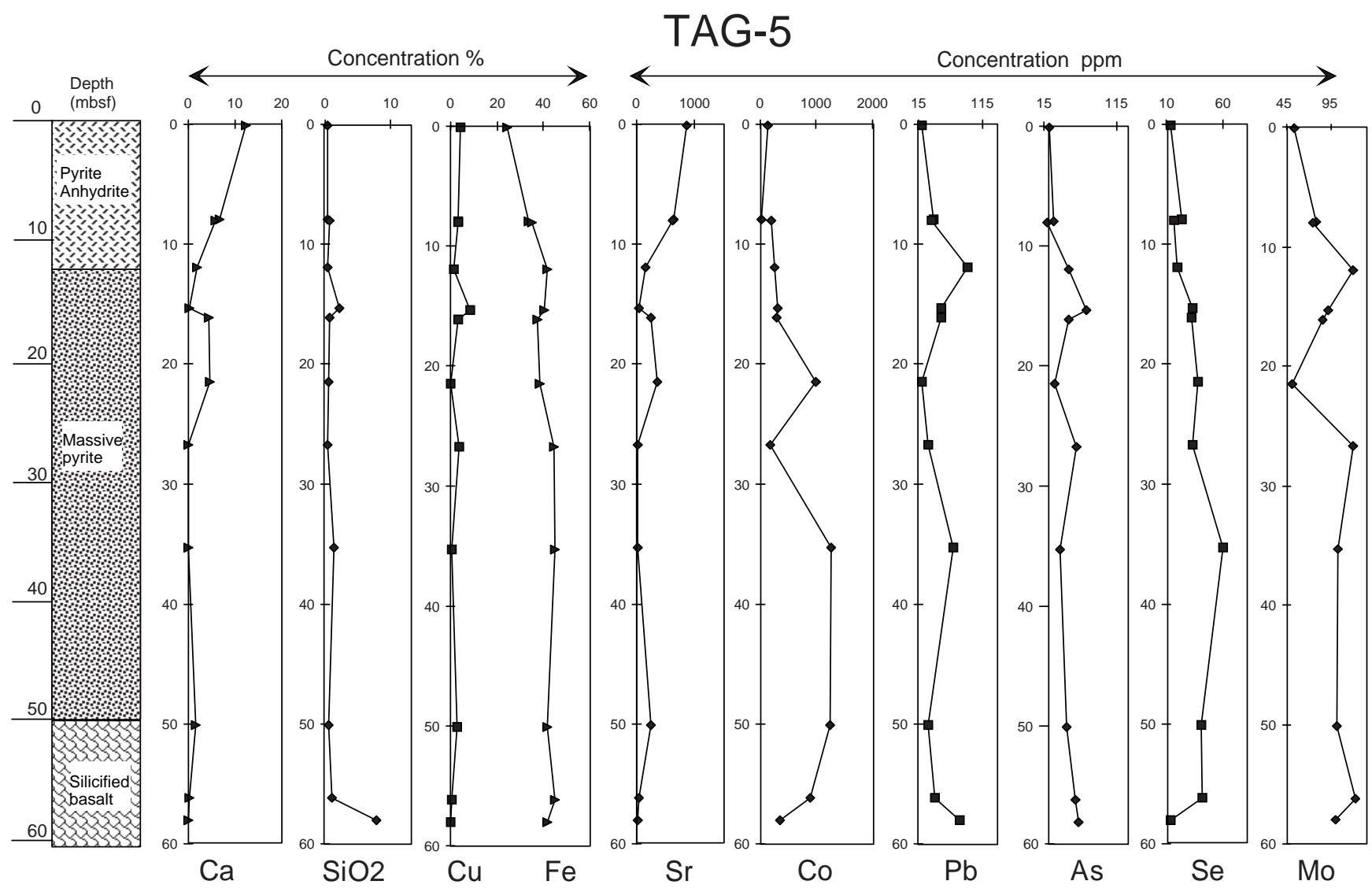

Figure 6. Chemical section of TAG-5.

granular pyrite in the whole section. As chalcopyrite is generally a late high-temperature mineral filling open spaces of the primary porous pyrite, not all the samples display this late $\mathrm{Cu}$-rich episode. Again, here there is no downward increase in the copper concentration.

Compared to other sites, TAG-4 (Fig. 5) is enriched in $\mathrm{Cd}, \mathrm{Pb}, \mathrm{Ag}$, $\mathrm{Ge}$, and As throughout the entire section. This is correlated to the $\mathrm{Zn}$ enrichment and the massive pyrite. High Co concentrations occur in the deep portion of the section. Compared to all other sections, $\mathrm{Se}$ is particularly low in TAG-4 (<10 ppm). Mo locally has the highest concentrations measured in our sample collection.

\section{TAG-5}

Calcium decreases progressively from $12.3 \mathrm{wt} \%$ at the surface to $0 \mathrm{wt} \%$ at $25 \mathrm{~m}$. This is different from TAG- 1 where Ca is not abundant at the surface. The occurrence of anhydrite at the surface indicates that, as with the black smoker, the mixing front here is close to the surface; in addition, the near surface temperature was close to $160^{\circ} \mathrm{C}$. One major point at TAG- 5 is that we did not observe the deep anhydrite veins. This is consistent with observation of well-preserved primary textures in sulfide that indicates relatively low temperature. This is also consistent with heat flow values indicating that the TAG5 area is close to the place where evidence of downgoing seawater in the mound was demonstrated (Becker et al., 1996). Silica is very low except in the last sample at $58 \mathrm{~m}$ depth. There is no sample with abundant silica; most samples are massive pyrite or nodular pyrite-anhydrite breccia. Copper is relatively high in all samples, and, as was the case for all other holes, there is no evidence for a downwards increase in copper. Zinc is extremely low, and only two values are close to the detection limit $(0.1 \mathrm{wt} \%)$. According to the mineralogical composi- tion, samples from this site could be of primary origin and do not correspond to recrystallization of porous marcasite rich sulfide as for TAG-4. In addition, the occurrence of pyrrhotite here is unique among the drilled samples and may indicate primary high-temperature assemblages.

As it was the case for TAG- $1, \mathrm{Cd}, \mathrm{Pb}, \mathrm{Ag}, \mathrm{As}$, and Ge concentrations are extremely low and close to the detection limit. Co is enriched in massive pyrite from the deepest part of the section; Se is also significantly enriched when compared to other sections, however these high values up to $60 \mathrm{ppm}$ are very low compared to the high values from the surface black smoker of TAG-1 (850 ppm).

\section{STATISTICAL ANALYSES ON BULK CHEMISTRY}

In order to better understand the behavior of elements and particularly trace elements we have calculated correlation (R) coefficient and made principal component analyses to see the affinity between the different elements and discuss the parameters that control the behavior of these elements. Calculations were made for each hole.

\section{Method: Correlation and Principal Component Analysis (PCA)}

The model of $\mathrm{R}$ calculation used in this study is the Pearson model (Beyer, 1968), which calculates the correlation for all the pairs of element columns. For two variables $x$ and $y$, the following formula is used:

$$
\frac{\Sigma(x-\bar{x})(y-\bar{y})}{(n-1) s_{1} s_{2}}
$$




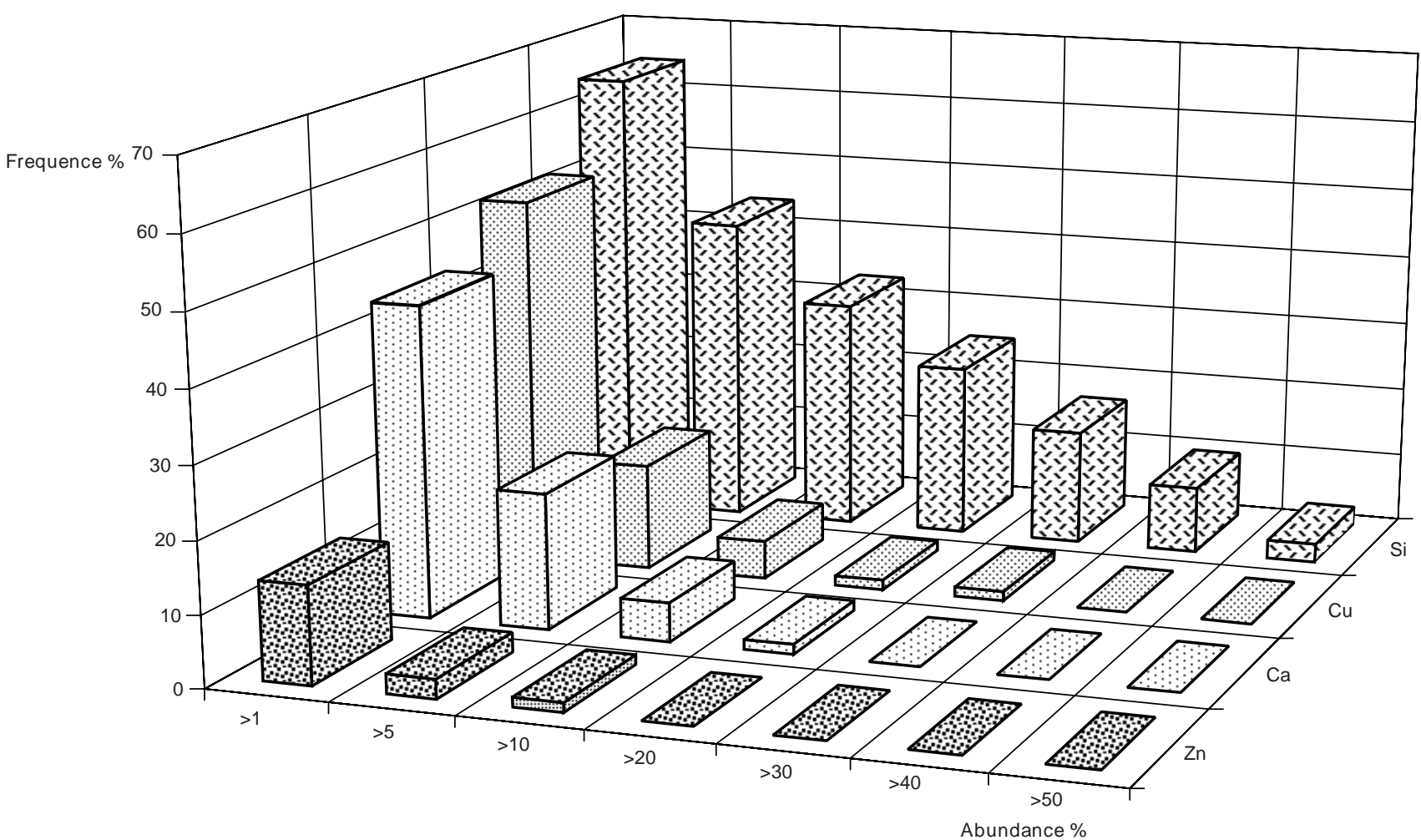

Figure 7. Relative enrichment of $\mathrm{Zn}, \mathrm{Ca}, \mathrm{Cu}$, and $\mathrm{Si}$ within the drilled part of the TAG mound. Note that $\mathrm{Zn}$ is at concentrations higher than 5 wt $\%$ in only $3 \%$ of the samples.

where $\bar{x}$ and $s_{1}, \bar{y}$ and $s_{2}$ are, respectively, the sample mean and standard deviation for the variables $x$ and $y$. In this case, the variables are the concentrations of each chemical elements for analyzed samples (e.g., $x=\mathrm{Co}, y=\mathrm{Fe}$ ).

PCA is a data reduction technique used to identify a small set of variables, known as the factors (or principal components), that account for a large proportion of the total variance in the original variables. In our case, the original variables are the chemical composition of the geological samples. Calculation is based on correlation matrix, the factors are used to highlight the differences in behavior of particular groups of elements under different conditions. The output consists of the proportion of the total variance explained by each factor, and the relative contribution of each chemical concentration to each principal component. These results are represented on graphs, which are the best way to illustrate similarities and differences expressed by the calculations. Each principal component is represented by an axis and, most often, the first 3 or 4 axes can explain more than $80 \%$ of the total variance. Elements close to the center of the two-dimensional plot (i.e., inertia center) cannot be discussed because they have a minimum contribution to both axes. Only elements close to the extremity of the axes are meaningful and used for the discussion. The difference in behavior of particular groups of elements can be explained by a good knowledge of the geological samples. For TAG-2, -3 , and -4 the population used for our statistical calculation is relatively low; thus, each sample has a higher importance in the calculation and one specific sample can have a strong weight in the calculation.

\section{Results}

The PCA diagram for TAG-1 (Fig. 8) shows four groups of elements. The first group (Co-Se) characterizes high-temperature precipitates (Fouquet et al., 1988). The second group comprises $\mathrm{Sr}$ and $\mathrm{Ca}$, related to anhydrite. The $\mathrm{Cu}$ position close to this group indicates a relation between high copper content and the anhydrite veining. This is consistent with macroscopic and microscopic observations indicating that chalcopyrite is enriched at the selvage of the anhydrite veins or disseminated as a late mineral near these veins. The lack of correlation between $\mathrm{Co}-\mathrm{Se}$ and $\mathrm{Cu}$ is not usual and may indicate that $\mathrm{Co}$ and Se are preferentially in pyrite rather than in the chalcopyrite related to the veins. The third group is Fe, Mo, S, As, and Sb. As and $\mathrm{Pb}$ are characteristic of low-temperature precipitates and are generally correlated with zinc in oceanic deposits (Fouquet et al., 1993b). The association of Mo with this group is unusual, but, in the absence of $\mathrm{Zn}$, these elements occur in pyrite. The high Mo-Fe coefficient ( $\mathrm{R}$ $\mathrm{Mo}-\mathrm{Fe}=0.76$ ). may just indicate a consistent association of pyrite with trace of molybdenite inclusions. The implication is probably a homogeneous source of fluids. The association of Fe and S with As and $\mathrm{Pb}$ also indicates that these elements are concentrated in pyrite. $\mathrm{SiO}_{2}$ is clearly independent of all other elements.

According to the knowledge we have of the samples, the following explanation can be proposed for the major contribution on the different axes. Axis 1 discriminates metallic sulfides on one side and sulfates and silica on the other side. The association of $\mathrm{Cu}$ and $\mathrm{Sr}-\mathrm{Ca}$ is a result of the specific relationship between chalcopyrite and anhydrite, and it indicates that this axis is also illustrative of anhydrite veining and thus mixing between hydrothermal fluid and seawater. Axis 2 can be regarded as the depth axis and illustrates the downward increase in $\mathrm{Co}, \mathrm{Se}$, and $\mathrm{Si}$. Axis 3 can be regarded as the temperature axis with an opposition between high-temperature $\mathrm{Co}, \mathrm{Se}, \mathrm{Cu}$, and $\mathrm{Sr}-\mathrm{Ca}$ and low-temperature $\mathrm{As}-\mathrm{Pb}$. The intermediate position of $\mathrm{Fe}-$ Mo-S along axis 3 is probably explained by the different temperature of formation of the pyrite. The $\mathrm{SiO}_{2}$ position is more difficult to explain, probably because two origins are possible for Si: chemical precipitation from the fluid and basalt replacement.

TAG-2 (Fig. 9) has the same association of elements as TAG-1. Because of the small number of samples, the results are more difficult to interpret. Se is associated with $\mathrm{Co}, \mathrm{Ca}$ is associated with $\mathrm{Sr}$, and $\mathrm{As}$ 


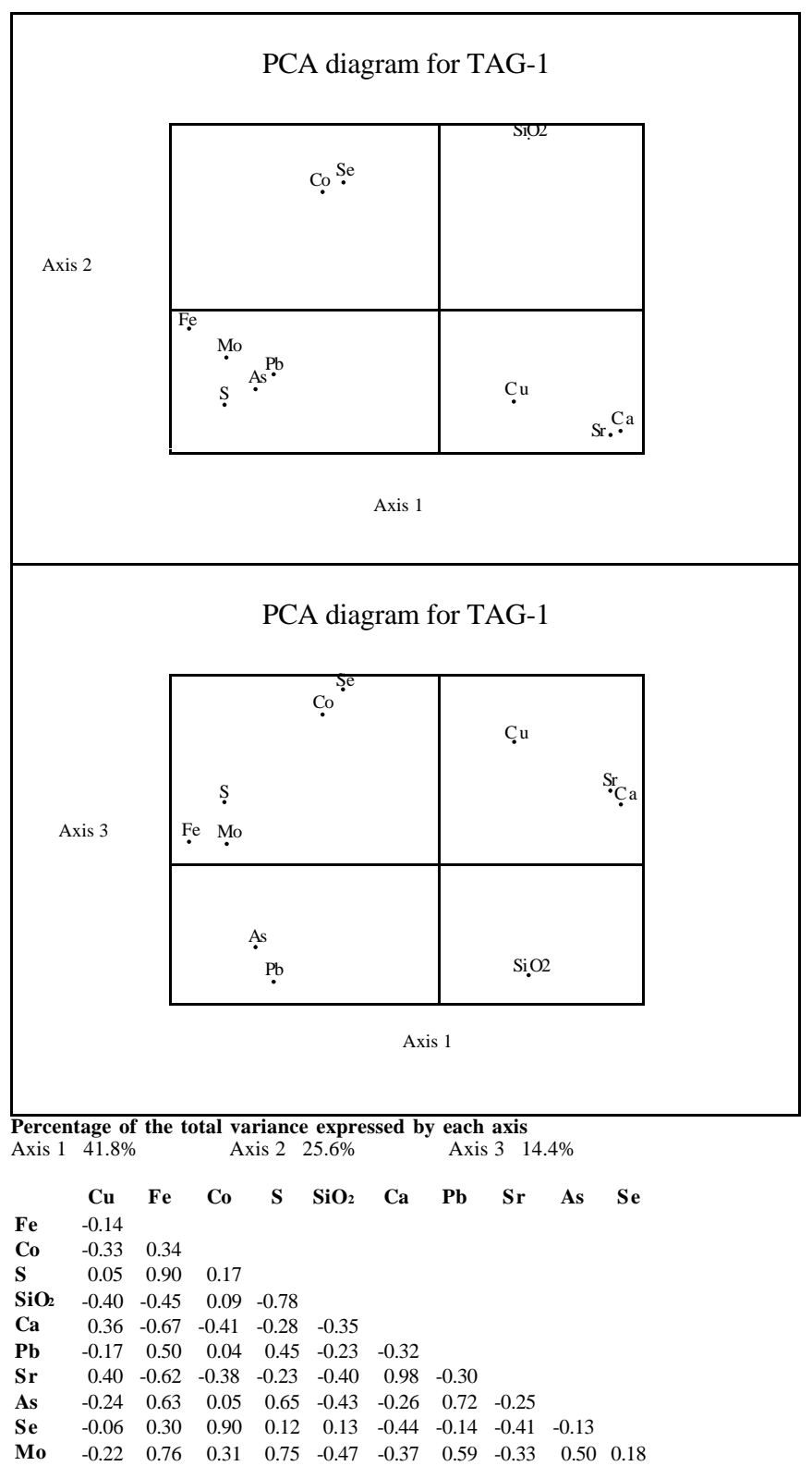

Figure 8. Principal component analyses diagrams and correlation matrix ( $\mathrm{R}$ values) for TAG-1. See text for explanation.

is associated with $\mathrm{Pb}$. Again, here $\mathrm{SiO}_{2}$ is independent of the other elements clusters. Along axis 1 is a strong opposition between $\mathrm{Fe}, \mathrm{Se}$, $\mathrm{Co}$, and $\mathrm{SiO}_{2}$. Axis 2 represents the depth contribution and illustrates the downwards $\mathrm{Se}, \mathrm{Co}$, and $\mathrm{Si}$ enrichment. This axis can also logically represent a downward increase in temperature. Several elements such as $\mathrm{Cu}, \mathrm{Ca}, \mathrm{Sr}$ close to the inertia center cannot be discussed precisely. A difference with TAG-1 is the position of Mo close to Ca-Sr. This is also seen in the lower correlation between Mo and $\mathrm{Fe}(\mathrm{R}=$ 0.586). $\mathrm{Cu}$ and $\mathrm{Ca}-\mathrm{Sr}$ along axis 3 illustrate veining/mixing.

In TAG-4, three groups are well defined along axes 1 and 2 (Fig. 10). $\mathrm{Zn}, \mathrm{Cd}$, and $\mathrm{As}$ are a typical association in $\mathrm{Zn}$-rich samples formed at medium to low temperature. $\mathrm{S}, \mathrm{Fe}, \mathrm{Mo}, \mathrm{Ag}$, and $\mathrm{Pb}$ are also related to this group. The major difference with TAG-1 and -2 is the preferential association of $\mathrm{As}$ with $\mathrm{Zn}$ rather than $\mathrm{Fe}$. This can be seen in the correlation coefficient, which is higher in TAG-1 (R As-Fe = 0.63 ) than in TAG-4 (R As-Fe $=0.4)$. The Pb-As correlation coefficient is the same, but these elements are at a higher concentration in

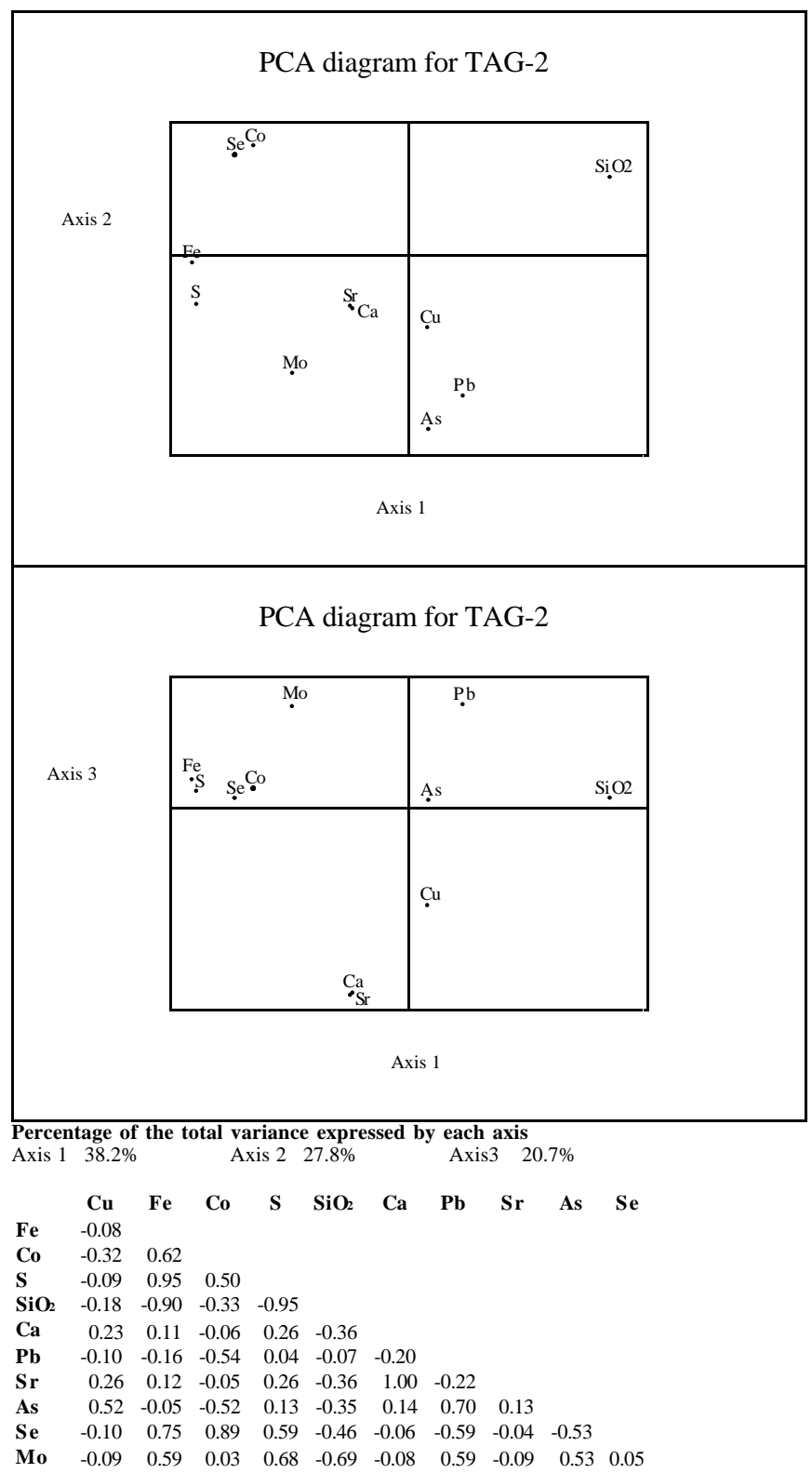

Figure 9. Principal component analyses diagrams and correlation matrix (R values) for TAG-2. See text for explanation.

TAG-4. This shows that, when $\mathrm{Zn}$ is present, As is preferentially associated with sphalerite rather than with pyrite. However, $\mathrm{Cu}$ is very close to the inertia center and thus cannot be discussed. $\mathrm{SiO}_{2}$ is independent of the other elements. As most of the silica occurs as primary quartz this may indicate that silica is also characteristic of high temperature; thus, axis 1 could be related to the temperature. According to the solubility of quartz in Fournier (1983), the hydrothermal solution is in equilibrium with quartz, which can thus precipitate easily when the temperature decreases. Axis 1 may also illustrate the Co, $\mathrm{Se}$, and $\mathrm{SiO}_{2}$ enrichment with depth. If part of the quartz corresponds to recrystallized low-temperature amorphous silica, axis 2 can also be related to temperature.

In TAG-5 (Fig. 11) on axes 1 and 2, As and $\mathrm{Pb}$ are again associated in the absence of sphalerite. Se and Co are correlated as for all other sites. $\mathrm{Cu}$ is intermediate between the sulfidic elements and the sulfate (Ca, Sr), as it was for all anhydrite-rich sites. This can be interpreted as $\mathrm{Cu}$ being a late element incorporated into the samples 


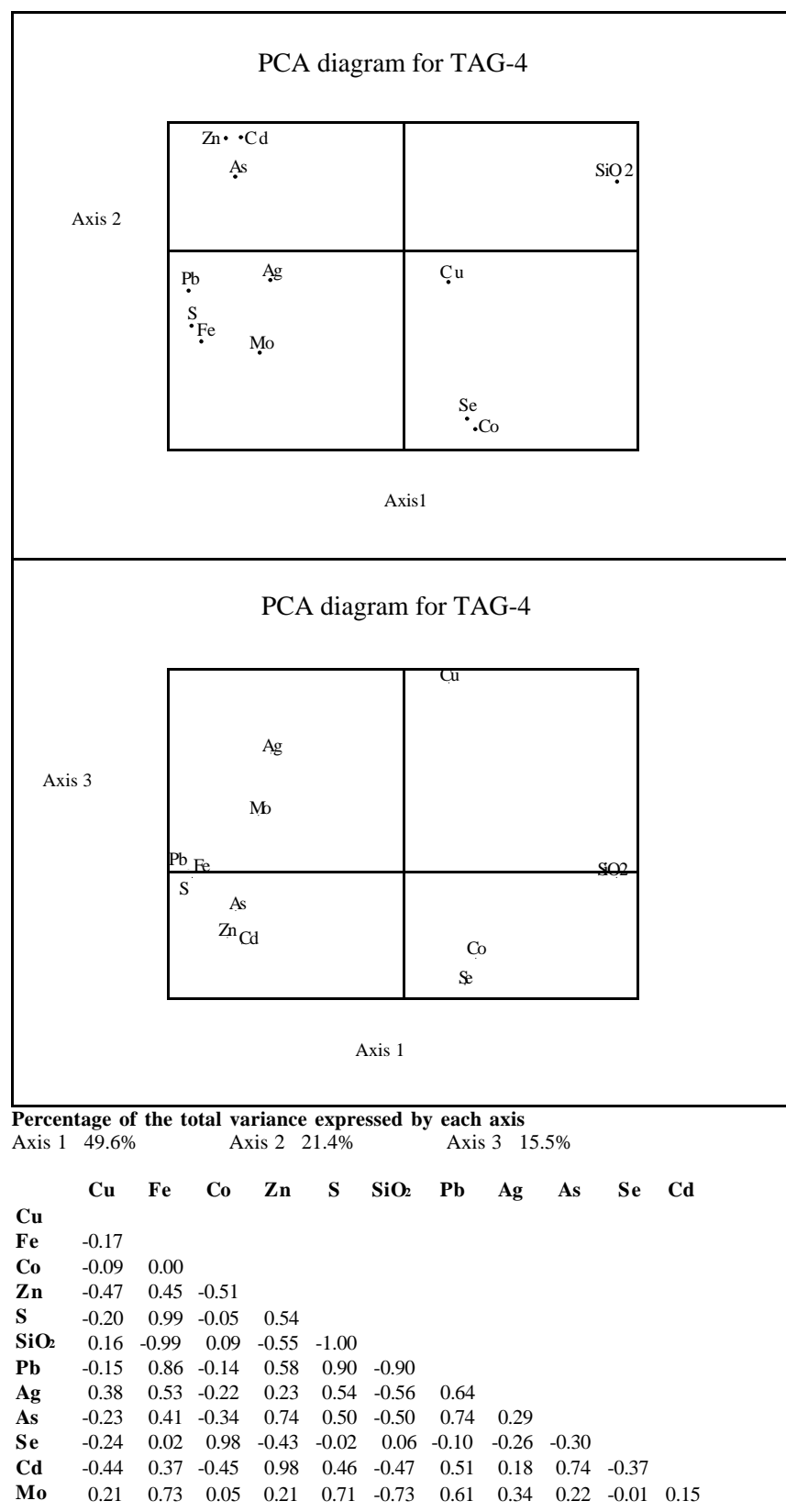

Figure 10. Principal component analyses diagrams and correlation matrix ( $R$ values) for TAG-4. See text for explanation.

during subsurface precipitation of chalcopyrite related to anhydrite. $\mathrm{SiO}_{2}$ is not very abundant and is closer to $\mathrm{As}$ and $\mathrm{Pb}$ than it was in all other sites. As usual, Mo is related to Fe and S. Axis 1 can be related to high-temperature sulfide/sulfate and veining/mixing within the mound. Axis 2 is probably the temperature axis. Axes 1 and 3 show for the first time a separation between $\mathrm{Co}$ and $\mathrm{Se} . \mathrm{Cu}$ is independent and $\mathrm{Ca}$ and $\mathrm{Sr}$ are as usual well correlated. $\mathrm{SiO}_{2}$ is associated with $\mathrm{Pb}$ and Co.

\section{COMPARISON WITH OTHER OCEANIC SITES}

In order to understand the chemical behavior of elements at TAG, we can compare our results with data from other oceanic sites in basaltic environments. The problem is that generally we only have surface samples from all other oceanic sites. At Snakepit, we have samples from the upper part of the stockwork, which indicate the classic

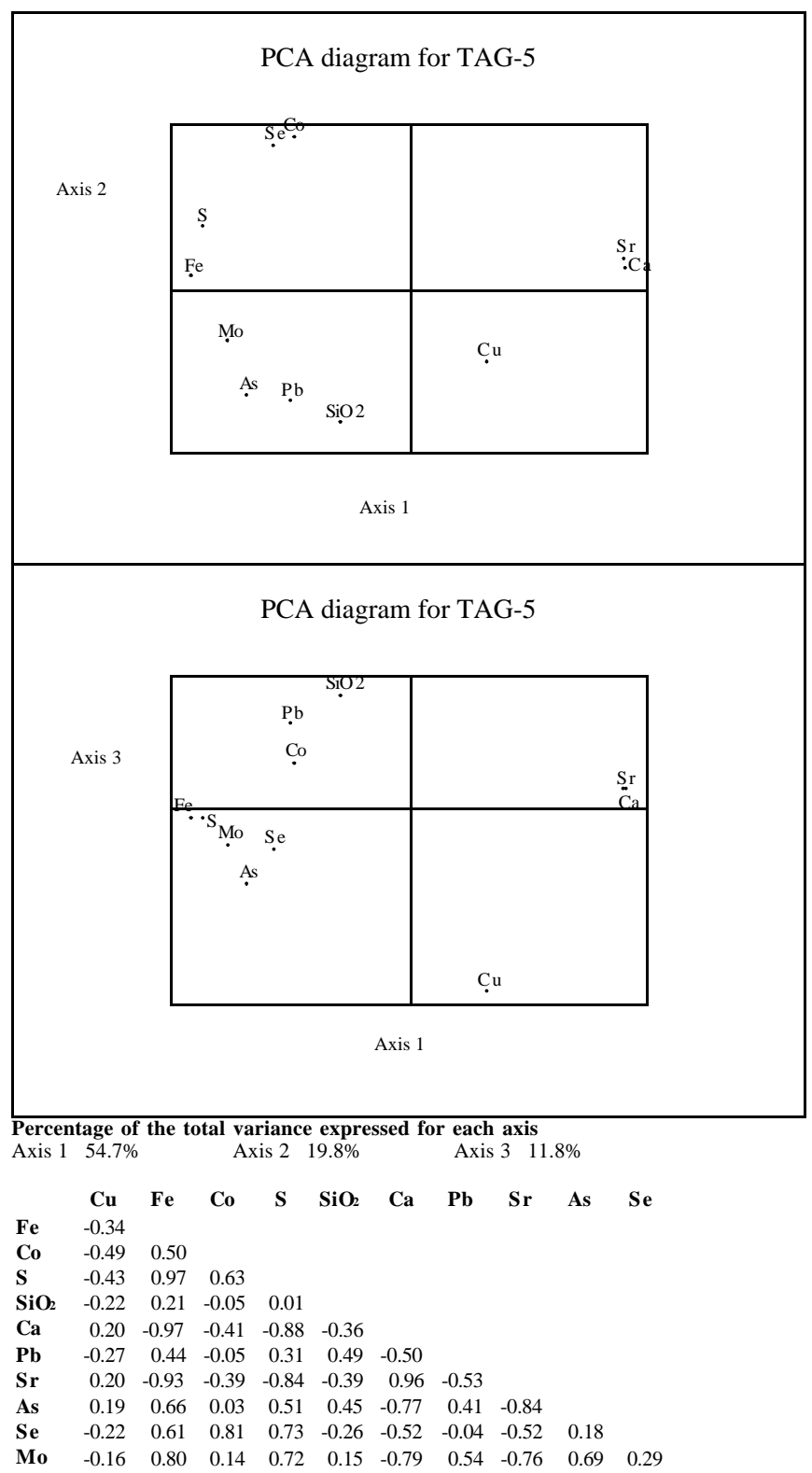

Figure 11. Principal component analyses diagrams and correlation matrix ( $\mathrm{R}$ values) for TAG-5. See text for explanation.

$\mathrm{Cu}$ enrichment and $\mathrm{Zn}$ depletion downwards. Another point is the depletion of $\mathrm{Zn}$ (and related trace elements) in the massive part of most mature mounds related to basalt. We present here a comparison of PCA diagrams for two major mounds similar in size to the TAG mound. One, the Snakepit mound, is on the Mid-Atlantic Ridge and the second one lies on a seamount near the East Pacific Rise at $13^{\circ} \mathrm{N}$. In addition we compare these results with data from young immature chimneys from the axial graben at $13^{\circ} \mathrm{N}$ on the East Pacific Rise. Samples on this sites were collected by submersibles on the mounds and along fault scarps in the mounds.

\section{Snakepit}

We have processed two groups of samples, one consisting of young chimneys and diffusers; the second of old mature massive sulfides from the surface and the core of the mound. Details of the geological setting, mineralogy, and chemistry of this deposit can be 
found in Fouquet et al. (1993b). The massive sulfide group is comparable with the upper part of the TAG mound.

\section{Young Chimneys and Diffusers}

In these young, immature samples, three groups of elements are distinguished along axis 1 , which represents the temperature contribution. The first group is typical of low- to medium- temperature diffusers. $\mathrm{Zn}, \mathrm{Sb}, \mathrm{Cd}, \mathrm{Au}$, and $\mathrm{Pb}$ are at the core of this group. $\mathrm{Ag}$ and As are close to this group (Fig. 12). The second group shows good correlation between $\mathrm{Cu}$ and $\mathrm{Se}(\mathrm{R}=0.95)$, $\mathrm{Co}$ is near this group but the Co-Se correlation is not high $(\mathrm{R}=0.52)$. The opposition between $\mathrm{S}$ and $\mathrm{Fe}$ is unusual, but can be explained by the rapid oxidation of pyrrhotite in the diffusers where pyrrhotite is the dominant Fe-mineral. In most inactive samples, pyrrhotite is oxidized into a mixture of lepidocrocite and native sulfur. As the stoichiometry of the mineral is destroyed, the correlation between $\mathrm{Fe}$ and $\mathrm{S}$ is no longer present. The intermediate position of $\mathrm{Si}$, occurring as late opal, can be explained by late pervasive precipitation of opal within pre-existing low- or high-temperature sulfide assemblages. We have often observed opal related to a late temperature fluid diffusing through the chimneys.

\section{Mature Massive Sulfide}

Again, here axis 1 represents the temperature contribution. The low-temperature group is $\mathrm{Cd}, \mathrm{Zn}, \mathrm{Ag}, \mathrm{Sb}, \mathrm{Au}$, and $\mathrm{Pb}$. As is independent of the group. The high-temperature group comprises $\mathrm{Cu}, \mathrm{Se}$, and $\mathrm{Co}$, the correlation between Se and Co is much higher $(\mathrm{R}=0.95)$ here than it was in the chimney group. In massive sulfide a good correlation is achieved between $\mathrm{Cu}$ and $\mathrm{Se}(\mathrm{R}=0.72)$ and $\mathrm{Cu}$ and $\mathrm{Co}(\mathrm{R}=$ 0.69). Along axis $1, \mathrm{Si}$ is closer to the low-temperature group than to the high-temperature one, which indicates preferential location of the late opal at the upper low-temperature part of the mound. Along axis $1, \mathrm{Fe}$ is in the high-temperature group, which may indicate dominant primary high-temperature pyrite. The intermediate position of As is different from that observed in the chimneys. A possible explanation is the occurrence of As as specific minerals such as jordanite and tennantite in chimneys and as substitution for $\mathrm{Fe}$ in pyrite from the massive sulfides.

\section{East Pacific Rise $13^{\circ} \mathbf{N}$}

At this site, a large deposit is located on a seamount close to the ridge axis. (Fouquet et al., 1996). Two groups of deposits are distinguished. The axial deposits correspond to very recent or active $\mathrm{Cu}-$ $\mathrm{Zn}$-rich chimneys. Off-axial deposits are from mature mounds and are primarily made of $\mathrm{Zn}$-poor massive sulfides. Geological setting and chemical analyses of sulfides from this field are presented in Fouquet et al. (1988, 1996). For this work we have separately processed the axial and off-axial group of samples to discuss the behavior of trace elements in young, immature deposits with respect to the older mature mounds.

\section{Axial Immature Chimneys From the Axial Graben}

Axis 1 represents the temperature contribution (Fig. 13). The association of $\mathrm{Ca}$ and $\mathrm{Sr}$ with $\mathrm{Cu}$ and $\mathrm{Se}$ is compatible with the observation of both chalcopyrite and anhydrite in the high-temperature black smoker chimneys. Lead and Ag are close to $\mathrm{Zn}-\mathrm{Cd}$ in the lowtemperature group. The occurrence of $\mathrm{Pb}$ close to $\mathrm{SiO}_{2}(\mathrm{R}=0.99)$ is remarkable and well explained by the systematic occurrence of galena at the edge of dendritic sphalerite embedded in silica at the outermost and lower temperature part of the chimneys. The occurrence of jordanite and tennantite in the same environment accounts for $\mathrm{Ag}$ and As being close to this group. The location of $\mathrm{S}$ and $\mathrm{Fe}$ in the low temperature group may be explained by the preferential sampling at the outer part of large spires. These samples are thus from the lowtemperature part of the chimneys and very often dominated by low-

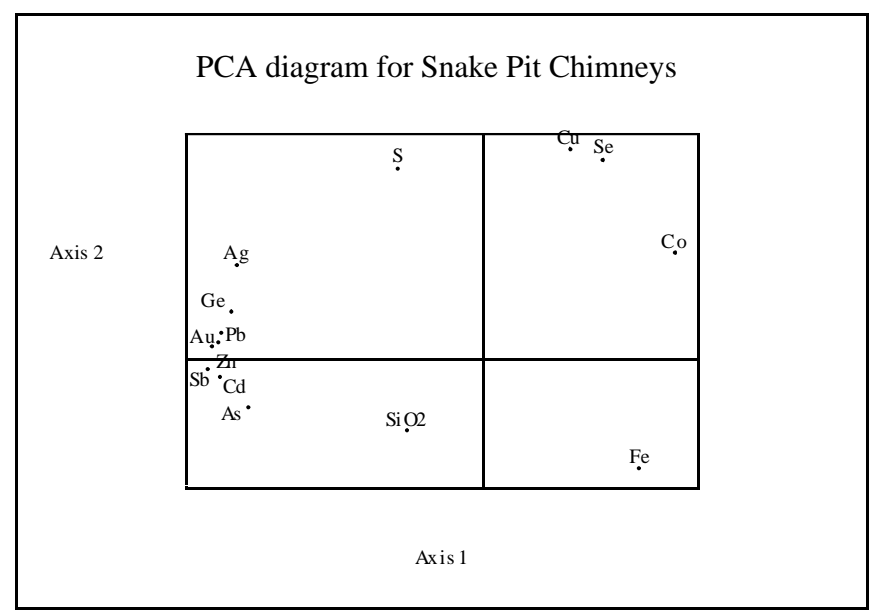

Percentage of the total variance expressed by each axis $\begin{array}{llll}\text { Axis } 157.1 \% & \text { Axis } 2 \quad 18.2 \% & \text { Axis } 3 \quad 10.4 \%\end{array}$

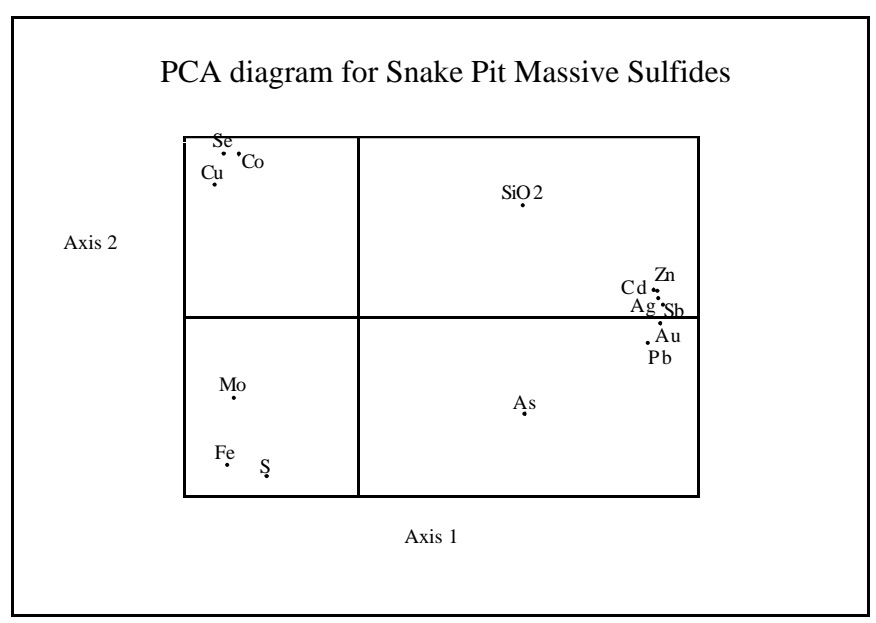

Percentage of the total variance expressed by each axis

$\begin{array}{llll}\text { Axis } 151.1 \% & \text { Axis } 2 & 27.4 \% & \text { Axis } 3 \quad 8.4 \%\end{array}$

Figure 12. Principal component analyses diagrams for Snakepit chimneys and massive sulfides.

temperature Fe-sulfides such as melnikovite, marcasite, and dendritic assemblages of a fine mixture of pyrite and marcasite. In the hightemperature group, $\mathrm{Cu}$ and $\mathrm{Se}$ are well correlated $(\mathrm{R}=0.80)$, but there is no correlation between these two elements and Co. The intermediate position of Co between $\mathrm{Cu}$ and $\mathrm{Fe}$ may indicate a Co concentration both in chalcopyrite and in pyrite, and most probably in hightemperature pyrite. The preferential location of Se in high-temperature black smokers has been observed at $13^{\circ} \mathrm{N}$ on the East Pacific Rise (Auclair et al., 1987)

Thus, for young, immature chimneys, the correlation between elements is well explained by the structure of chimneys and detailed mineralogical observations. Most of the minerals observed in the chimneys are growing relatively rapidly under unstable physicochemical conditions. In a chimney wall, the temperature gradient is very sharp and can drop from $350^{\circ} \mathrm{C}$ to a few degrees in a few centimeters. Thus, chimneys allow the study of the behavior of elements during a single stage under non-equilibrated physico-chemical conditions.

\section{Off-Axis Mature Deposits}

Off-axis deposits are Fe-rich massive sulfides. Deposits used for our calculation include mature samples from the top of the graben wall and the off-axial seamount (Fig. 13). These samples are up to 2 


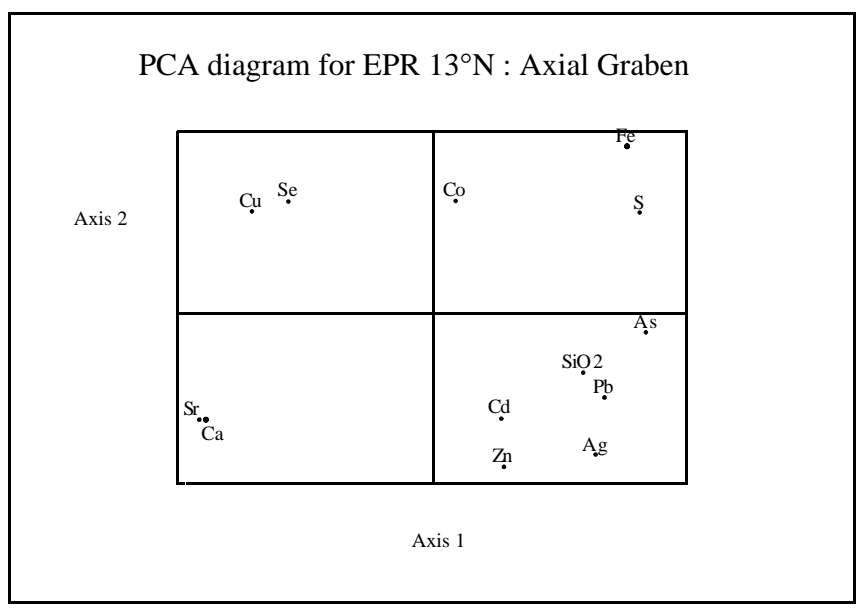

Percentage of the total variance expressed by each axis

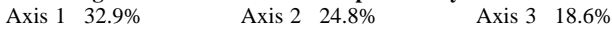

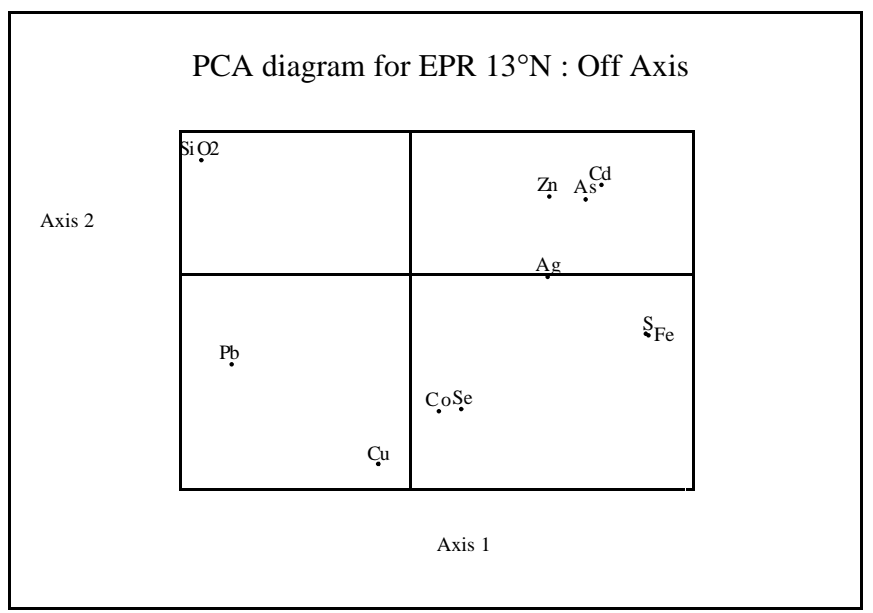

Percentage of the total variance expressed by each axis $\begin{array}{lllll}\text { Axis } 1 \quad 39.0 \% & \text { Axis } 2 & 25.0 \% & \text { Axis } 3 & 12.9 \%\end{array}$

Figure 13. Principal component analyses diagrams for EPR $13^{\circ} \mathrm{N}$ axial and off-axial deposits.

ka (Lalou et al., 1985). The samples are generally $\mathrm{Zn}$ poor, except one sample collected at the very top of a 70-m-high large mound. This sample corresponds to the last episode of activity. We may thus expect this sample to show enrichment of some elements because of zone refining within the mound. However, this sample is not particularly enriched in trace elements when compared to the axial Zn-rich chimney. Three explanations are possible: (1) there is no zone refining right now within the mound; (2) there is zone refining, but the offaxial deposits were never enriched in $\mathrm{Zn}$ and related elements; (3) there is zone refining, but most of the leached elements are dispersed in the ocean and few of them are deposited at the surface because of rapid venting and subsequent mixing in the open ocean.

Again, here axis 1 represents the temperature axis. $\mathrm{Se}$ and $\mathrm{Cu}$ are well correlated in the high-temperature part. $\mathrm{Ag}, \mathrm{Cd}$, and $\mathrm{Zn}$, and, to a lesser extent, $\mathrm{Pb}$ are associated in the low-temperature group. Arsenic is at an intermediate position along the temperature axis. This is different from axial samples, and may be explained by the preferential occurrence of As being substituted for Fe in pyrite rather than occurring in specific As minerals, as was the case in chimneys. Cobalt is close to As and is probably located in high-temperature pyrite. Silica is not correlated with any of the chalcophile elements. This position probably relates to the general occurrence of opal as a late mineral infilling the open spaces of the massive sulfides. This pervasive late silicification can affect any pre-existing sample of low-, medium-, or high-temperature, thus explaining the independence of silica relative to other elements. Compared to the chimneys, $\mathrm{Fe}$ is closer to the high temperature group. This can be explained by the more systematic occurrence of idiomorphic pyrite (i.e., high temperature) rather than the lower temperature, poorly crystallized, marcasite-pyrite assemblage observed in the chimneys. In mature sulfides, the correlation is better established for some elements than in the chimneys. This better correlation is related to maturation and refining processes.

\section{DISCUSSION}

In this part, we discuss the geochemical processes occurring within the mound for each element or group of elements and the behavior of elements in primary precipitates and during maturation processes. We also compare the TAG results with those from Snakepit and the East Pacific Rise $13^{\circ} \mathrm{N}$ because at both sites is a large mound of massive sulfides and samples were analyzed in our lab using the same techniques. Minor elements associated with major minerals (pyrite, chalcopyrite, and sphalerite) can be divided into three groups according to the most likely occurrence of these elements; as discussed in detail by Huston (Huston et al., 1995). As pyrite is by far the most common sulfide mineral, most of the above discussion, derived from bulk analyses, depends on trace-element behavior with respect to pyrite. In the first group are elements occurring mainly as mineral inclusions $(\mathrm{Cu}, \mathrm{Zn}, \mathrm{Pb}, \mathrm{Ba}, \mathrm{Bi}, \mathrm{Ag}$, and $\mathrm{Sb}) \mathrm{In}$ the second group are elements that occur as non stoichiometric substitutions in the lattice (As, $\mathrm{Tl}$, and Mo). In the third group are elements that occur as stoichiometric substitution for $\mathrm{Fe}(\mathrm{Co}$ and $\mathrm{Ni}$ ) or $\mathrm{S}$ ( $\mathrm{Se}, \mathrm{Te})$. In the following section we will discuss successively elements related to the gangue minerals $(\mathrm{Si}, \mathrm{Ca})$ then elements related to major sulfide minerals $(\mathrm{Cu}$, $\mathrm{Fe}, \mathrm{Zn}$ ), and finally trace elements.

\section{Silica}

Opal is dominant at the outer part of chimneys or as a late, generally minor phase cementing open spaces of older mineralogical assemblages. Quartz is enriched in the deepest parts of the mound, where part of the silica is derived from the basalt and was mobilized during the alteration process. The large amount of quartz at the center of the mound and the relative scarcity of opal at the surface can be explained by the silica solubility (Fig. 14) in Fournier (1983). According to this diagram the end-member hydrothermal fluid at TAG is close to the saturation for quartz (Edmond et al., 1995). Recent results for shallow vents also show a systematic equilibrium with quartz, indicating that silica tends to equilibrate in the fluid at near seabed pressure conditions.

The absence of quartz at the surface is because of the combination of kinetic effects and a preferential cooling of the end-member fluid by mixing with seawater. To oversaturate the fluid with respect to amorphous silica, the fluid has to be in part conductively cooled. As chimneys are direct and rapid pathways for the end-member fluid, there is generally a minimum of conductive cooling, and, thus, according to the silica solubility curves (Fig. 14), no possibility to precipitate amorphous silica. Silica chimneys are observed in some hydrothermal sites. The formation of these chimneys generally implies that the fluid is conductively cooled before venting on the seafloor (Herzig et al., 1988; Tivey and Delaney, 1986). In several cases, the precipitation of silica in this type of chimney is enhanced by filamentous bacterial activity (Juniper and Fouquet, 1988). During the evolution of the mound, the conductive cooling and restricted mixing of the end-member fluid probably forced the silica precipitation within the mound at approximately $20 \mathrm{~m}$. Amorphous silica can precipitate in the open spaces of the porous sulfides and seal the system creating an impermeable cap rock at the surface of the system. This is generally achieved at a low temperature. Once the fluid is trapped under 
this impermeable lid, conductive cooling is enhanced and mixing with ambient seawater is restricted, making the precipitation of opal more efficient. Such a system is not common in oceanic deposits and was not observed at Snakepit or at the East Pacific Rise $13^{\circ} \mathrm{N}$. The very hard layer containing red and gray chert encountered in the upper few meters of the cores recovered from the TAG-2, TAG-3, and TAG-4 areas was probably formed by such a process. Microscopic observation shows that opal is now being recrystallized into quartz. This layer may be analogous to the silica caps overlying many volcanogenic massive sulfides on land (Franklin et al., 1981). However, the Si content of white smoker fluids at TAG is just slightly lower $(19.1 \mathrm{mmol} / \mathrm{L})$ than for black smoker fluids $(20.75 \mathrm{mmol} / \mathrm{L})(\mathrm{Ed}-$ mond et al., 1995). This is not compatible with massive formation of opal. A possible explanation is that saturation is achieved very close to the surface of the mound and that the fluid is just starting to precipitate amorphous silica. This can explain the formation of a surficial silica cap (Fig. 15). The absence of abundant silica within the massive sulfides probably indicates that cooling by mixing is dominant over conductive cooling.

The abundance of quartz in the lower half of the mound is related to the saturation of the end-member fluid with respect to quartz. According to the solubility of quartz and the concentration of silica in the end-member fluid this implies a minimum of mixing with seawater and a slight conductive cooling. Thus, quartz must form at a temperature close to the end-member fluid temperature $\left(350^{\circ} \mathrm{C}\right)$. The systematic occurrence of quartz implies that a large part of the mound is at high-temperature. This internal high temperature, possibly enhanced by the near surface impermeable silica cap, agrees with systematic high temperature fluid inclusions measured in TAG quartz (Petersen et al., Chap. 13, this volume). Thus, stable and uniform physico-chemical conditions may explain the mineralogical and geochemical homogeneity of the sulfide minerals. As black smoker fluids are close to saturation in quartz, they cannot dissolve and transport over a long distance the silica derived from their reaction with

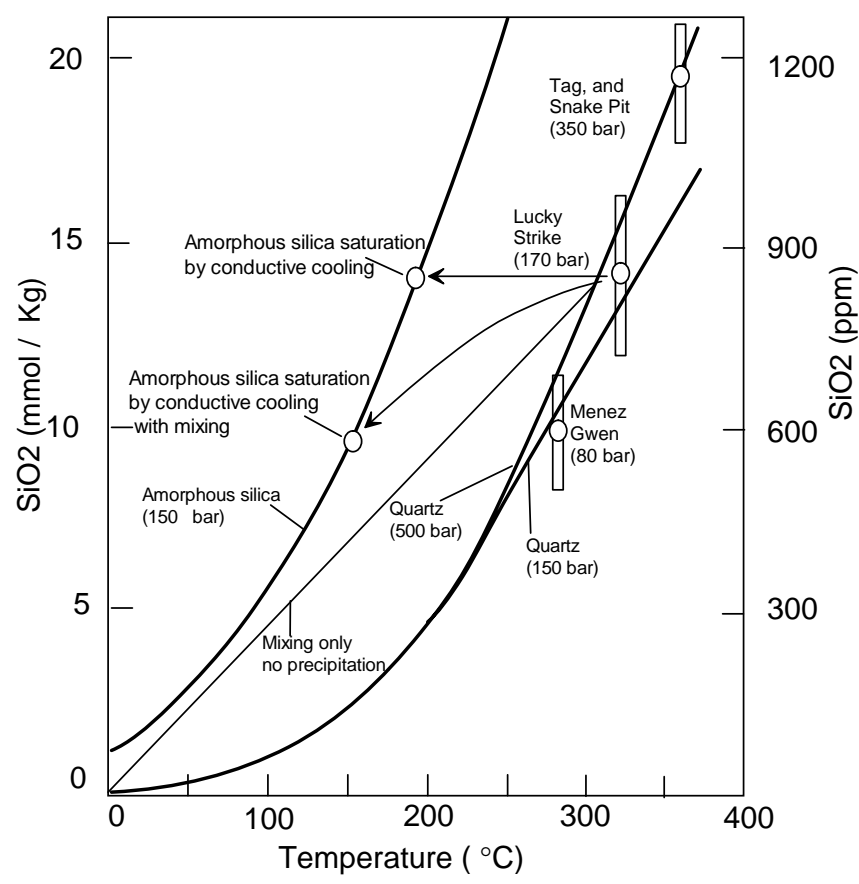

Figure 14. Solubility curve for amorphous silica and quartz (Fournier, 1983), with silica concentrations from hydrothermal sites at different depths. Si concentrations in the fluids from Lucky Strike, Menez Gwenn, and TAG are from J.L. Charlou (pers. comm, 1995). basalt. We thus consider that a large proportion of the quartz found at depth in the TAG mound is an in situ residue of basalt alteration.

PCA analyses, conducted on different types of TAG sulfides, demonstrate that silica is independent of other elements. This is compatible with both late pervasive cementation of preexisting mineral associations by silica and is also compatible with basalt alteration.

\section{Calcium and Strontium}

Calcium and strontium are strongly correlated. Anhydrite occurs either as thick veins or as breccia matrix cementing sulfide clasts of various types. The Black Smoker Complex itself is composed predominantly of massive anhydrite. Because of its retrograde solubility at high seafloor pressures and temperatures below $160^{\circ} \mathrm{C}$ (Blount and Dickson, 1969), anhydrite is unstable and dissolves when exposed to seawater. The abundance of anhydrite within the mound indicates either that ambient fluid temperatures are above the solubility limit of anhydrite, or that the anhydrite is isolated from continued exposure to circulating fluids of $<160^{\circ} \mathrm{C}$. The high-velocity discharge of hot fluid forces a major flow of seawater from the edges of the mound into the subsurface of the active Black Smoker Complex. Heating of this seawater to temperatures above $160^{\circ} \mathrm{C}$ and/or mixing with hightemperature fluid $\left(>360^{\circ} \mathrm{C}\right)$ may account for the abundance of anhydrite beneath the central Black Smoker Complex. We can speculate that the anhydrite-rich Black Smoker Complex is directly related to the anhydrite-rich zone of TAG-1. Two mechanisms have to be considered: mixing and heating. Conductive heating of seawater above $160^{\circ} \mathrm{C}$ will precipitate anhydrite within the mound; as this is a seawater-dominated process, we would not expect to have associated sulfides. Mixing between seawater and end-member fluid will precipitate anhydrite (Tivey et al., 1995) and high-temperature sulfides (chalcopyrite).

PCA diagrams show that $\mathrm{Ca}$ and $\mathrm{Sr}$ are generally independent of other elements at TAG. This probably indicates that veins occur within different types of preexisting material. In TAG-1 (Fig. 8) the location of $\mathrm{Cu}$ close to $\mathrm{Ca}$ indicates that an important part of the $\mathrm{Cu}$ is added to the inner mound as late chalcopyrite reoccurring at the anhydrite vein selvedges. At $13^{\circ} \mathrm{N}$ the association of $\mathrm{Ca}-\mathrm{Sr}$ and $\mathrm{Cu}-\mathrm{Se}$ along axis 1 characterizes the high-temperature association while their opposition along axis 2 indicates the difference between reducing (formation of sulfides) and oxidizing (formation of sulfates) conditions.

\section{Zinc}

Sphalerite is enriched at the surface of the deposits and particularly in the young immature (nonrecrystallized porous and generally poorly crystalline assemblages with typical primary chimney textures) deposits from TAG-4. This is demonstrated, for example, at $13^{\circ} \mathrm{N}$ on the East Pacific Rise where axial-immature deposits are enriched in $\mathrm{Zn}$ when compared to the mature $\mathrm{Zn}$-poor off-axial deposits. The same was observed in young chimneys from the Snakepit area when compared to the massive sulfides from the mound. Surface samples collected from TAG also indicate a $\mathrm{Zn}$-rich upper part, particularly in the Kremlin area. As the mound grows, collapsed Zn-rich chimneys are incorporated into the mound and contribute to a $\mathrm{Zn}$ enrichment. No Zn-rich zones are seen in the deep drill core at TAG, which implies a zone refining process occurring in the brecciated $\mathrm{Zn}$ rich material from the surface. Computed models indicate that reaction of high-temperature solutions with massive sulfide may significantly remobilize $\mathrm{Zn}$ within the massive sulfide deposit. Dissolution of anhydrite was demonstrated to be possible (Janecky and Shanks, 1988). Several observations of chimneys also show a systematic replacement of sphalerite by chalcopyrite at high temperature (Fouquet et al., 1988). At TAG, a particularly convincing argument is the significant $\mathrm{Zn}$ enrichment in the acid white smoker fluid $(400 \mu \mathrm{mol} / \mathrm{L})$ 


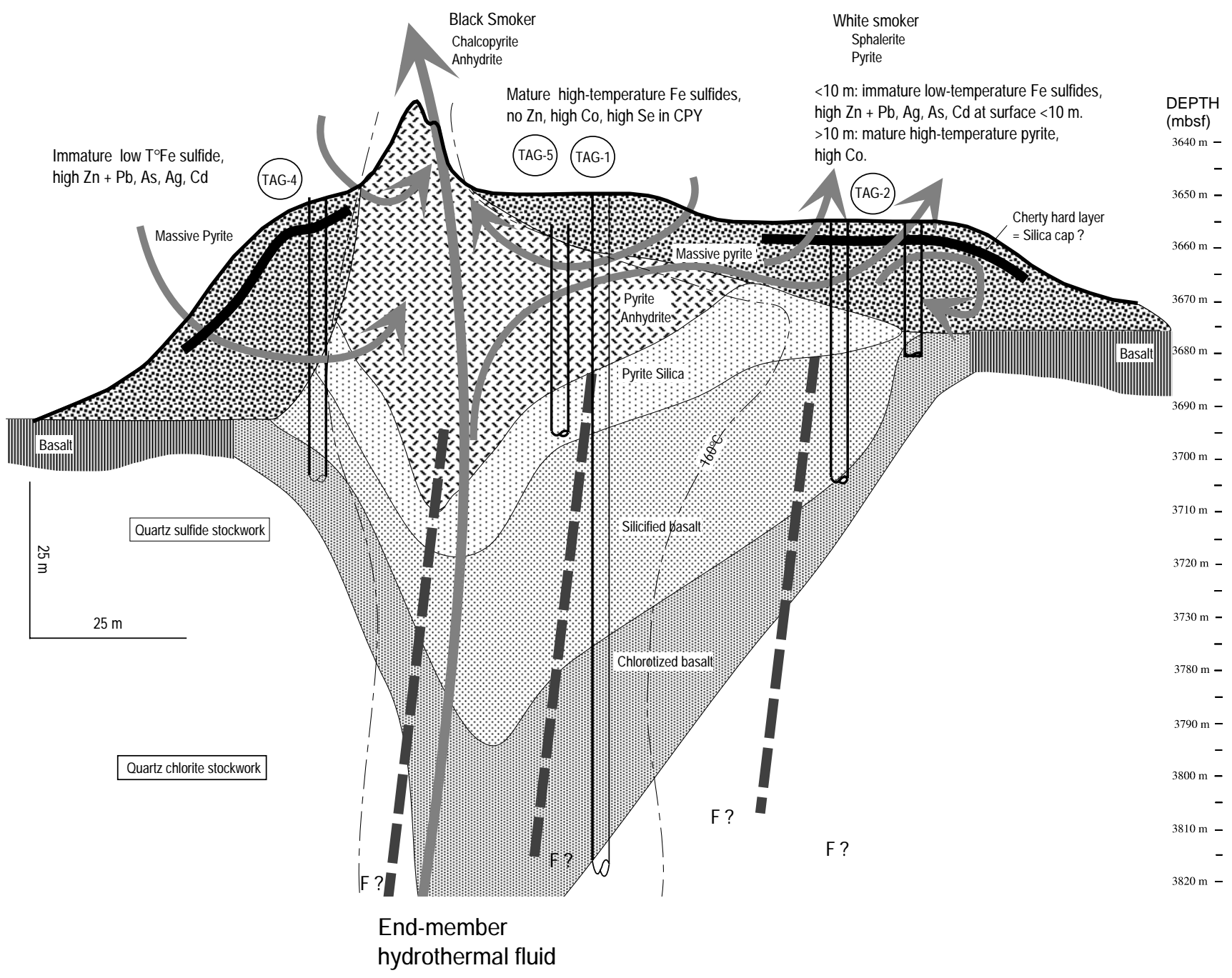

Figure 15. Schematic mineralogical and chemical zonation of the TAG mound. We believe that a Ca-Cu-rich zone exists under the Black Smoker Complex and is laterally connected to anhydrite veins drilled at TAG-1. The morphological, mineralogical, and geochemical asymmetry of the mound may be explained by ridge-parallel faults related to the rift valley wall system. Mineralogical and geochemical investigations suggest that cool seawater enters the mound at the western side (solid arrows) and near the top of the mound. The possible existence of a cherty impermeable layer may restrict mixing at the outer part of the mound. The limit of anhydrite occurrence (dashed line) is considered to define the limit of the $160^{\circ} \mathrm{C}$ isotherm within the mound. CPY $=$ chalcopyrite, $\mathrm{F}$ ? $=\mathrm{Possible}$ fault.

compared to the black smoker fluid (46 $\mu \mathrm{mol} / \mathrm{L}$; Edmond et al., 1995). Low $\mathrm{pH}$ because of extensive precipitation of pyrite within the mound causes large-scale mobilization of $\mathrm{Zn}$ and its precipitation at the surface or its expulsion with vent fluid. The high $\mathrm{Zn}$ content of the fluid means that much of the $\mathrm{Zn}$ is lost in the seawater column. This process contributes to the large-scale compositional zonation observed in Cyprus-type ore bodies (Franklin et al., 1981; Coleman et al., 1976). However, as the surface samples at TAG-1 are already mature and depleted in $\mathrm{Zn}$, it seems that this process is relatively rapid and efficient at least at the center of the mound. At TAG-4, most $\mathrm{Zn}$-rich samples are within the first $15 \mathrm{~m}$, but one high value in the deepest part indicates that the leaching process was incomplete in this section. This Zn-rich deep sample has a mineralogy (marcasite/pyrite) and textures (banded) typical of immature samples from the surface. The major difference is the size of marcasite and pyrite and late chalcopyrite. The immature porous pyrite-marcasite assemblage from the top of TAG- 4 can be considered as representative of the composition of massive sulfide before maturation and leaching of $\mathrm{ZnS}$. The end-member results of the leaching process are the mature Zn-poor massive sulfides at TAG-1. At TAG-2, the Zn-rich zone is within the first $15 \mathrm{~m}$. Thus, the zone of primary $\mathrm{Zn}$ enrichment is not very thick, and zone-refining processes for $\mathrm{Zn}$ can only be considered for the first $15 \mathrm{~m}$ of the mound. It is difficult to believe that the white smoker fluid can become enriched 10 times in $\mathrm{Zn}$ relative to black smoker parental fluid over such a short distance because, close to the surface, the fluids are mixed and thus diluted rapidly. The only possible way to increase the $\mathrm{Zn}$ content of the white smoker fluid would be to trap the fluid for a time. The existence of a superficial chert lid may be a good barrier for the fluid. However, a trapped fluid would cool conductively to precipitate pervasive amorphous silica, but this is not observed. Deeper in the system we may speculate that part of the $\mathrm{Zn}$ was leached during maturation; however, the presence of quartz and evidence for direct basalt replacement by quartz and pyrite in the quartz-rich part of the mound implies that this assemblage was 
formed in situ. Thus, the part of the deposit in contact with the hightemperature end-member fluid was never enriched in $\mathrm{Zn}$, and, therefore, was not affected by the refining process. According to the drilling results, the source of $\mathrm{Zn}$ in the white smoker fluid is not identified.

\section{Arsenic, Lead, Cadmium, and Silver}

These elements are the low-temperature group generally associated with, but not always directly correlated to, $\mathrm{Zn}$. Lead and $\mathrm{Cu}$ occur commonly as mineral inclusions of galena and chalcopyrite in sphalerite. Lattice substitution in sphalerite includes $\mathrm{Fe}, \mathrm{Cd}, \mathrm{Cu}, \mathrm{Ag}$, and possibly Mo and As. Sphalerite is very sensitive to dissolution by acid fluids, and elements trapped in the sphalerite lattice will be particularly mobile during this process. This raises the question of a possible concentration of these elements during maturation. At $13^{\circ} \mathrm{N}$ there is no significant difference in trace elements between young immature $\mathrm{Zn}$ chimneys at the axis and a $\mathrm{Zn}$ chimney growing at the top of the mound. The latter may be related to fluids that have leached $\mathrm{Zn}$ and associated elements from deeper in the system. Thus, some of these elements may be enriched in the fluid and dispersed in the seawater during venting without creating significant surface enrichment. In this case, the refining process seems not to be efficient to concentrate $\mathrm{As}, \mathrm{Pb}$, and $\mathrm{Ag}$ in the late chimneys at the top of the mound.

At TAG-4 the strong correlation between $\mathrm{Zn}, \mathrm{Cd}$, and As indicates that As probably occurs within the sphalerite lattice rather than in the pyrite. However, $\mathrm{Zn}$ was analyzed as a major element (detection limit $1000 \mathrm{ppm}$ ) and $\mathrm{Pb}, \mathrm{As}$ and $\mathrm{Ag}$ as minor elements. The relation between these elements and $\mathrm{Zn}$ cannot be discussed in detail. The absence of correlation between $\mathrm{Zn}$ and $\mathrm{Pb}-\mathrm{Ag}$ may indicate that these latter elements are preferentially located in the pyrite. Since the $\mathrm{Pb}$-As correlation coefficient is the same for TAG-1, -2 , and -4 , we conclude that there is a simultaneous increase or decrease of As and $\mathrm{Pb}$ both in sphalerite and pyrite. The dissolution of sphalerite will mobilize the As and lower the As concentration in the remaining mineralization as observed in the $\mathrm{Zn}$-poor mature samples. According to Huston (Huston et al., 1995) $\mathrm{Pb}$ and Ag occur preferentially as mineral inclusions in the pyrite and will be removed from the pyrite during recrystallization. This can be inferred from TAG-1 and TAG-4 where "high" $\mathrm{Pb}$ and Ag values in immature samples (TAG-4) are lowered during maturation (Table 1).

Some elements associated with Zn may be preferentially trapped in pyrite forming together with sphalerite. In this case there will be a good correlation with $\mathrm{Zn}$ in the immature samples and, when sphalerite dissolves, these elements may stay in the pyrite giving a poor correlation in mature samples. Enrichment of these elements ( $\mathrm{Pb}, \mathrm{As}$, $\mathrm{Cd}, \mathrm{Ag}$ ) could be an indicator about whether or not the sulfide minerals under consideration were formed at low temperature at the surface or were formed at high temperature within the mound. For example, As is enriched in surface samples at TAG-1 and its concentration then decreases and stabilizes at about $30 \mathrm{ppm}$ in the silicic part of the mound. The As enrichment within the sulfide part is better established at TAG-4 and TAG-2. The good correlation between As and $\mathrm{Zn}$ in TAG-4 $(\mathrm{R}=0.74)$ indicates a preferential association of As with low- to medium-temperature minerals. Detailed studies at $13^{\circ} \mathrm{N}$ showed that As (Auclair, 1988) was located in the pyrite lattice and that it often occurred at the low-temperature selvage of pyrite while Se and Co occurred in the high-temperature core (Auclair et al., 1987). Without sphalerite, the relative independence of $\mathrm{Fe}$ and $\mathrm{As}$ also indicates that As is preferentially concentrated in a specific type of pyrite. Huston et al. (1995) indicate that colloform pyrite grains related to rapid precipitation may incorporate much As. At $13^{\circ} \mathrm{N}$ there is no $\mathrm{Zn}$-As correlation in chimneys, which can be explained by the occurrence of jordanite at the outer part of chimneys. In the $13^{\circ} \mathrm{N}$ massive sulfides, As plots intermediate on PCA diagram between Fe and $\mathrm{Zn}$ group (Fig. 13) indicating a preferential location in low tem- perature pyrite. The same result is obtained for massive sulfide at Snakepit. To summarize, we propose that As is preferentially incorporated into the lattice of low-temperature pyrite formed together with sphalerite and then lost by leaching processes during the mound maturation and recrystallization of the pyrite or marcasite.

At Snakepit, $\mathrm{Pb}$ has a relatively good correlation with $\mathrm{As}$ as in TAG-1, 2 and $4(\mathrm{R}=0.72)$. We know that at Snakepit and at $13^{\circ} \mathrm{N}$, $\mathrm{Pb}$ occurs generally as tiny grains of galena or jordanite associated with sphalerite at the outer and cooler part of chimneys. The low $\mathrm{Pb}$ values in the mature, Zn-poor TAG samples imply that galena was probably leached during maturation processes. In the residual pyrite, $\mathrm{Pb}$ keeps its initial correlation to As.

\section{Molybdenum}

Mo is highly correlated with Fe in sulfides from TAG-1, 4, and 5 ( $\mathrm{R}=0.75,0.73$ and 0.80 ). In TAG-2 the Fe-Mo correlation is lower. The lack of correlation between $\mathrm{Cu}$ and $\mathrm{Zn}$ and Mo indicates that Mo occurs preferentially in pyrite. As there is no clear vertical variation, we may speculate that Mo is independent of depth, temperature, and also maturation processes. At Snakepit, Mo is not correlated to Fe in the massive sulfides and in the chimneys. The Mo behavior therefore is not particularly easy to discuss.

\section{Copper}

Copper is concentrated at the surface of the deposit in TAG-1 and in near-surface drill cuttings of TAG-2. This attests a contribution of black smoker chimneys to the growth of the mound. The second type of $\mathrm{Cu}$-enrichment is in the selvages of anhydrite veins within the mound. PCA analyses at TAG reveal a close relationship between $\mathrm{Cu}$ and $\mathrm{Ca}-\mathrm{Sr}$, indicating that most of the copper is vein related. Mineralogical observations show that chalcopyrite does not occur only as vein selvage, but also as a late mineral infilling the voids between other minerals. Thus, part of the copper is added to preexisting sulfides by the fluid circulating through the veins but is not important enough to show up in the analyses because few veins were used for our study. This type of vein occurs principally in TAG-1. The occurrence of chalcopyrite indicates that the veins were formed at temperatures higher than $300^{\circ} \mathrm{C}$. Thus, at the central part of the mound, fluid circulating in the veins is dominated by hydrothermal fluid rather than by seawater. In this case, the formation of anhydrite is caused by mixing rather than by simple conductive cooling of the fluid above $160^{\circ} \mathrm{C}$. However, according to the difference in copper content between the white smoker fluids $(3 \mu \mathrm{mol} / \mathrm{L})$ and black smoker fluids (120-150 $\mu \mathrm{mol} / \mathrm{L})$ (Edmond et al., 1995), we may expect the precipitation of a large amount of chalcopyrite within the mound. Such a $\mathrm{Cu}$ rich zone was not drilled. Either the zone does not exist, or it occurs as a vertical network of veins centered beneath the undrilled Black Smoker Complex. The observed $\mathrm{Cu}$ selvages at the borders of anhydrite veins may correspond to the lateral fringe of this zone. At Snakepit where the $\mathrm{Cu}$ concentration of the black smoker fluid (10$17 \mu \mathrm{mol} / \mathrm{L}$; Edmond et al., 1995), is lower than at TAG, a copper-rich zone was identified in the massive sulfide part and the stockwork zone where massive isocubanite forms thick veins. In addition, ODP drilling at Snakepit indicated that the first $7 \mathrm{~m}$ of the deposits was extremely enriched in $\mathrm{Cu}$ (Honnorez et al., 1990).,

The low $\mathrm{Cu}$ contents of the sulfides in the drill core at TAG imply that there has been little or no high-temperature venting through the talus pile at the edge of the mound.

\section{Cobalt and Selenium}

These are the only two trace elements showing a relative downward increase within the mound. The presence of Se and Co typically indicates high temperatures and end-member fluids. These two ele- 
ments thus may reflect a temperature increase within the mound. PCA analyses show that Co and Se are generally associated with the copper-rich high-temperature sulfides. In all four sites, Co and Se have a good correlation but are not associated with $\mathrm{Cu}$, which probably indicates that they do not occur in chalcopyrite, but in pyrite. Co is a typical element that has a stoichiometric substitution of Fe in pyrite. In surface samples collected by submersible, Se has a clear correlation with $\mathrm{Ca}$ and $\mathrm{Sr}$, indicating that it tends to be concentrated preferentially in chimneys, probably in chalcopyrite in equilibrium with the end-member black smoker fluid. This agrees with the high Se concentration in the chalcopyrite from a black smoker fragment at the top of the mound (TAG-1). Similarly, at Snakepit, Se is highly correlated to $\mathrm{Cu}$ in the chimney group, but the correlation is also high in the massive sulfide mound group. At Snakepit, massive sulfides from the core of the mound are extremely enriched in $\mathrm{Cu}$ when compared to samples from TAG. Selenium is principally enriched in isocubanite from the stockwork zone. In PCA plots of chimneys and mounds at Snakepit, Co is close to the $\mathrm{Cu}-\mathrm{Se}$ group. At $13^{\circ} \mathrm{N}$, the high $\mathrm{Cu}-\mathrm{Se}$ correlation is also present both in the axial chimneys and the off-axial massive sulfides. In both cases, Co is not correlated to the $\mathrm{Cu}$-Se group. Thus, it seems that there is no specific rule for Se and Co concentrations during maturation of mounds. More specifically, their behavior may depend on the amount of high-temperature chalcopyrite in equilibrium with the end-member fluid.

Selenium is only highly concentrated $(850 \mathrm{ppm})$ in a fragment of black smoker from the top of the TAG mound. This is close to the high Se values (up to $1000 \mathrm{ppm}$ ) from black smoker vents at $13^{\circ} \mathrm{N}$ on the East Pacific Rise (Auclair et al., 1987). At Snakepit, high Se values are also seen in the black smoker chimneys (up to $366 \mathrm{ppm}$ ) and in the isocubanite from the stockwork zone (up to $527 \mathrm{ppm}$; Fouquet et al., 1993b). High Se values are characteristic of chalcopyrite growing in contact with the unmixed end-member fluid.

At TAG, Co is enriched in massive pyrite. At $13^{\circ} \mathrm{N}, \mathrm{Co}$ is also enriched in massive pyrite from the off-axial mound. This might be because of preferential substitution of Co during reworking of sulfides. However, we consider that the temperature of formation of pyrite and the kinetics of pyrite growth are key factors in explaining this variation. We consider that Co is preferentially incorporated into hightemperature, idiomorphic pyrite, growing under stable conditions at the core of the mound, rather than in lower temperature pyrite related to chimneys. This would lead to the observed Co depletion in surface samples from TAG. Cobalt and Se occur as stoichiometric substitutions in idiomorphic pyrite stable under high temperature. These elements are not very sensitive to later dissolution and refining processes.

The apparent Se enrichment in TAG-5 sulfides and the association with trace pyrrhotite probably indicate a higher formation temperature. Because no chalcopyrite generation was found to be enriched in Se, it is likely that drilling did not intersect (or did not recover) samples from the feeders for the present black smoker.

Most ore from ancient deposits is mined from a zone of massive pyrite and chalcopyrite at the central part of the mound and within the stockwork. Several deposits contain several million tonnes of cupriferous stockwork. Similar stockwork-like mineralization is exposed by recent faulting in the Galapagos Rift (Embley et al., 1988), in the Lau Basin (Fouquet et al., 1993a) and at the Snakepit (Fouquet et al, 1993a) deposit. Localized small stockworks are exposed along the south East Pacific Rise (Renard et al., 1985). However, in comparison to stockwork deposits on land, these exposures are small and represent the uppermost part of the systems.

\section{CONCLUSIONS}

In the TAG mound, an asymmetry exists in the morphology and heat flow. In addition, breccia at the western part of the mound
(TAG-4) appears to be part of a talus pile rather than be from a stockwork zone (Shipboard Scientific Party, 1996). These features suggest the existence of a normal fault or series of fault steps beneath the mound (Figs. 2, 15). This is compatible with the ridge-parallel depression at the eastern side of the Black Smoker Complex (Kleinrock et al., 1996) and with the 15-20 m of displacement of basalt basement on opposite sides of the mound. The asymmetry is also noted in geochemical and mineralogical studies. The immature nature and the Zn-rich composition of the entire TAG-4 section, compared to the maturity and the virtual absence of $\mathrm{Zn}$ deeper than $10 \mathrm{~m}$ in the eastern holes, indicate a clear geochemical asymmetry of the mound. The Black Smoker Complex is fed by a ridge-parallel fault that controls the thermal, morphological, and geochemical asymmetry of the mound. The anhydrite-rich zone is probably connected to the Black Smoker Complex. These results are summarized in the interpretative section presented in Figure 15.

TAG-4 is less affected by leaching of trace elements and is less mature than the other holes. TAG- 1 is completely depleted in trace metals, and TAG-2 is depleted in base metals deeper than $10 \mathrm{~m}$. This indicates that refining processes only occur in the first $10 \mathrm{~m}$ in the eastern mound. In addition, trace elements occurring in sphalerite are preferentially leached during maturation processes. Elements mobilized by refining are probably more concentrated in the $\mathrm{Zn}$ chimneys at the surface of the mound, these samples were not collected by drilling. The only enriched samples are probably the $\mathrm{Zn}$ chimneys from the surface indicating that the potentially enriched zone has virtually no thickness. Data obtained at $13^{\circ} \mathrm{N}$ on the East Pacific Rise indicate that $\mathrm{Zn}$ chimneys from the top of a mound are not specifically enriched in trace metals when compared with young chimneys growing directly on basalt. Thus, the fluid enriched in trace metals by a leaching process may only precipitate a minor part of these elements, most of which are dispersed into the seawater column.

In relation to these different observations, we consider that the mound growth is due to three different processes: (1) At the surface, the sulfides formed within the chimneys are progressively buried and recrystallized, contributing to the surface growth of the massive sulfide mound. This material can be relatively enriched in $\mathrm{Cu}$ and $\mathrm{Zn}$, which is later leached through a zone-refining process. (2) In the deepest part, the basalt is progressively silicified and replaced by a pyrite/quartz assemblage contributing to the internal growth and stockwork formation. These samples correspond to high-temperature pyrite and were never enriched in $\mathrm{Zn}$ and associated trace metals. Thus, most of the mound is depleted in trace metals and cannot be considered as a source for the high $\mathrm{Zn}$ of the white smoker fluids. The third process is the anhydrite veining that also contributes to the internal growth. Most of the anhydrite should be dissolved when the activity ceases. However veins are often associated with a chalcopyrite selvage that contributes to the inner mound growth.

No $\mathrm{Cu}$-rich zone was identified during drilling. The extremely high value of $\mathrm{Cu}$ in the end-member fluid from TAG when compared to Snakepit suggests that a vertical $\mathrm{Cu}$-rich zone exists under the Black Smoker Complex. $\mathrm{Cu}$ enrichment on vein selvages drilled at TAG- 1 could be considered as the external expression of the fringes of this zone.

\section{ACKNOWLEDGMENTS}

This paper has benefited from the careful reviews of B. Nesbitt, R. Binns, P. Herzig, and the ODP editorial comments.

\section{REFERENCES}

Alabaster, T., Pearce, J.A., Mallick, D.I.J., and Elboushi, I.M, 1980. The volcanic stratigraphy and location of massive sulphide deposits in the Oman Ophiolite. In Panayiotou, A. (Ed.), Ophiolites, Proceedings of the Inter- 
national Ophiolite Symposium. Geol. Surv. Dep., Nicosia, Cyprus, 751757.

Auclair, G., 1988. Processus de métallogenèse dans les amas sulfurés océaniques [Thèse Doctorat]. Univ. de Bretagne Occidentale, Brest.

Auclair, G., Fouquet, Y., and Bohn, M., 1987. Distribution of selenium in high-temperature hydrothermal sulfide deposits at $13^{\circ} \mathrm{N}$, East Pacific Rise. Can. Mineral., 25:577-588.

Becker, K., and Von Herzen, R.P., 1996. Pre-drilling observations of conductive heat flow at the TAG active mound using Alvin. In Humphris, S.E., Herzig, P.M., Miller, D.J., et al., Proc. ODP, Init. Repts., 158: College Station, TX (Ocean Drilling Program), 23-29.

Becker, K., Von Herzen, R.P., and Rona, P.A., 1993. Conductive heat flow measurements using Alvin at the TAG active hydrothermal mound, MAR at $26^{\circ}$ N. Eos, 74 (Suppl.): 99.

Beyer, W.H., 1968. Handbook of Tables for Probability and Statistics: Boca Raton, Fl (CRC Press).

Bideau, D., Fouquet, Y., and Hékinian, R., 1985. Découverte de basaltes métamorphisés dans le graben axial de la Dorsale Est Pacifique à $12^{\circ} 50$ 'N. Bull. Soc. Geol. Fr., 1:905-913.

Blount, C.N., and Dickson, F.W., 1969. The solubility of anhydrite $\left(\mathrm{CaSO}_{4}\right)$ in $\mathrm{NaCl}-\mathrm{H}_{2} \mathrm{O}$ from 100 to $450^{\circ} \mathrm{C}$ and 1 to 100 bars. Geochim. Cosmochim. Acta, 33:227-245.

Coleman, R.G., Houston, C.C., El-Boushi, I.M., Al-Hinai, K.M., and Bailey, E.B., 1976. The Semail ophiolite and associated massive sulfide deposits, Sultanate of Oman. In Tahoun, S.A. (Ed.), Evolution and Mineralization of the Arabian-Nubian Shield: New York (Pergamon Press), 179-192.

Constantinou, G., and Govett, G.J.S., 1973. Geology, geochemistry, and genesis of Cyprus sulfide deposits. Econ. Geol., 68:843-858.

Edmond, J.M., Campbell, A.C., Palmer, M.R., German, C.R., Klinkhammer, G.P., Edmonds, H.N., Elderfield, H., Thompson, G., and Rona, P., 1995. Time series studies of vent fluids from the TAG and MARK sites (1986, 1990): Mid-Atlantic Ridge: a new solution chemistry model and a mechanism for $\mathrm{Cu} / \mathrm{Zn}$ zonation in massive sulfide ore bodies. In Parson, L.M., Walker, C.L., and Dixon, D.R. (Eds.), Hydrothermal Vents and Processes. Geol. Soc. Spec. Publ. London, 87:77-86.

Embley, R.W., Jonasson, I.R., Perfit, M.R., Franklin, J.M., Tivey, M.A., Malahoff, A., Smith, M.F., and Francis, T.J.G., 1988. Submersible investigation of an extinct hydrothermal system on the Galapagos Ridge: sulfide mounds, stockwork zone, and differentiated lavas. Can. Mineral., 26:517-539.

Fouquet, Y., Auclair, G., Cambon, P., and Etoubleau, J., 1988. Geological setting and mineralogical and geochemical investigations on sulfide deposits near $13^{\circ} \mathrm{N}$ on the East Pacific Rise. Mar. Geol., 84:143-178.

Fouquet, Y., Knott, R., Cambon, P., Fallick, A., Rickard, D., and Desbruyeres, D., 1996. Formation of large sulfide mineral deposits along fast spreading ridges: example from off axial deposits at $12^{\circ} 43^{\prime} \mathrm{N}$ on the East Pacific Rise. Earth Planet. Sci. Lett., 144:147-162.

Fouquet, Y., von Stackelberg, U., Charlou, J.L., Erzinger, J., Herzig, P.M., Mühe, R., and Wiedicke, M., 1993a. Metallogenesis in back-arc environments: the Lau Basin example. Econ. Geol., 88:2154-2181.

Fouquet, Y., Wafik, A., Cambon, P., Mevel, C., Meyer, G., and Gente, P., 1993b. Tectonic setting and mineralogical and geochemical zonation in the Snakepit sulphide deposit (Mid-Atlantic Ridge at $23^{\circ} \mathrm{N}$ ). Econ. Geol., 88:2018-2036.

Fournier, R.O., 1983. A method of calculating quartz solubilities in aqueous sodium chloride solutions. Geochim. Cosmochim. Acta, 47:579-586.

Franklin, J.M., Lyndon, J.W., and Sangster, D.F., 1981. Volcanic-associated massive sulfide deposits. Econ. Geol., 75th Anniv. Vol., 75:485-627.

Hannington, M.D., Thompson, G., Rona, P.A., and Scott, S.D., 1988. Gold and native copper in supergene sulfides from the Mid-Atlantic Ridge. Nature, 333:64-66.

Hannington, M.D., Tivey, M.K., Larocque, A.C.L., Petersen, S., and Rona, P.A., 1995. The occurrence of gold in sulfide deposits of the TAG hydrothermal field, Mid-Atlantic Ridge. Can. Mineral., 33:1285-1310.

Herzig, P.M., Becker, K.P., Stoffers, P., Backer, H., and Blum, N., 1988. Hydrothermal silica chimney fields in the Galapagos spreading center at $86^{\circ}$ W. Earth Planet. Sci. Lett., 89:261-272.

Herzig, P.M., Hannington, M.D., Scott, S.D., Maliotis, G., Rona, P.A., and Thompson, G., 1991. Gold-rich seafloor gossans in the Troodos ophiolite and on the Mid-Atlantic Ridge. Econ. Geol., 86:1747-1755.

Honnorez, J., Mevel, C., and Honnorez-Guerstein, B.M., 1990. Mineralogy and chemistry of sulfide deposits drilled from hydrothermal mound of the
Snake Pit active field, MAR. In Detrick, R., Honnorez, J., Bryan, W.B., Juteau, T., et al., Proc. ODP, Sci. Results, 106/109: College Station, TX (Ocean Drilling Program), 145-162.

Humphris, S.E., Herzig, P.M., Miller, D.J., Alt, J.C., Becker, K., Brown, D., Brügmann, G., Chiba, H., Fouquet, Y., Gemmell, J.B., Guerin, G., Hannington, M.D., Holm, N.G., Honnorez, J.J., Itturino, G.J., Knott, R., Ludwig, R., Nakamura, K., Petersen, S., Reysenbach, A.-L., Rona, P.A., Smith, S., Sturz, A.A., Tivey, M.K., and Zhao, X., 1995. The internal structure of an active sea-floor massive sulphide deposit. Nature, 377:713-716.

Humphris, S.E., Kleinrock, M.C., and the Deep-TAG Team, 1994. Detailed morphology and the distribution of venting at the active TAG hydrothermal mound, $26^{\circ} \mathrm{N}$, Mid-Atlantic Ridge. Eos, 75:660.

Huston, D.L., Sie, S.H., Suter, G.F., Cooke, D.R., and Both R.A., 1995. Trace elements in sulfide minerals from Eastern Australia volcanic-hosted massive sulfide deposits: Part I. Proton microprobe analyses of pyrite, chalcopyrite and sphalerite, and Part II. Selenium levels in pyrite: Comparisons with $\delta^{34} \mathrm{~S}$ values and implications for the source of sulfur in volcanogenic hydrothermal systems. Econ. Geol., 90:1167-1196.

Ixer, R.A., Alabaster, T., and Pearce, J.A., 1984. Ore petrography and geochemistry of massive sulphide deposits within the Semail Ophiolite, Oman. Trans. Min. Metall., Sect. B, 93:B114-B124.

Janecky, D.R., and Shanks, W.C., III, 1988. Computational modeling of chemical and sulfur isotopic reaction processes in seafloor hydrothermal systems: chimneys, massive sulfides, and subjacent alteration zones. Can. Mineral., 26:805-825.

Juniper, S.K., and Fouquet, Y., 1988. Filamentous iron-silica deposits from modern and ancient hydrothermal sites. Can. Mineral., 26:859-869.

Kleinrock, M.C., Humphris, S.E., and the Deep-TAG Team, 1996. Detailed structure and morphology of the TAG active hydrothermal mound and its geotectonic environment. In Humphris, S.E., Herzig, P.M., Miller, D.J., et al., Proc. ODP, Init. Repts., 158: College Station, TX (Ocean Drilling Program), 15-21.

Lalou, C., Brichet, E., and Hekinian, R., 1985. Age dating of sulfide deposits from axial and off-axial structures on the East Pacific near $12^{\circ} 50^{\prime} \mathrm{N}$. Earth Planet. Sci. Lett., 75:59-71.

Lalou, C., Brichet, E., and Reyss, J.L., 1993. Chronology of recently discovered hydrothermal mounds in the TAG area. Eos, 74:100.

Lalou, C., Thompson, G., Arnold, M., Brichet, E., Druffel, E., and Rona, P.A., 1990. Geochronology of TAG and Snakepit hydrothermal fields, Mid-Atlantic Ridge: witness to a long and complex hydrothermal history. Earth Planet. Sci. Lett., 97:113-128.

Purdy, G.M., Sempéré, J.C., and Schouten, H., 1990. Bathymetry of the MidAtlantic Ridge, $24^{\circ}-31^{\circ} \mathrm{N}$ : a map series. Mar. Geophys. Res., 12:247252.

Renard, V., Hekinian, R., Francheteau, J., Ballard, R.D., and Backer, H., 1985. Submersible observations at the axis of the ultra-fast-spreading East Pacific Rise $\left(17^{\circ} 30^{\prime}\right.$ to $21^{\circ} 30^{\prime}$ S). Earth Planet. Sci. Lett., 75:339353.

Rona, P.A., Bogdanov, Y.A., Gurvich, E.G., Rimski-Kursakov, A., Sagalevitch, A.M., Hannington, M.D., and Thompson, G., 1993a. Relict hydrothermal zones in the TAG hydrothermal field, Mid-Atlantic Ridge $26^{\circ} \mathrm{N}$, $45^{\circ}$ W. J. Geophys. Res., 98:9715-9730.

Rona, P.A., Hannington, M.D., Raman, C.V., Thompson, G., Tivey, M.K., Humphris, S.E., Lalou, C., and Petersen, S., 1993b. Active and relict seafloor hydrothermal mineralization at the TAG hydrothermal field, MidAtlantic Ridge. Econ. Geol., 88:1987-2013.

Rona, P.A., Klinkhammer, G., Nelson, T.A., Trefry, J.H., and Elderfield, H., 1986. Black smokers, massive sulfides and vent biota on the Mid-Atlantic Ridge. Nature, 321:33-37.

Rona, P.A., Thompson, G., Mottl, M.J., Karson, J.A., Jenkins, W.J., Graham, D., Mallette, M., Von Damm, K., and Edmond, J.M., 1984. Hydrothermal activity at the TAG hydrothermal field, Mid-Atlantic Ridge crest at $26^{\circ} \mathrm{N}$. J. Geophys. Res., 89:11365-11377.

Sempéré, J.-C., Purdy, G.M., and Schouten, H., 1990. Segmentation of the Mid-Atlantic Ridge between $24^{\circ} \mathrm{N}$ and $30^{\circ} 40^{\prime} \mathrm{N}$. Nature, 344:427-431.

Shipboard Scientific Party, 1996. Introduction and principal results. In Humphris, S., Herzig, P, and Miller, P. (Eds.), Proc. ODP, Init. Repts., 158: College Station, TX (Ocean Drilling Program), 5-14.

Smith, D.K., and Cann, J.R., 1992. The role of seamount volcanism in crustal construction at the Mid-Atlantic Ridge $\left(24^{\circ}-30^{\circ} \mathrm{N}\right)$. J. Geophys. Res., 97:1645-1658. 
Temple, D.G., Scott, R.B., and Rona, P.A., 1979. Geology of a submarine hydrothermal field, Mid-Atlantic Ridge, $26^{\circ} \mathrm{N}$ latitude. J. Geophys. Res., 84:7453-7466.

Thompson, G., Bryan, W.B., Ballard, R., Hamuro, K., and Melson, W.G., 1985. Morphology, mineralogy, and chemistry of hydrothermal deposits from the TAG area, $26^{\circ} \mathrm{N}$ Mid-Atlantic Ridge. Chem. Geol., 49:243-257.

Thompson, G., Humphris, S.E., Schroeder, B., Sulanowska, M., and Rona, P.A., 1988. Active vents and massive sulfides at $26^{\circ} \mathrm{N}$ (TAG) and $23^{\circ} \mathrm{N}$ (Snakepit) on the Mid-Atlantic Ridge. Can. Mineral., 26:697-711.

Tivey, M.K., and Delaney, J.R., 1986. Growth of large sulfide structures on the Endeavour Segment of the Juan de Fuca Ridge. Earth Planet. Sci. Lett., 77:303-317.
Tivey, M.K., Humphris, S.E., Thompson, G., Hannington, M.D., and Rona, P.A., 1995. Deducing patterns of fluid flow and mixing within the TAG active hydrothermal mound using mineralogical and geochemical data. $J$. Geophys. Res., 100:12527-12555.

Date of initial receipt: 6 June 1996

Date of acceptance: 11 April 1997

Ms 158SR-216 